\title{
Fluoromethyl-2,4,6-trinitrophenylsulfonate: A new
}

electrophilic monofluoromethylating reagent

Marco Reichel, ${ }^{\dagger}$ Sami Sile,${ }^{\dagger \dagger}$ Andreas Kornath, ${ }^{\dagger}$ Konstantin Karaghiosoff ${ }^{*} *$

$\dagger$ Department of Chemistry, Ludwig-Maximilian University Munich, Butenandtstr. 5-13 (D), 81377 Munich

$\dagger \dagger$ School of Natural and Environmental Sciences, Agriculture Building, King's Road, Newcastle upon Tyne NE1 7RU

*klk@.cup.uni-muenchen.de 
Inhalt

1 NMR Spectra

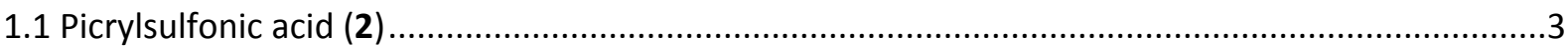

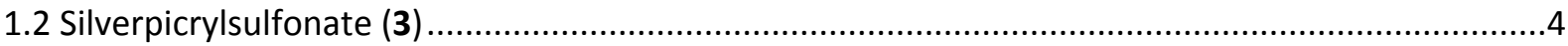

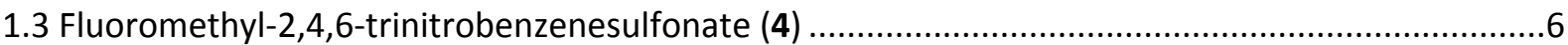

1.4 2-(Fluoromethyl)isouronium-2,4,6-trinitrobenzenesulfonate (5a) ..........................................8

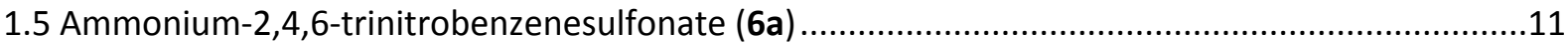

1.6 Dimethylacetamidiniumfluoromethyl-2,4,6-trinitrobenzenesulfonate (5b) ...............................12

1.7 Dimethylformamidiniumfluoromethyl-2,4,6-trinitrobenzenesulfonate $(\mathbf{5 c})$..............................14

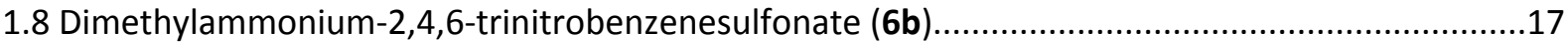

1.9 Dimethylammonium- $\mathrm{d}_{2}-2,4,6$-trinitrobenzenesulfonate $(\mathbf{6 b})$.................................................18

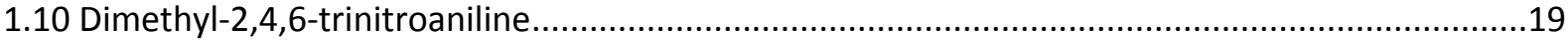

1.7 Fluoromethylthiotriphenylphosphonium-2,4,6-trinitrobenzenesulfonate (7b) ...........................20

1.12 Fluoromethylselenotriphenylphosphonium-2,4,6-trinitrobenzenesulfonate (7c) ......................23

1.13 4-(4-(Dimethylamino) benzoyl) - $\mathrm{N}$ - (fluoromethyl) - N,N - dimethyl benzen aminium -2,4,6-

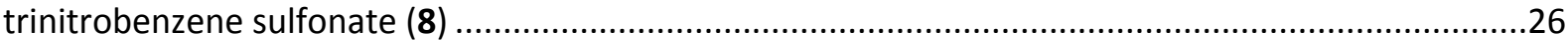

1.14 1-(Fluoromethyl)-2-picolinoylpyridin-1-ium 2,4,6-trinitrobenzenesulfonate (9) ........................29

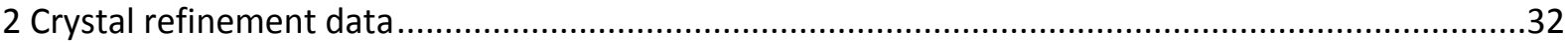

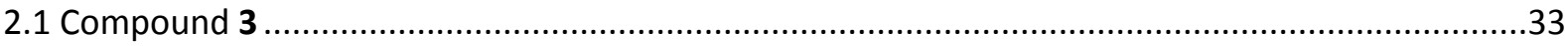

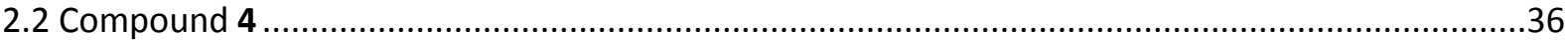

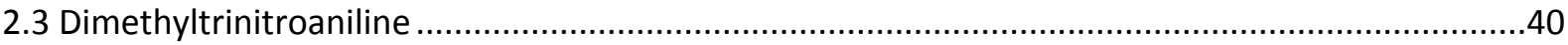

2.4 Compound 5a

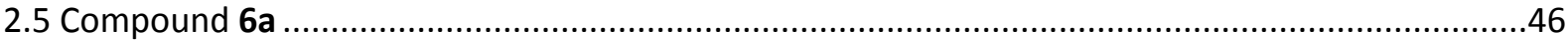

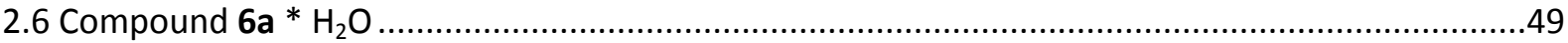

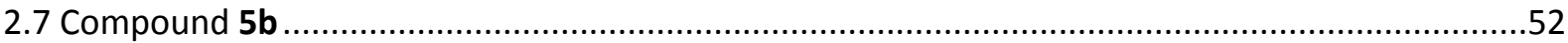

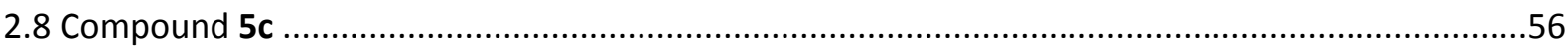

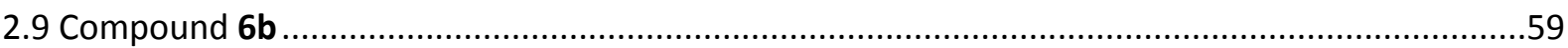

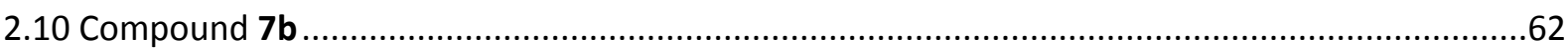

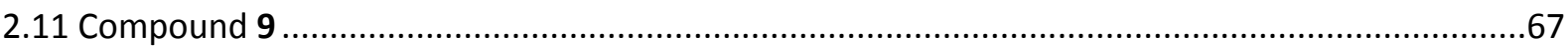

3 DSC of compound 4 


\section{NMR Spectra}

1.1 Picrylsulfonic acid (2)
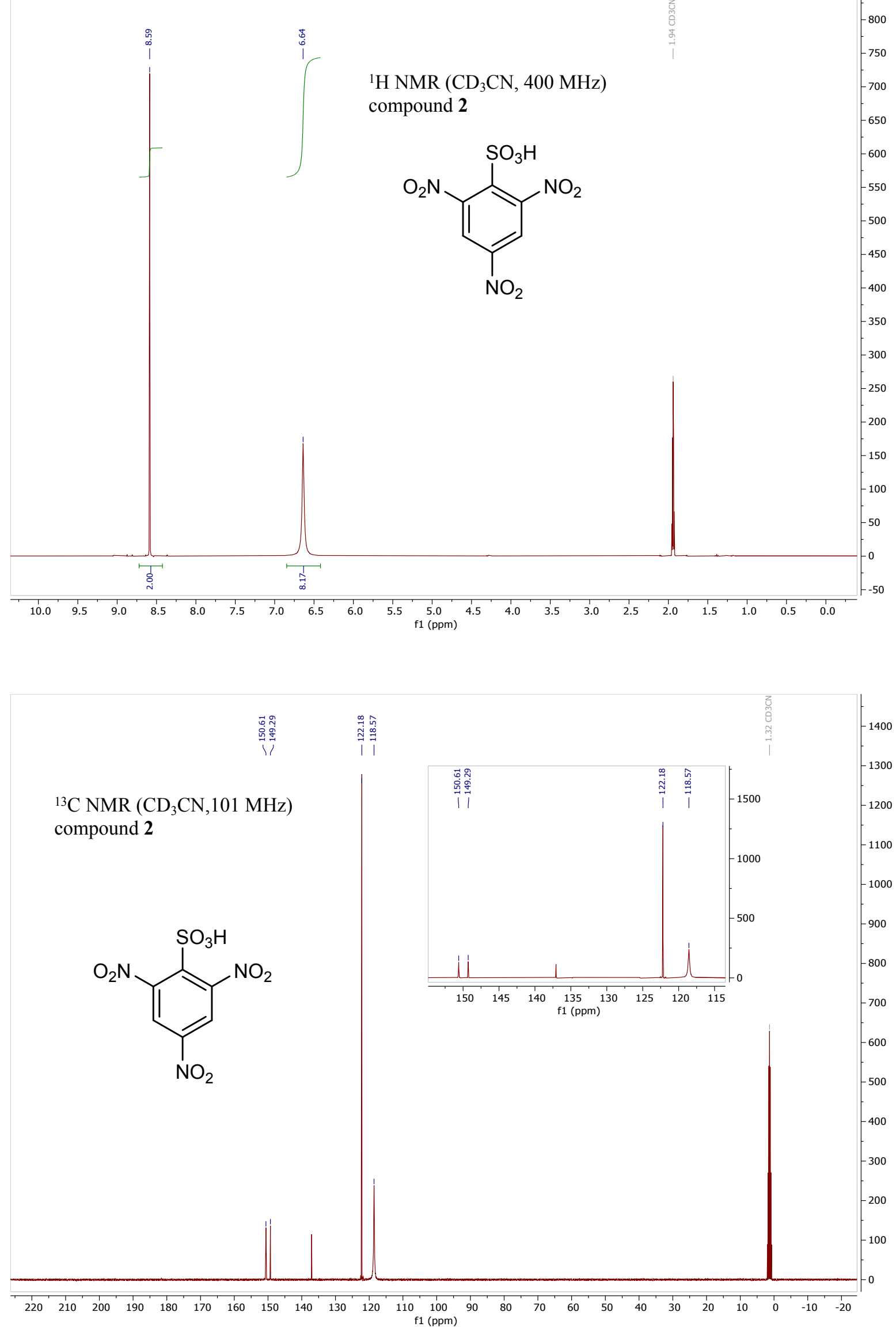


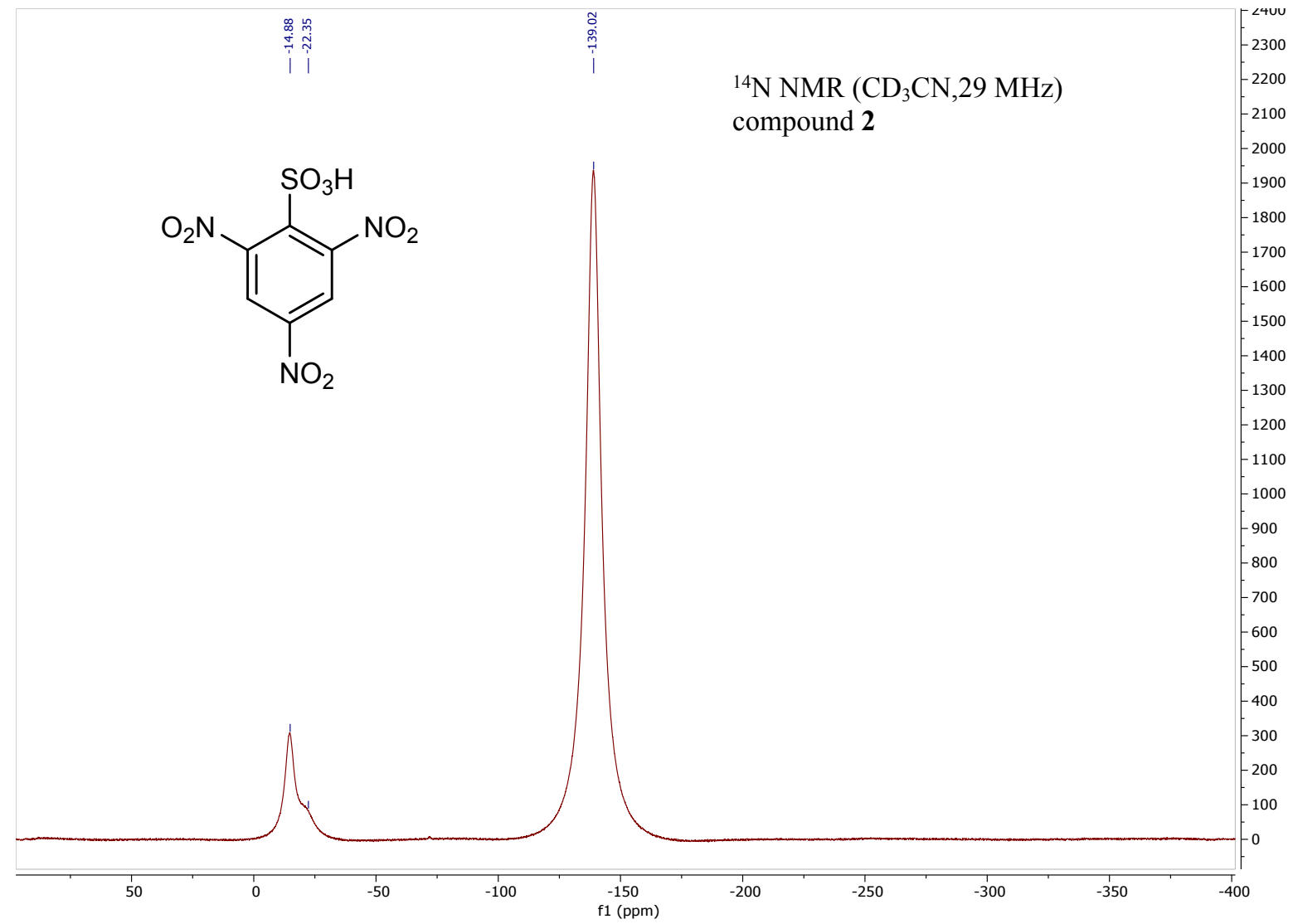

1.2 Silverpicrylsulfonate (3)

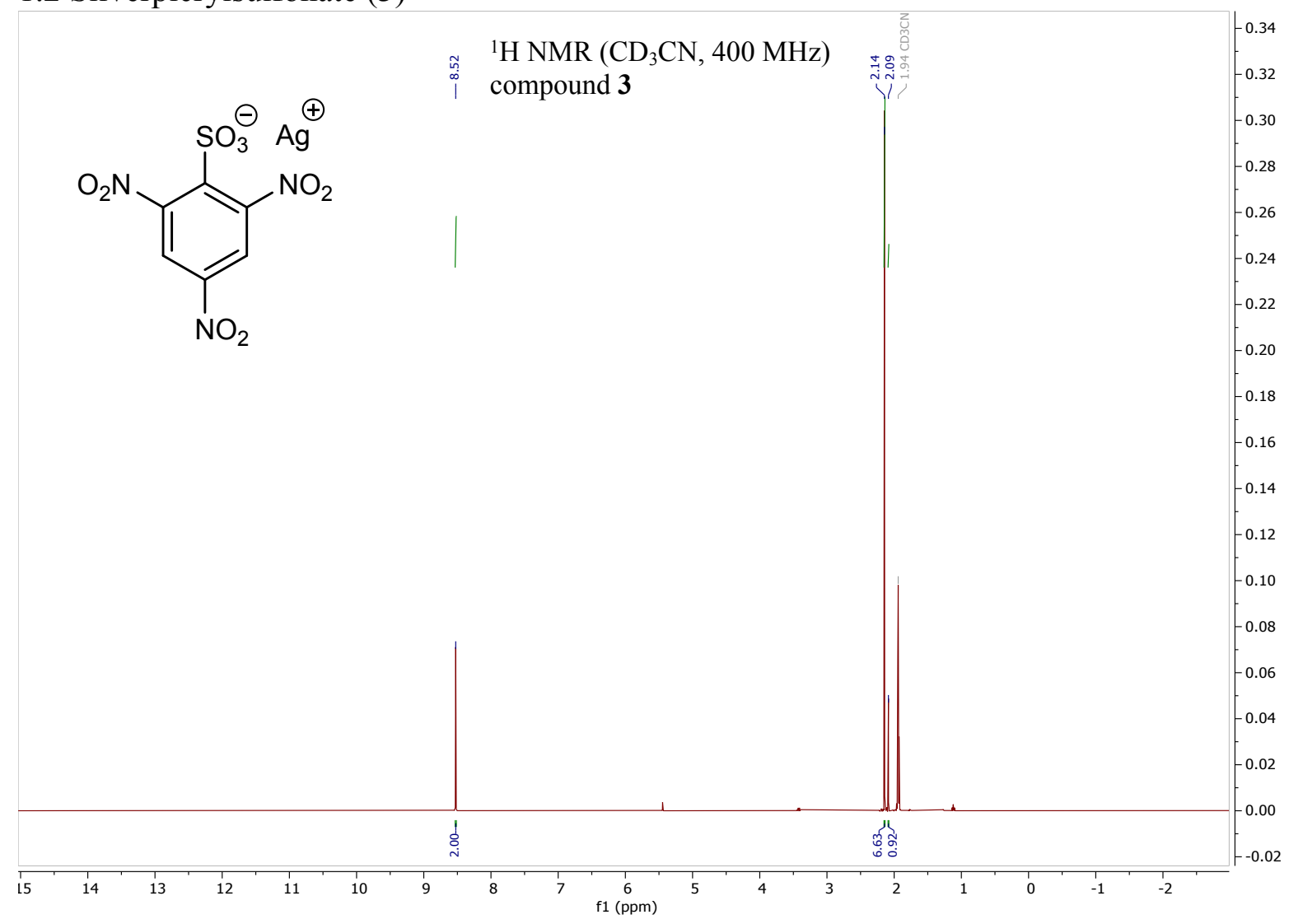



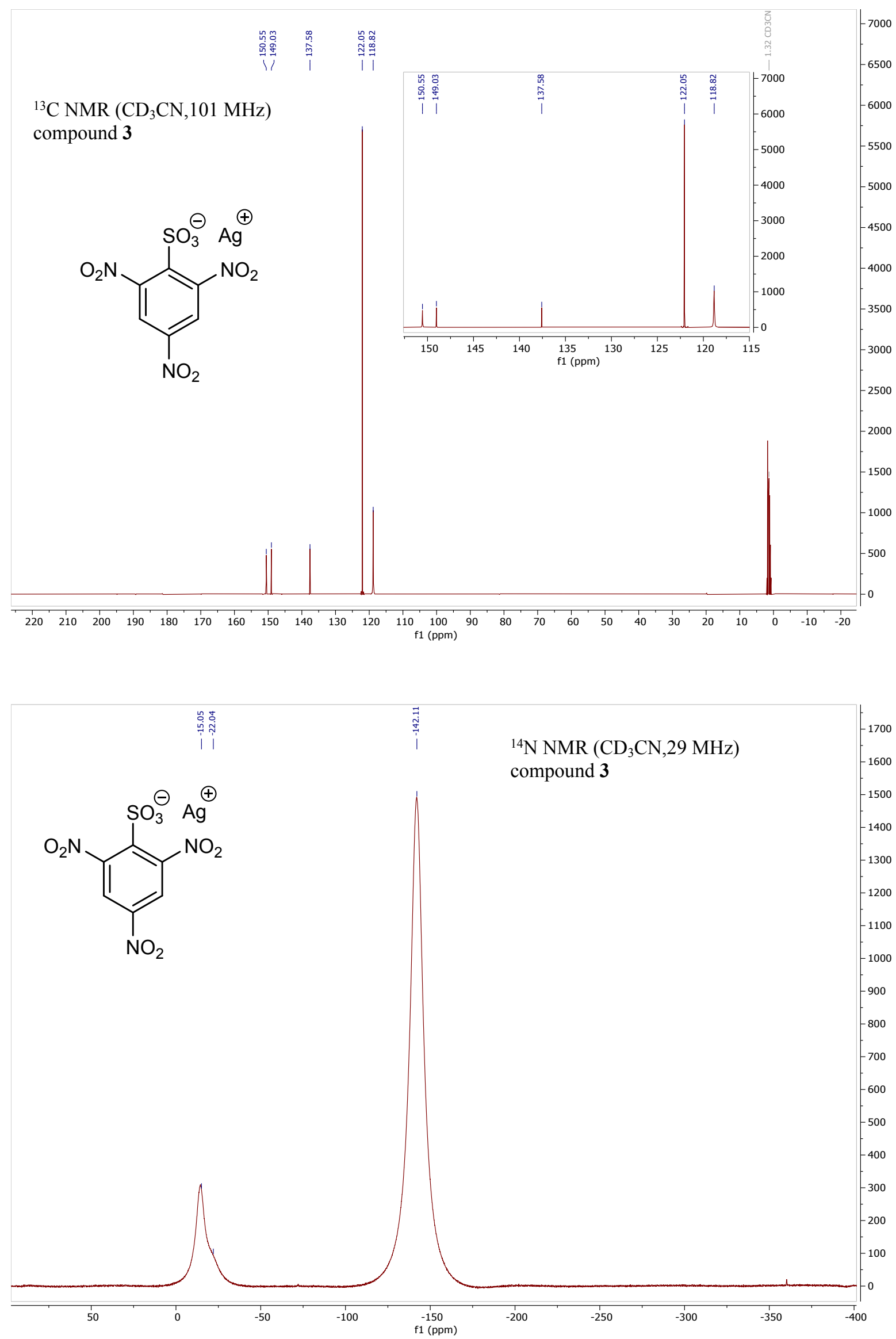
1.3 Fluoromethyl-2,4,6-trinitrobenzenesulfonate (4)

${ }^{1} \mathrm{H}$ NMR (d6-acetone, 400

$\mathrm{MHz}$ ), compound 4
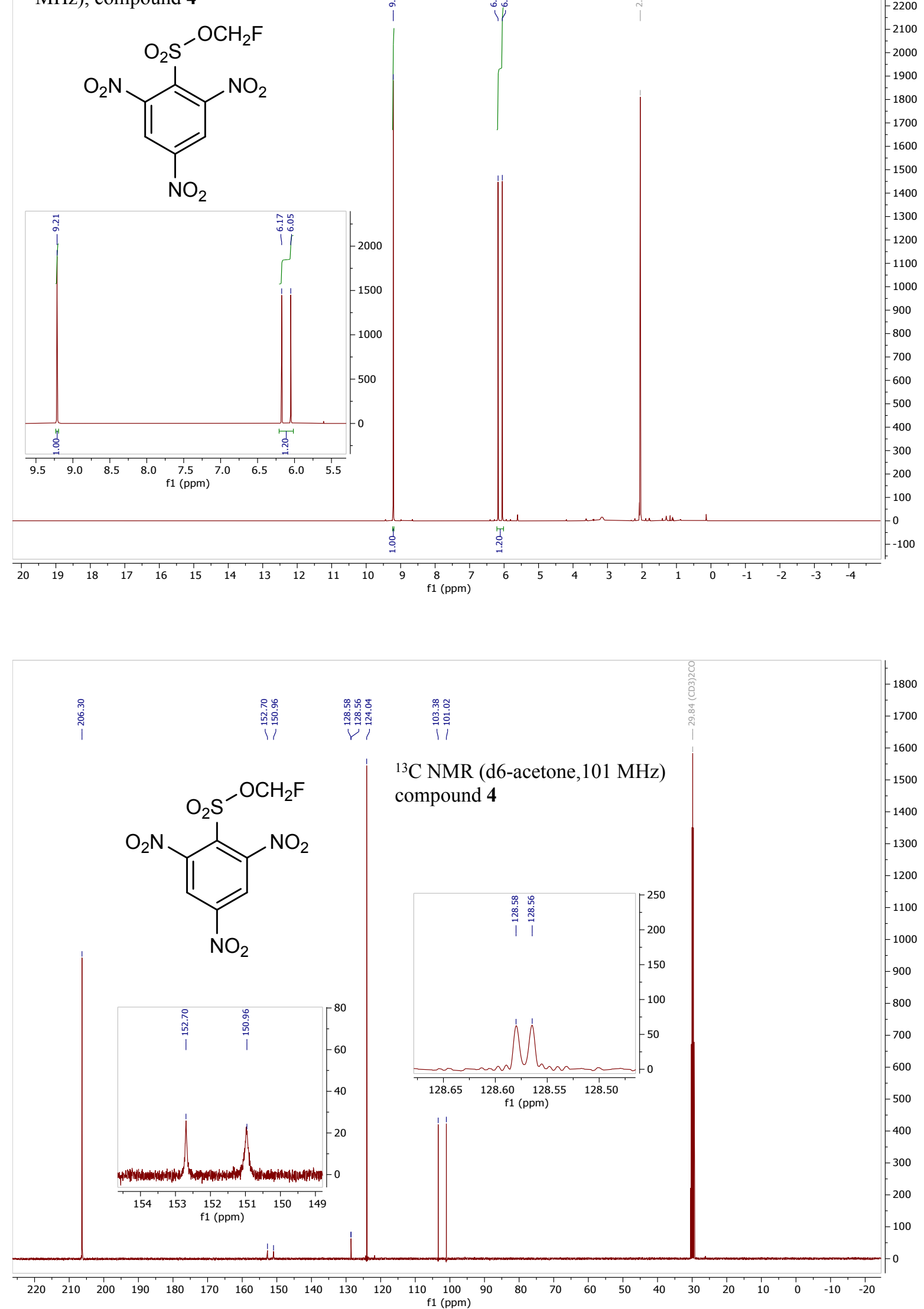

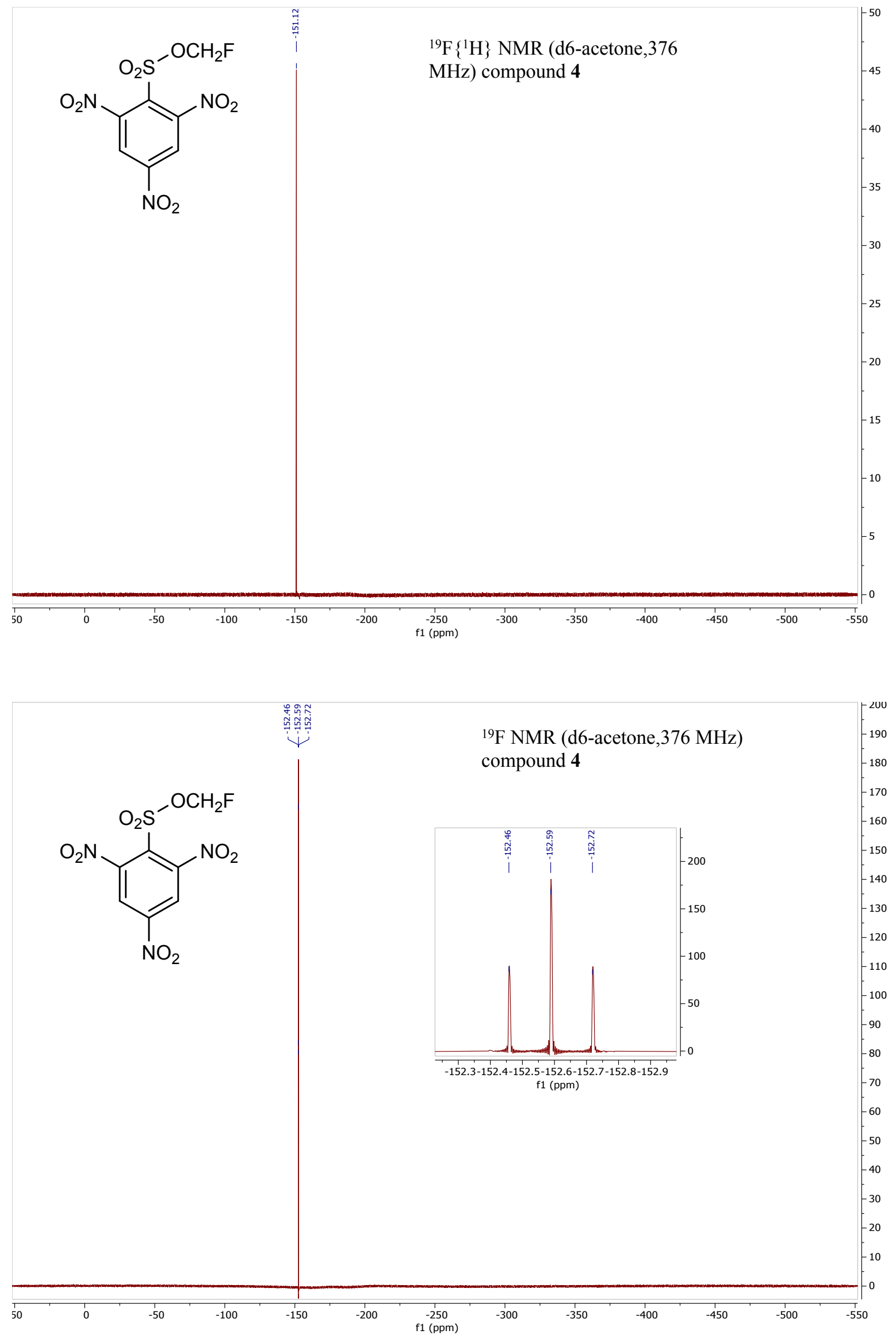


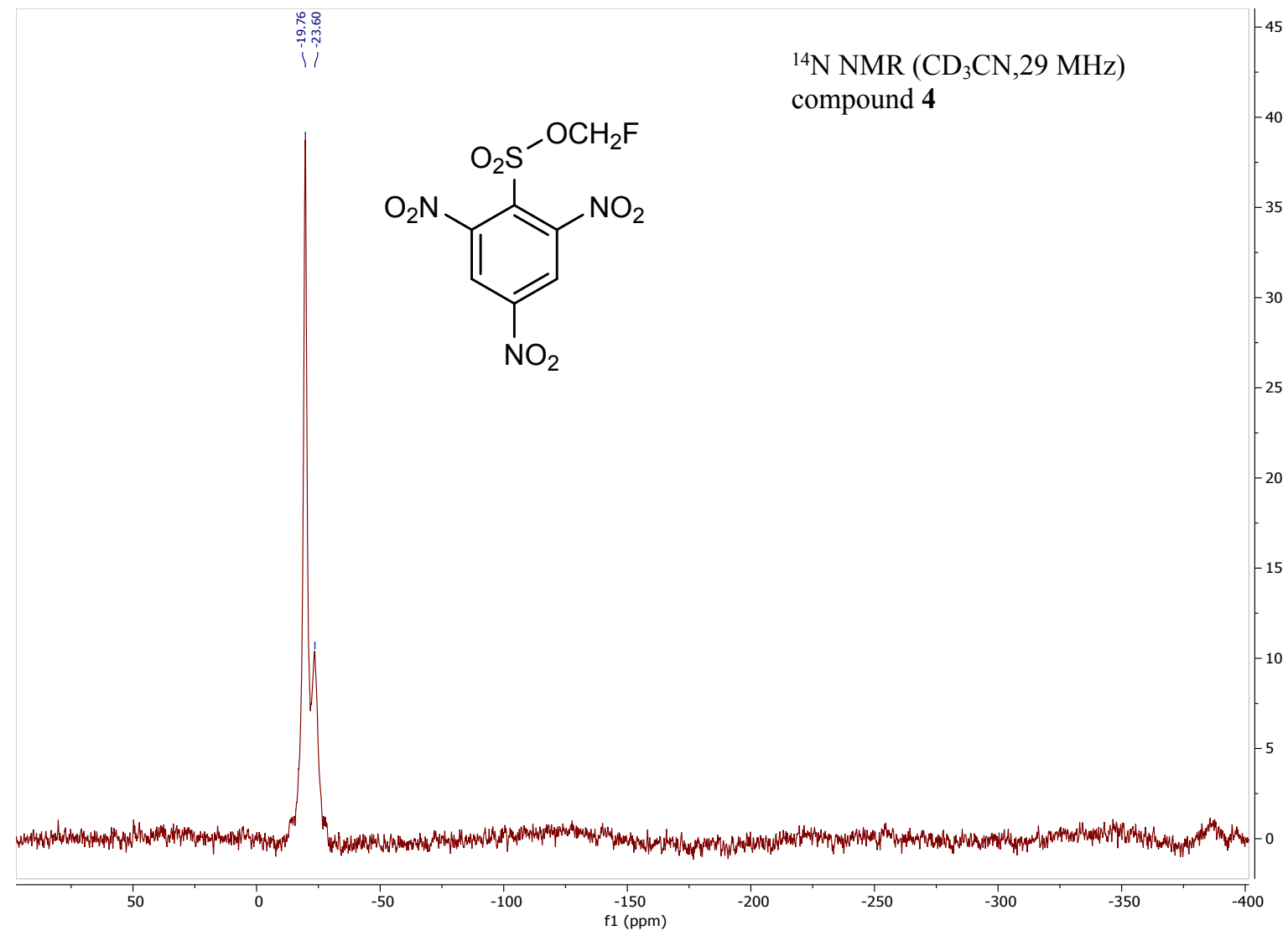

1.4 2-(Fluoromethyl)isouronium-2,4,6-trinitrobenzenesulfonate (5a)

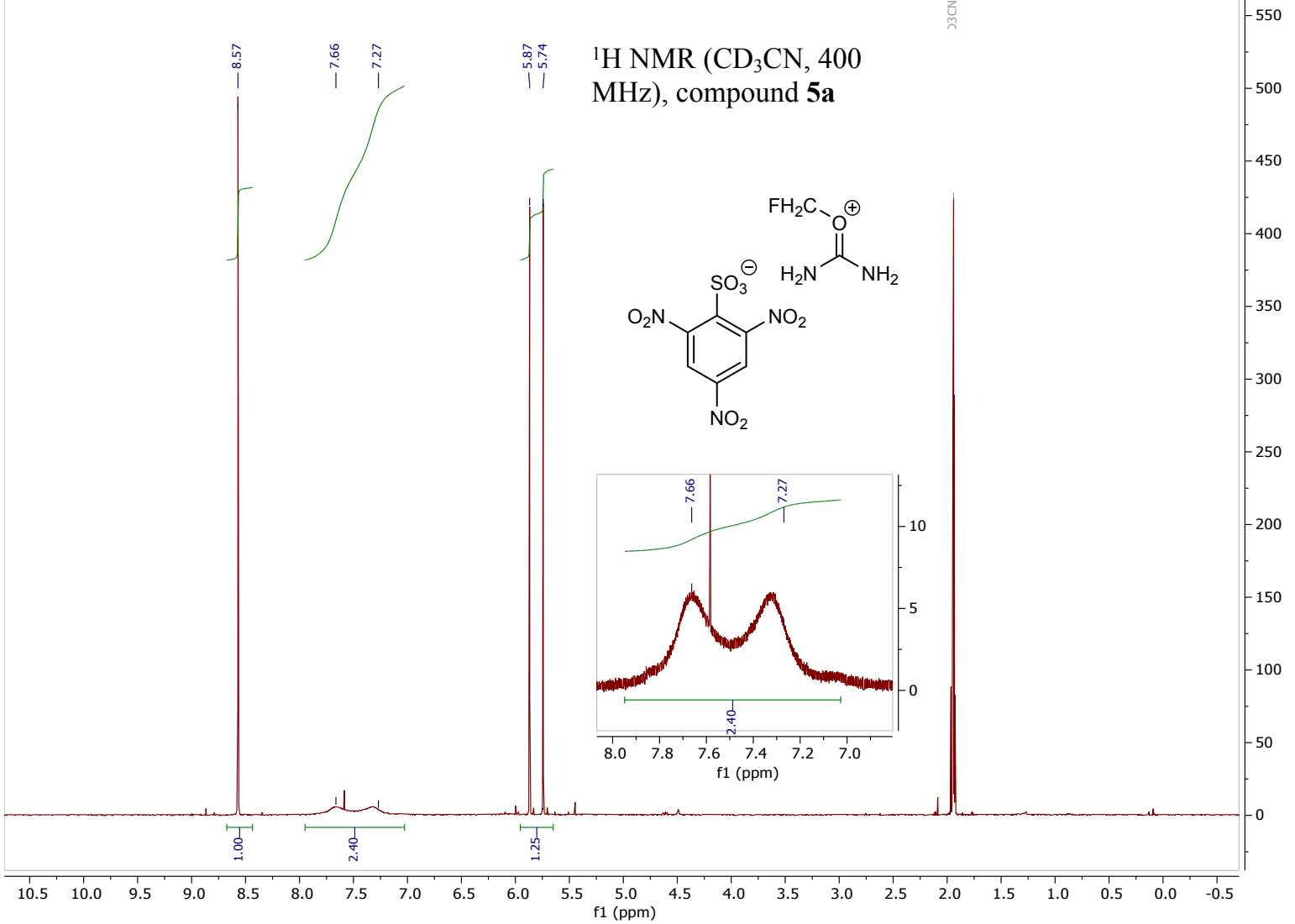



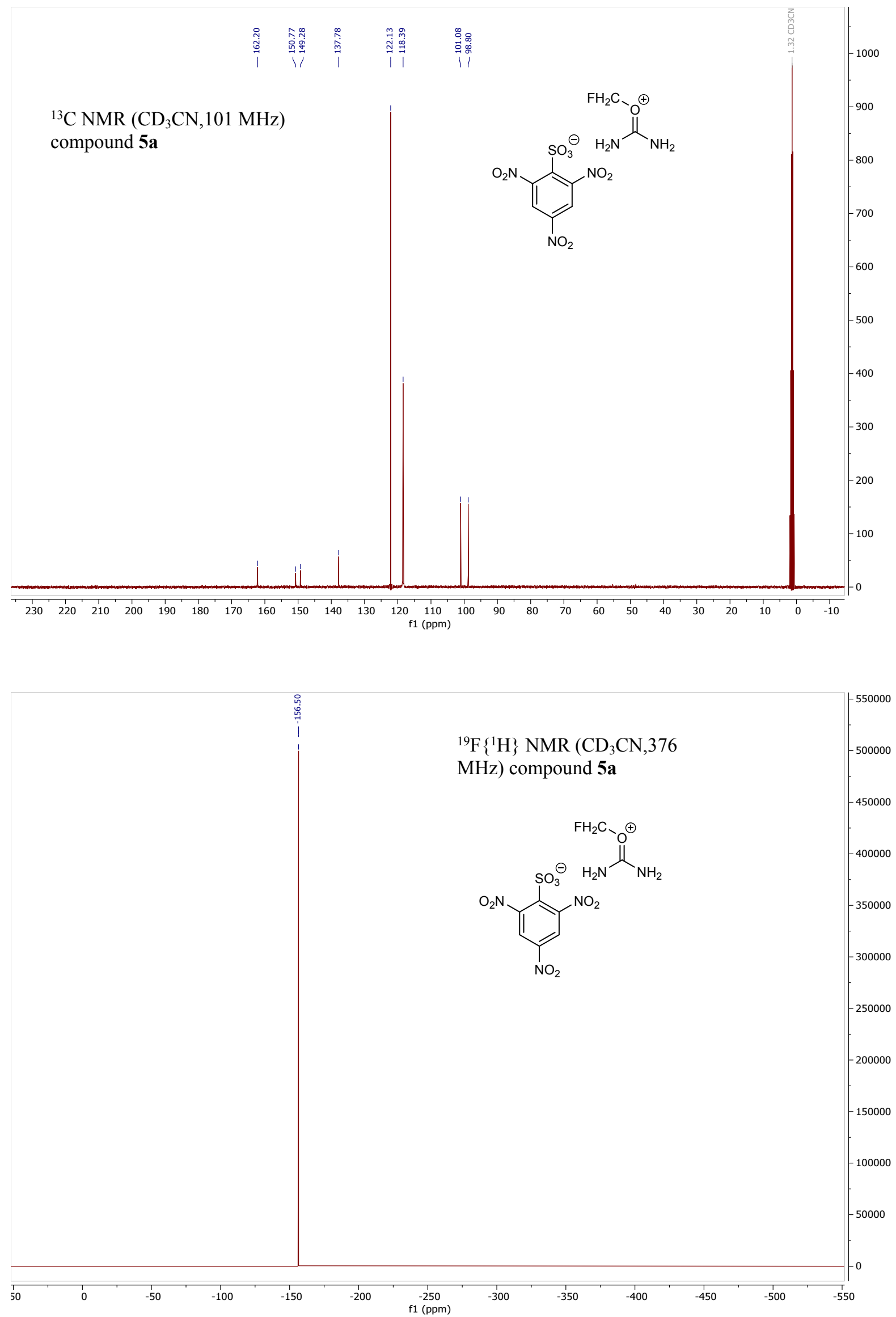

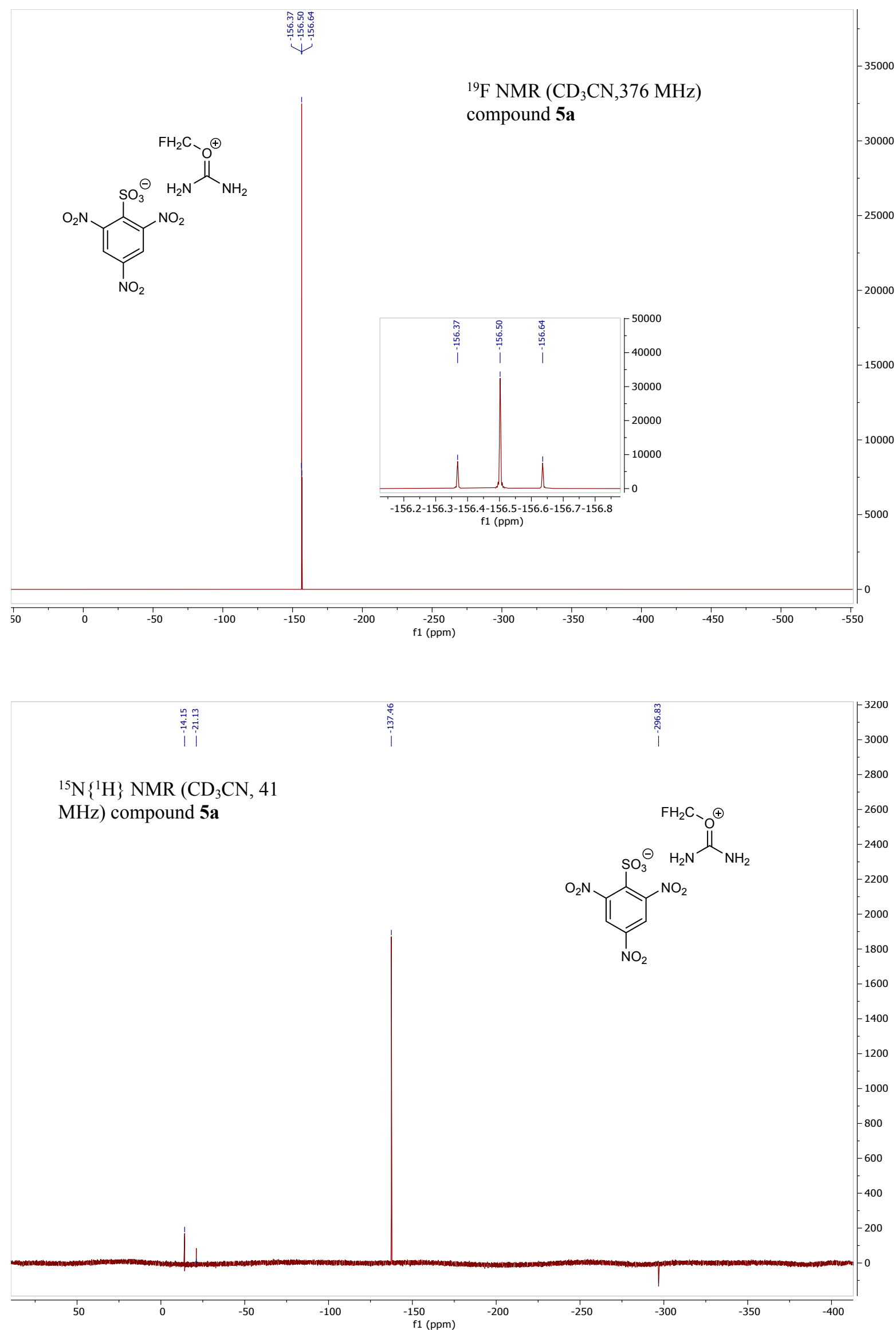
1.5 Ammonium-2,4,6-trinitrobenzenesulfonate (6a)
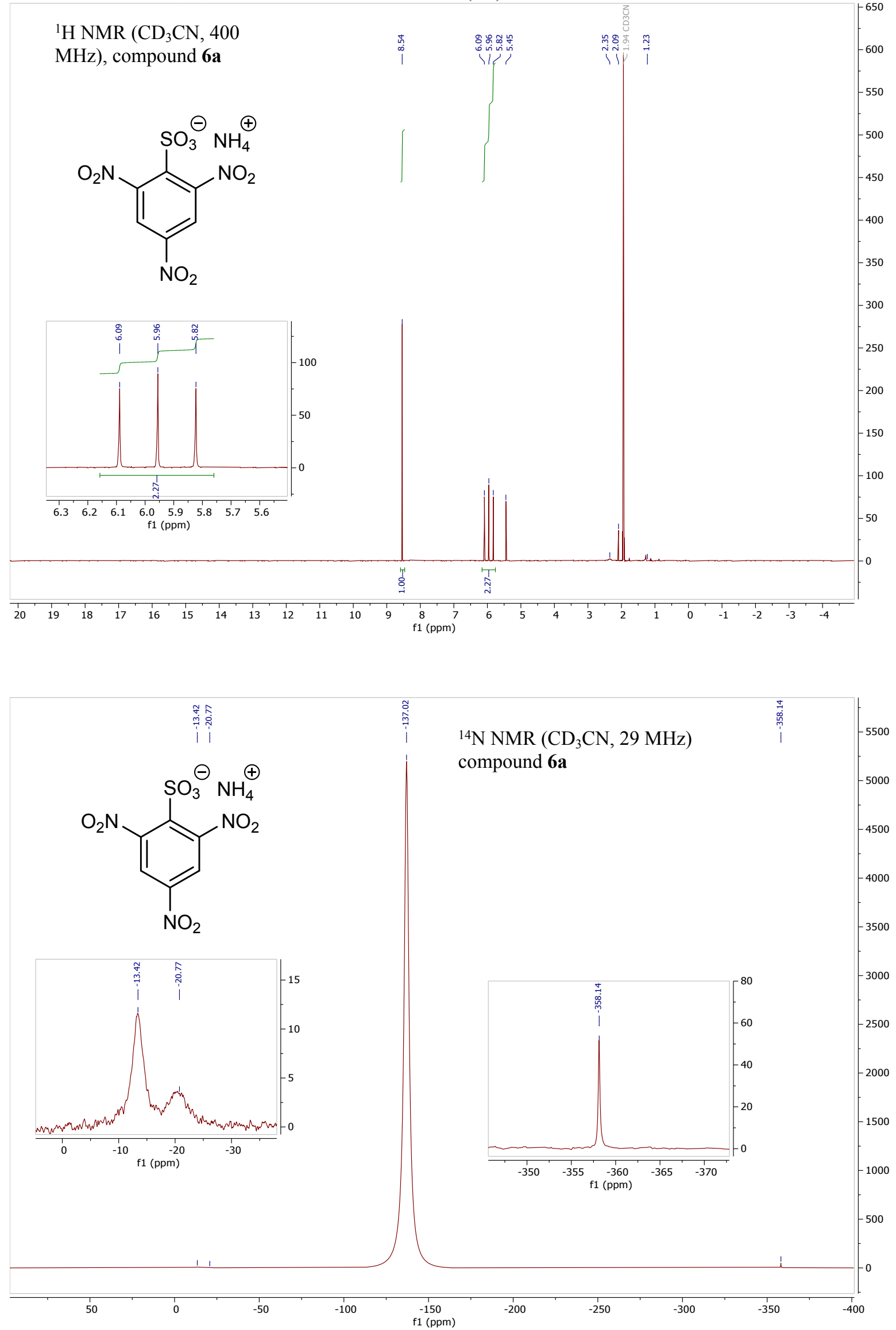
1.6 Dimethylacetamidiniumfluoromethyl-2,4,6-trinitrobenzenesulfonate (5b)
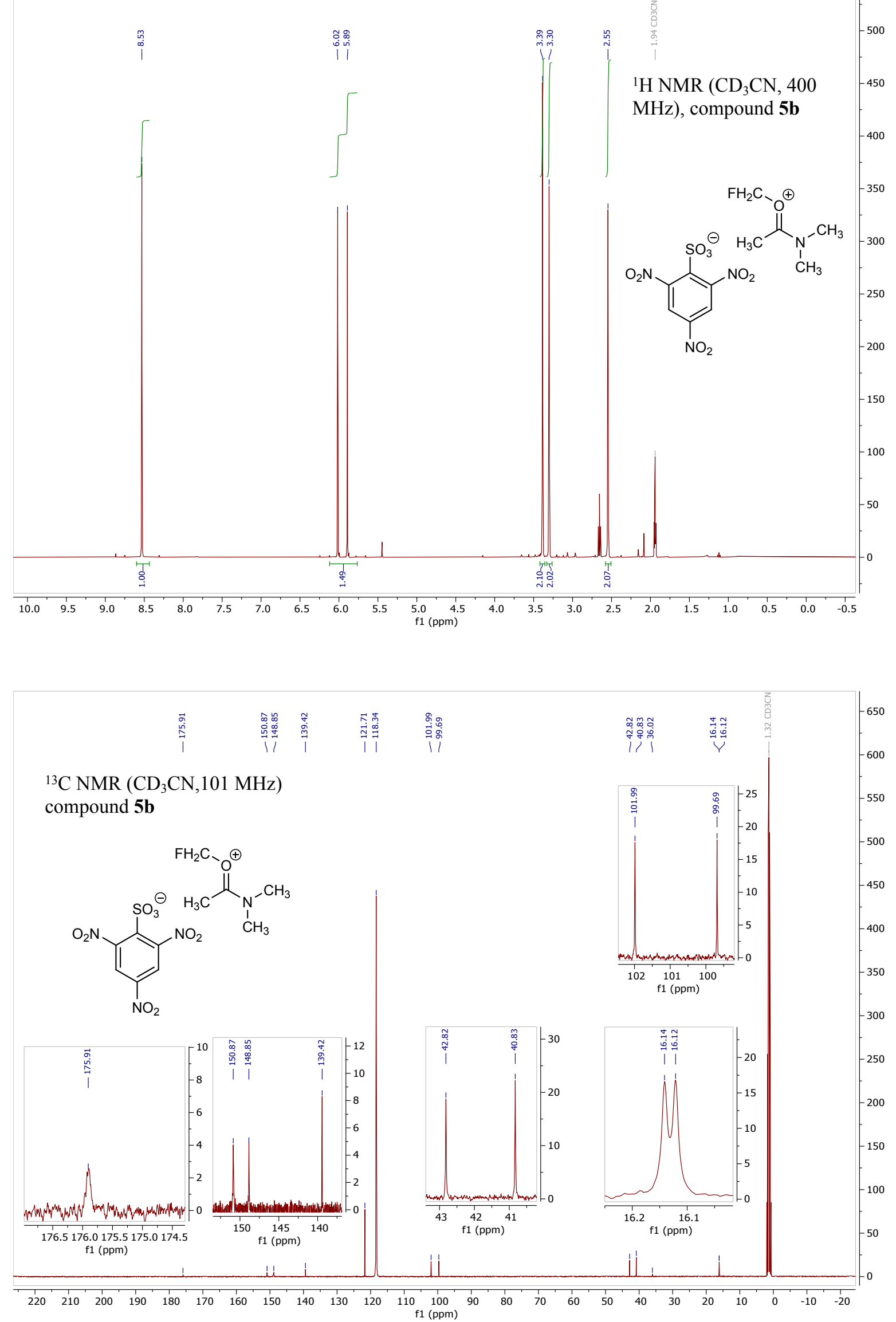

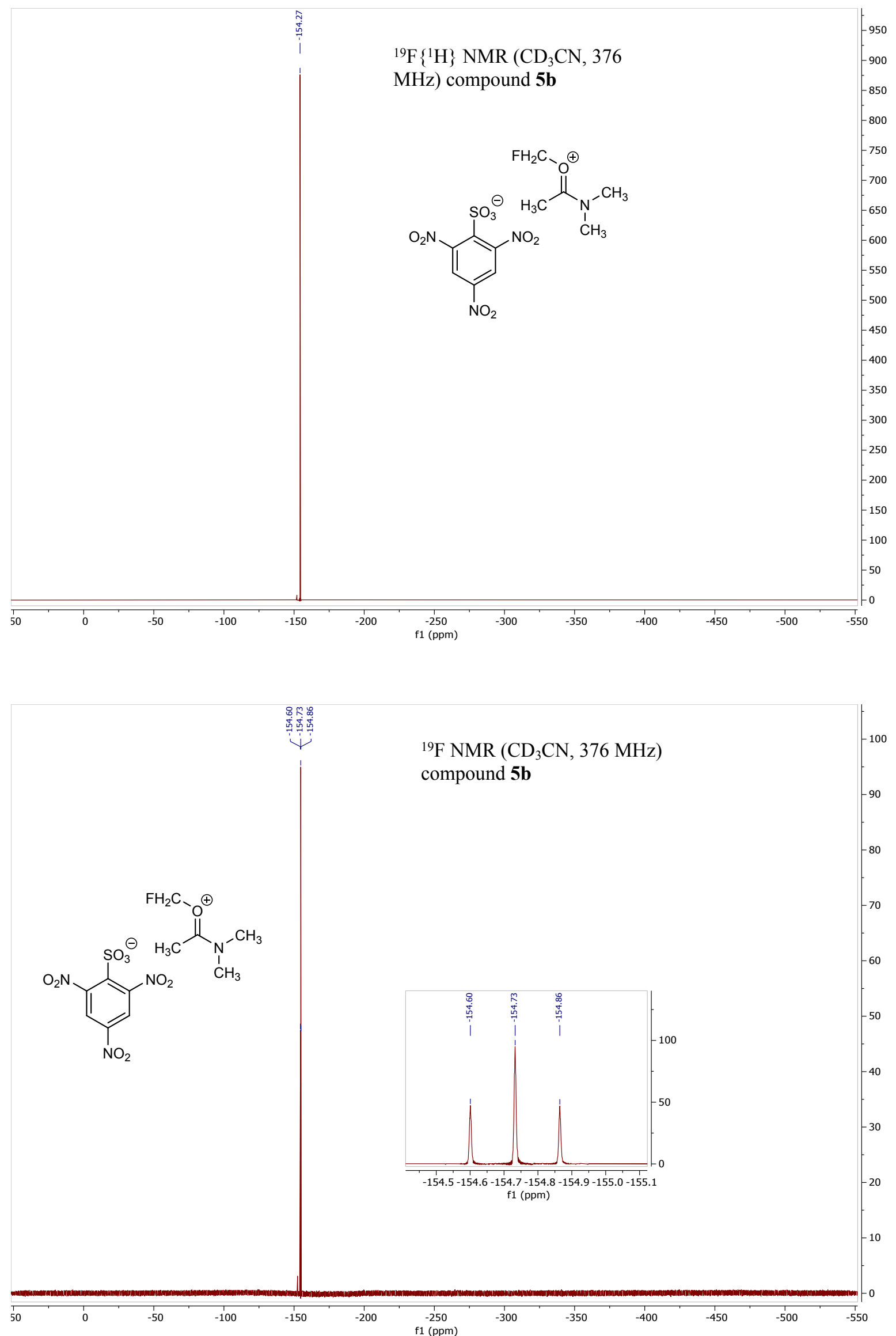


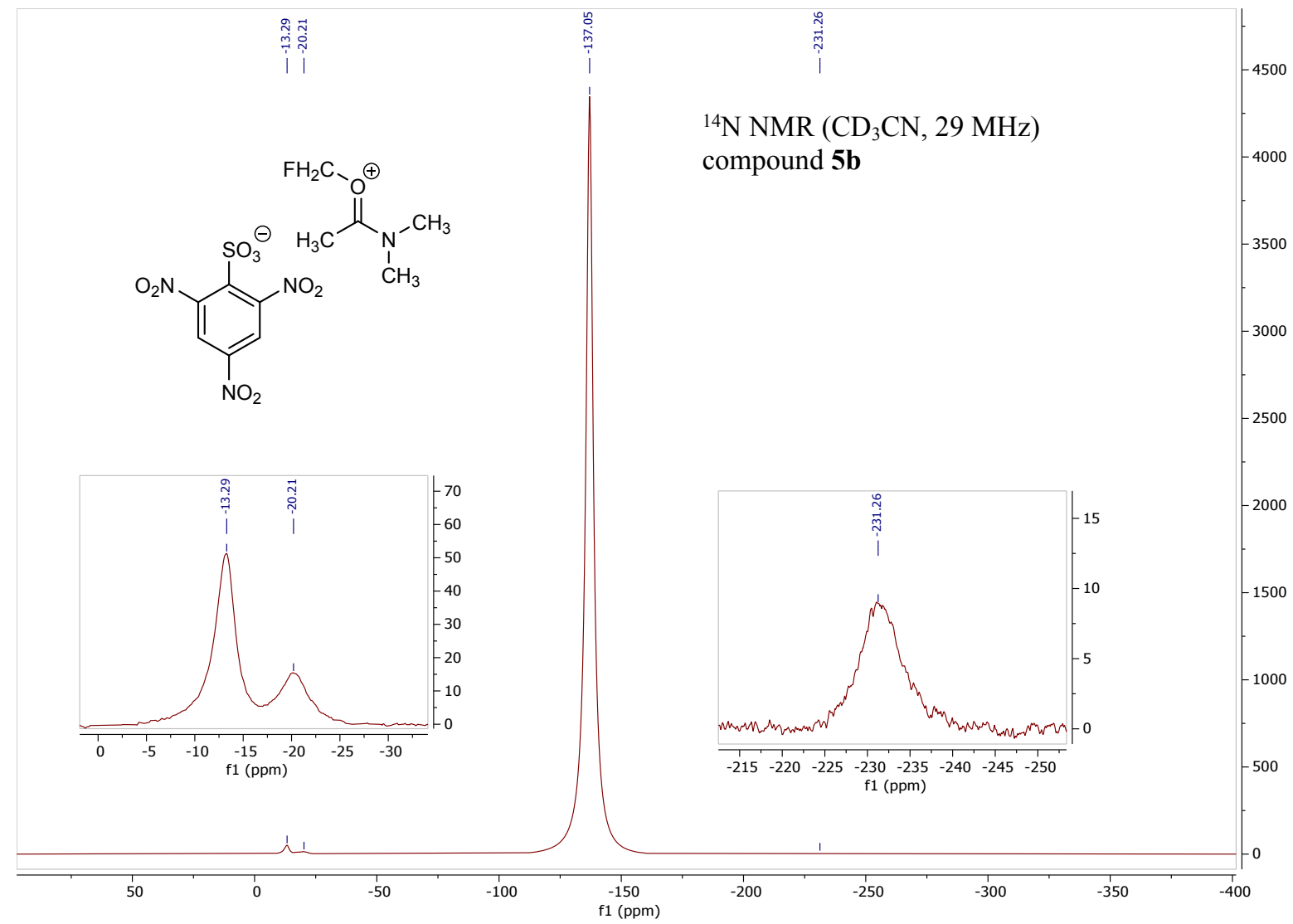

1.7 Dimethylformamidiniumfluoromethyl-2,4,6-trinitrobenzenesulfonate (5c)

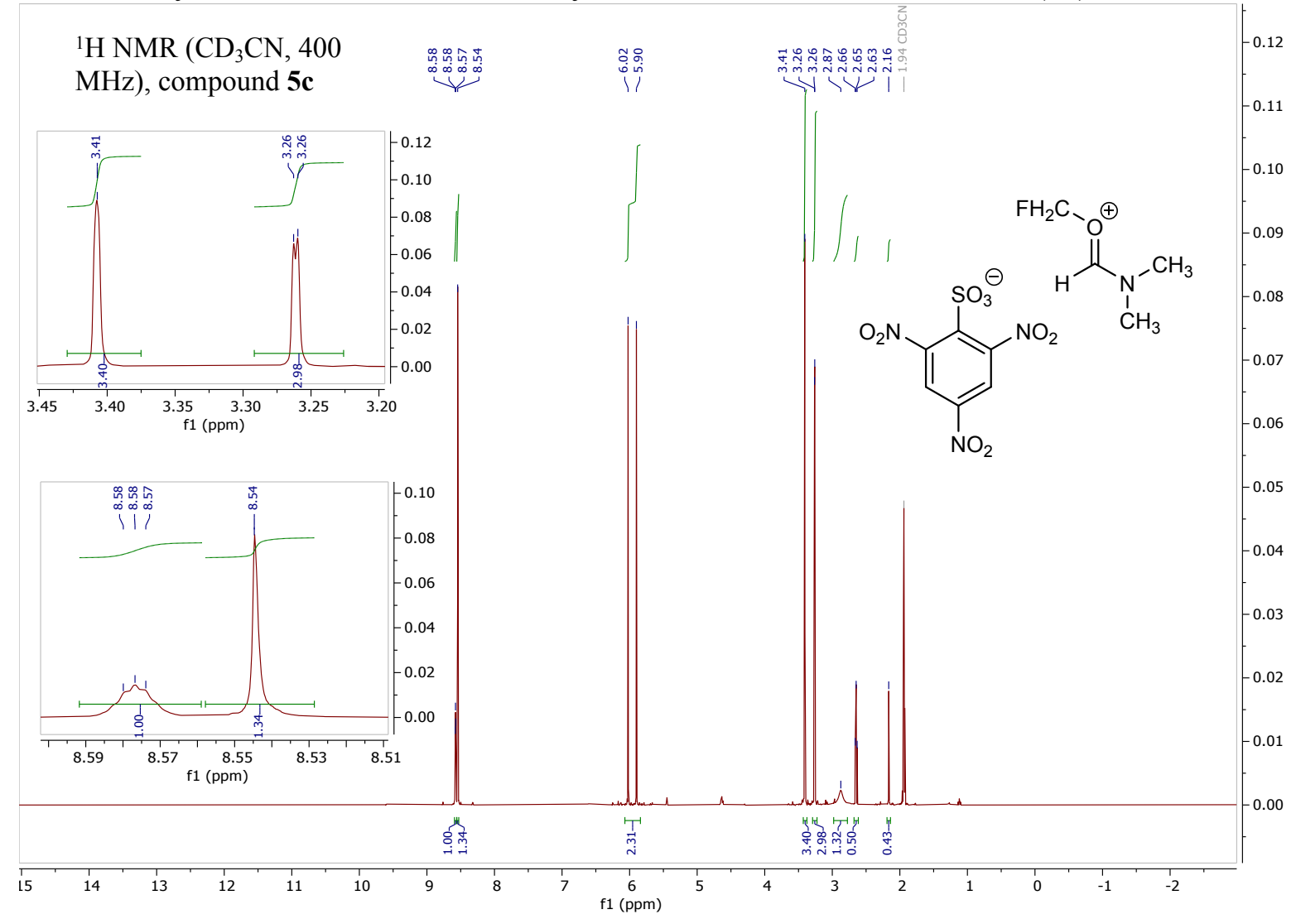




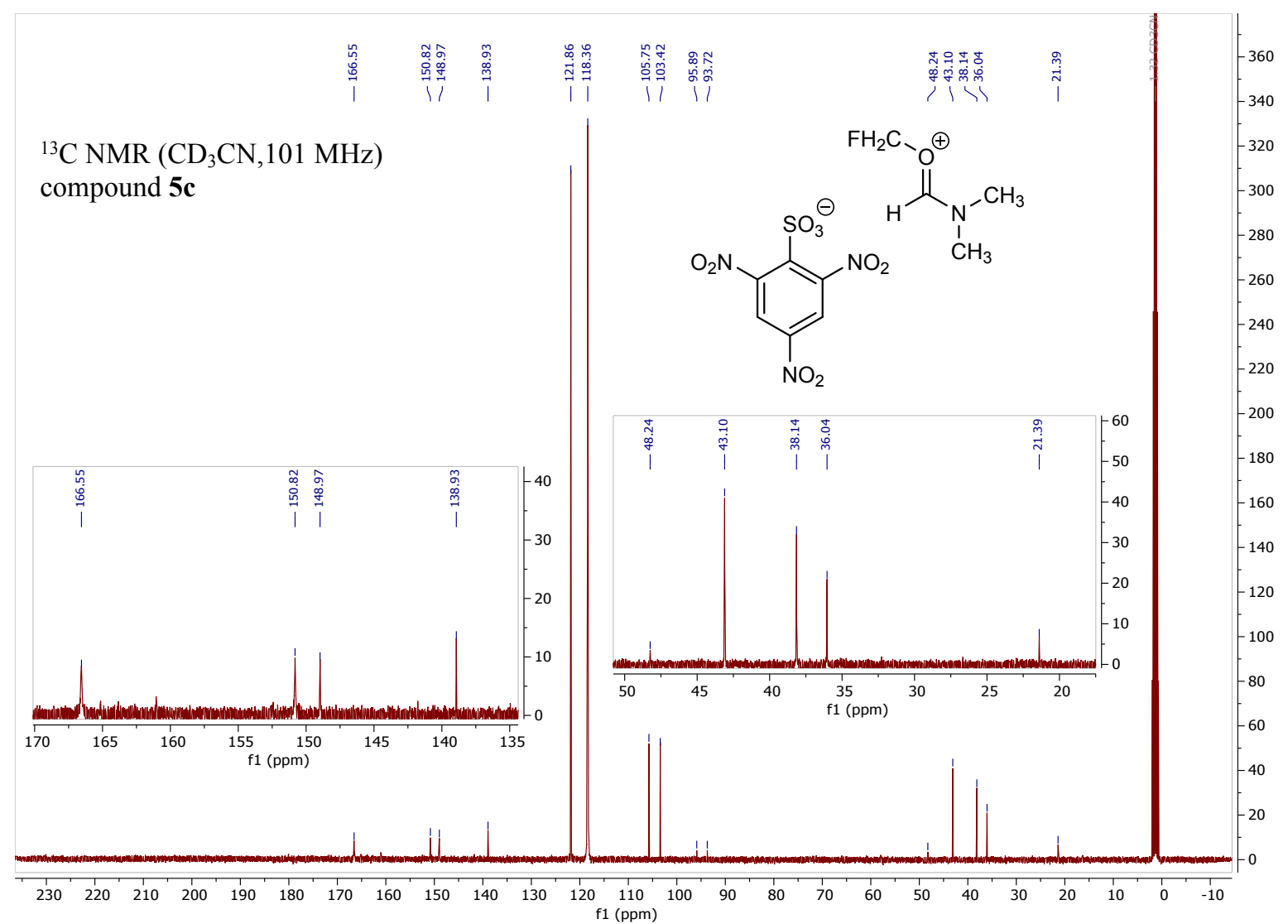

${ }^{19} \mathrm{~F}\left\{{ }^{1} \mathrm{H}\right\} \mathrm{NMR}\left(\mathrm{CD}_{3} \mathrm{CN}, 376\right.$

$\mathrm{MHz}$ ) compound $\mathbf{5 c}$

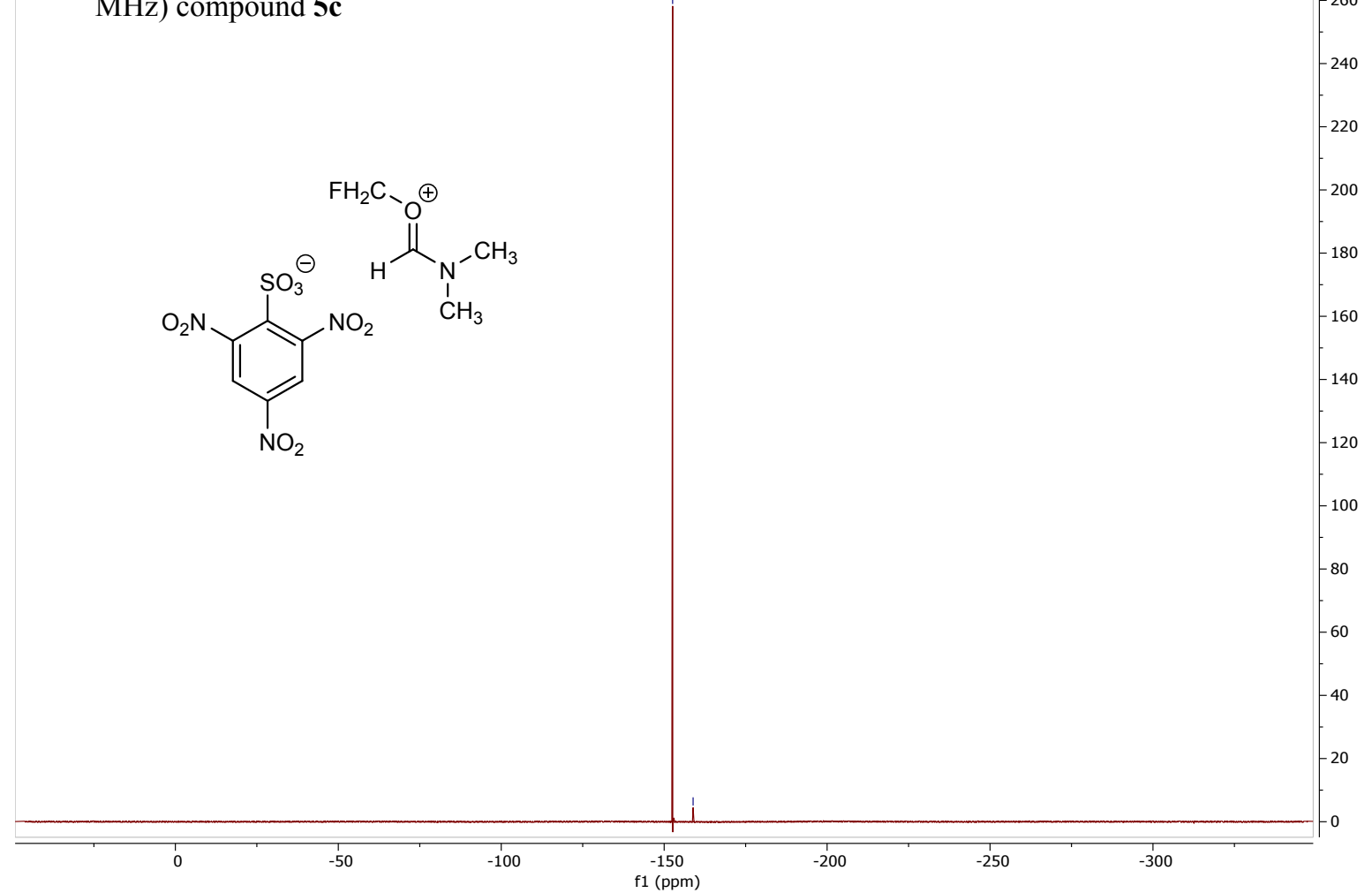




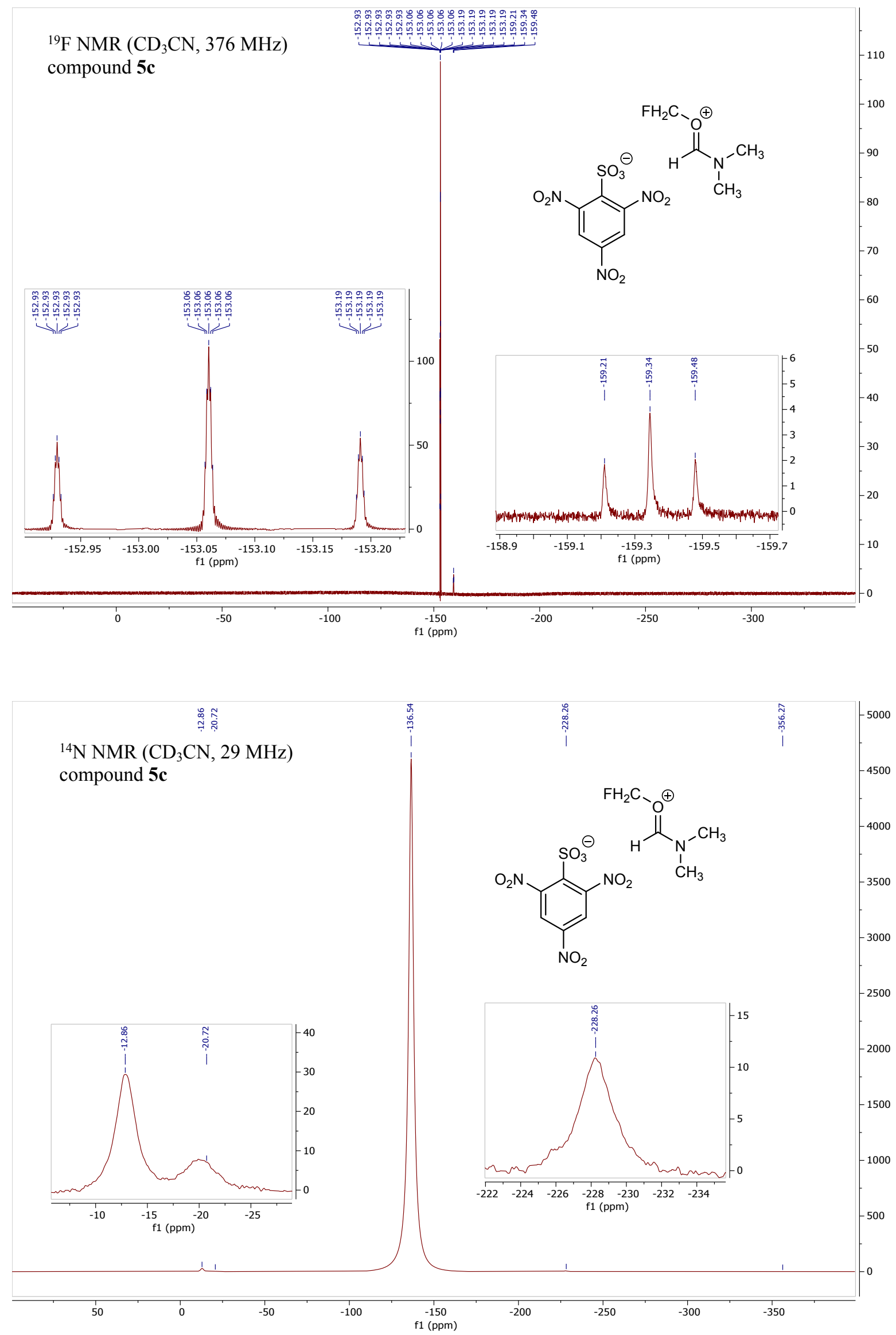


1.8 Dimethylammonium-2,4,6-trinitrobenzenesulfonate $(\mathbf{6 b})$
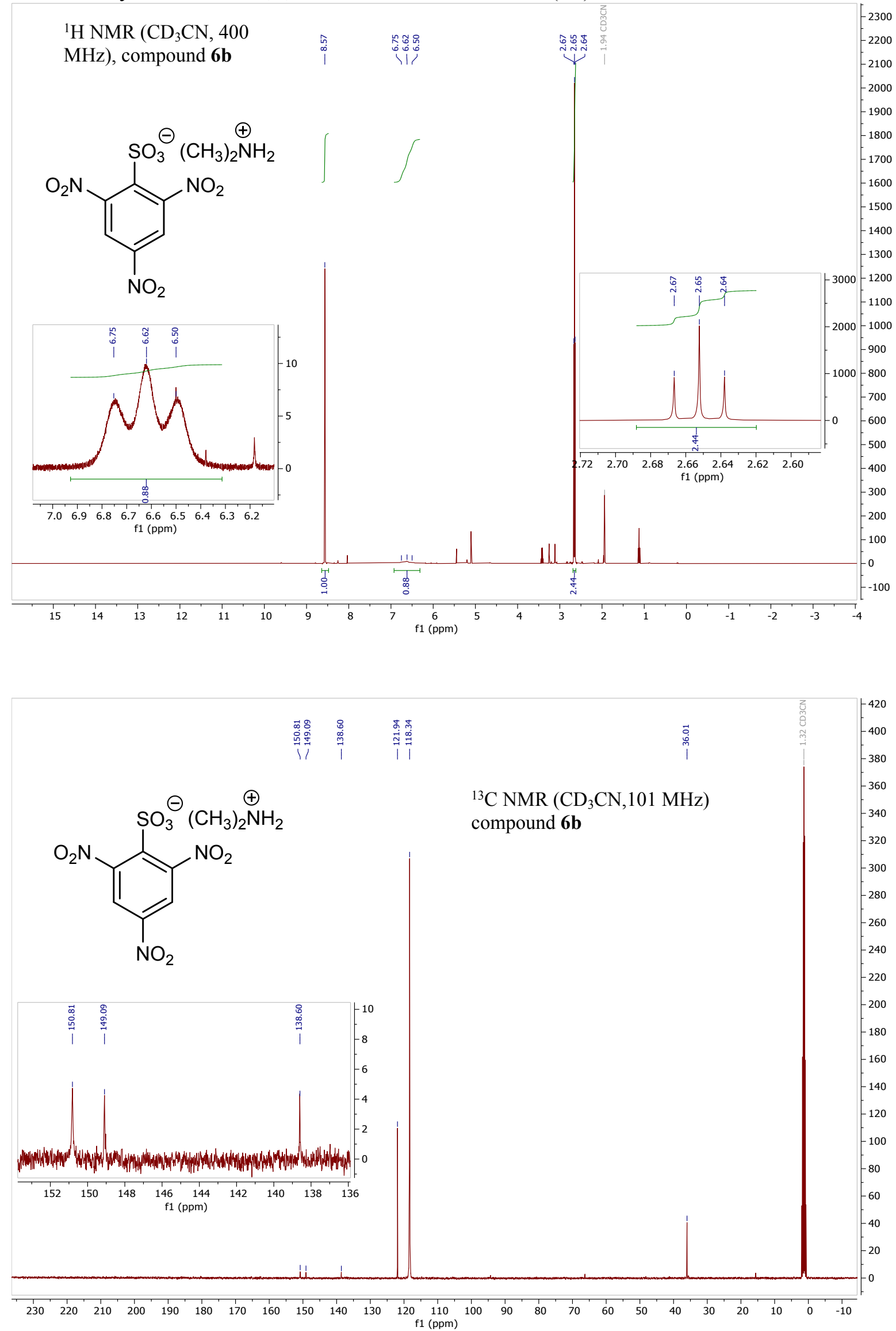


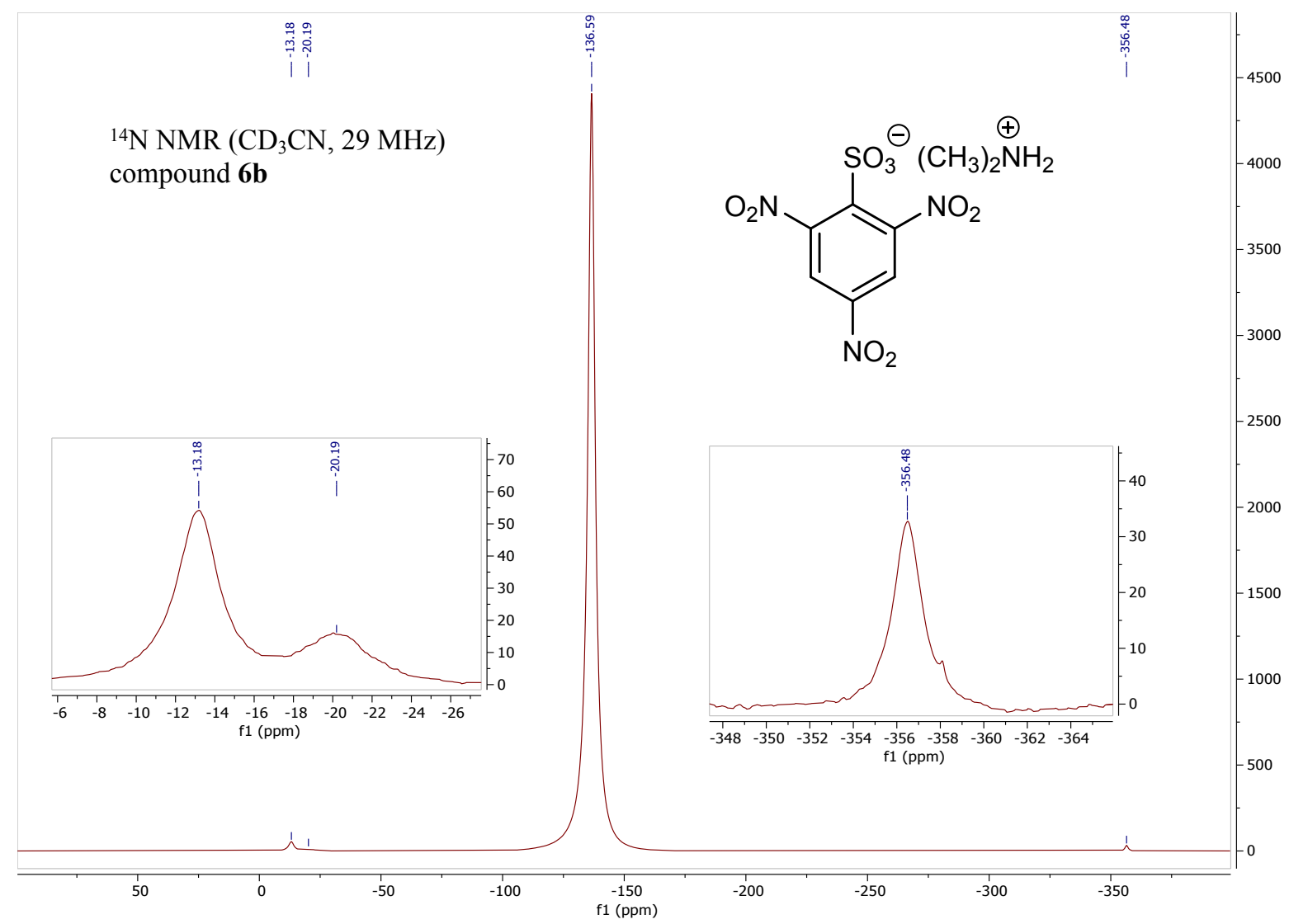

1.9 Dimethylammonium- $\mathrm{d}_{2}-2,4,6$-trinitrobenzenesulfonate $(\mathbf{6 b})$

${ }^{1} \mathrm{H}$ NMR $\left(\mathrm{D}_{2} \mathrm{O}, 400\right.$

$\mathrm{MHz}$ ), compound $\mathbf{6 b}$ with $\mathrm{D}_{2} \mathrm{O}$<smiles>CS(=O)(=O)c1c([N+](=O)[O-])cc([N+](=O)[O-])cc1[N+](=O)[O-]</smiles>
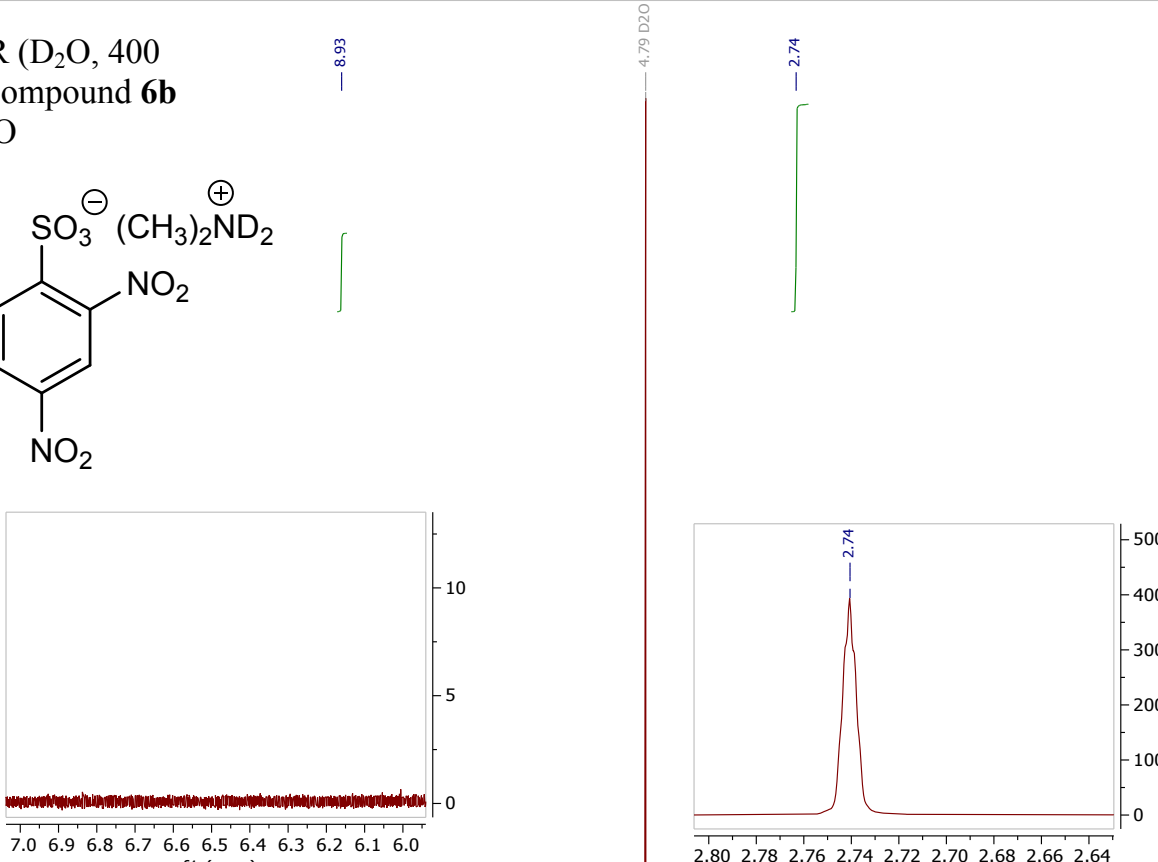

3000

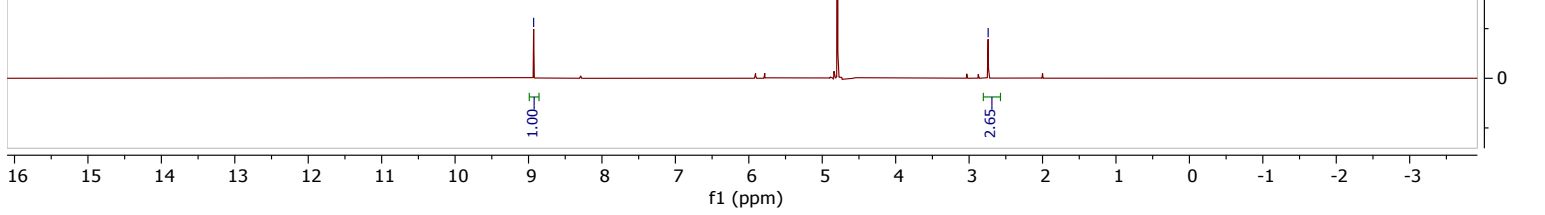


1.10 Dimethyl-2,4,6-trinitroaniline
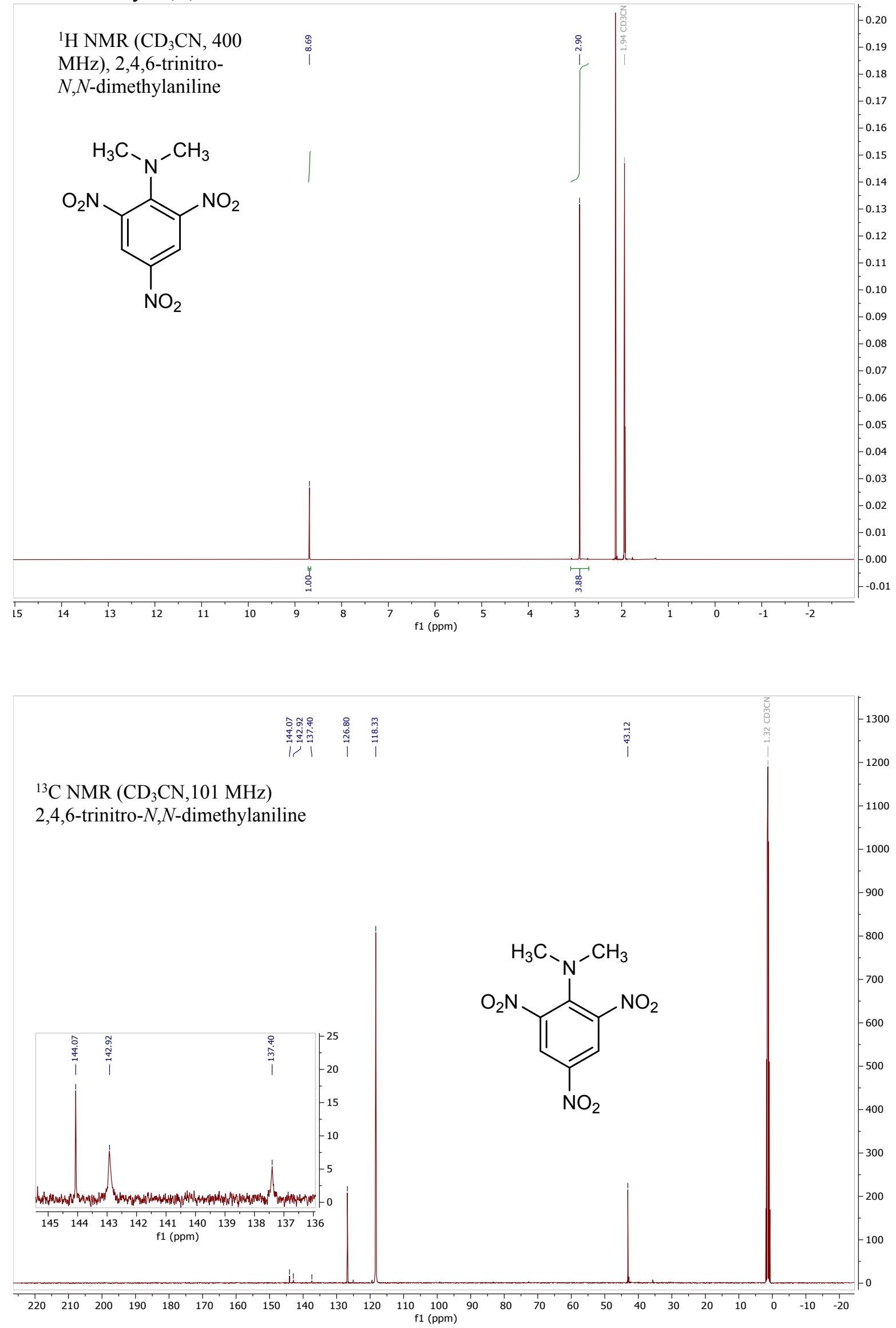
1.7 Fluoromethylthiotriphenylphosphonium-2,4,6-trinitrobenzenesulfonate (7b)
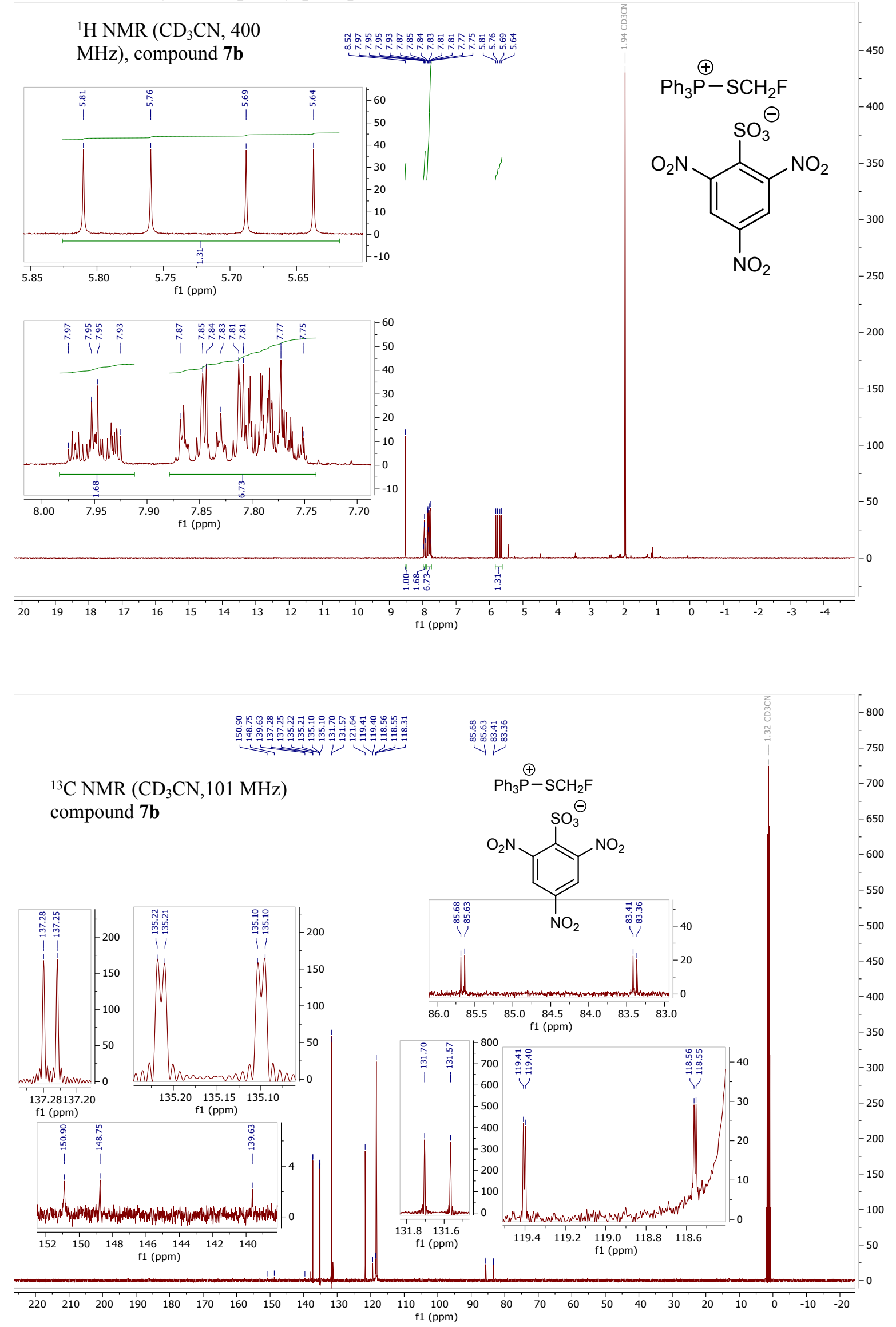
${ }^{19} \mathrm{~F}\left\{{ }^{1} \mathrm{H}\right\}$ NMR $\left(\mathrm{CD}_{3} \mathrm{CN}, 376\right.$

$\mathrm{MHz}$ ) compound $\mathbf{7 b}$

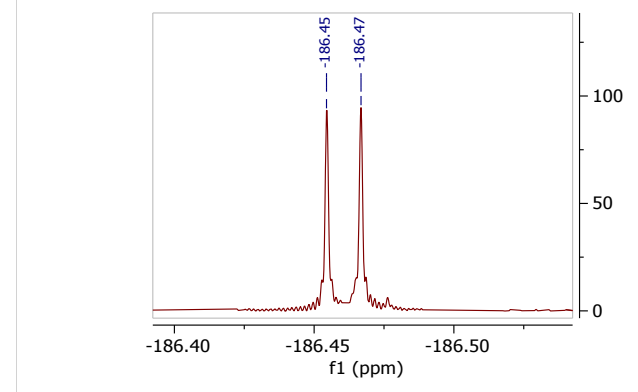

$\oplus$

$\mathrm{Ph}_{3} \mathrm{P}-\mathrm{SCH}_{2} \mathrm{~F}$

(NO2)

$1+50$

$-100$

${ }^{19} \mathrm{~F} \mathrm{NMR}\left(\mathrm{CD}_{3} \mathrm{CN}, 376 \mathrm{MHz}\right)$

compound $\mathbf{7 b}$

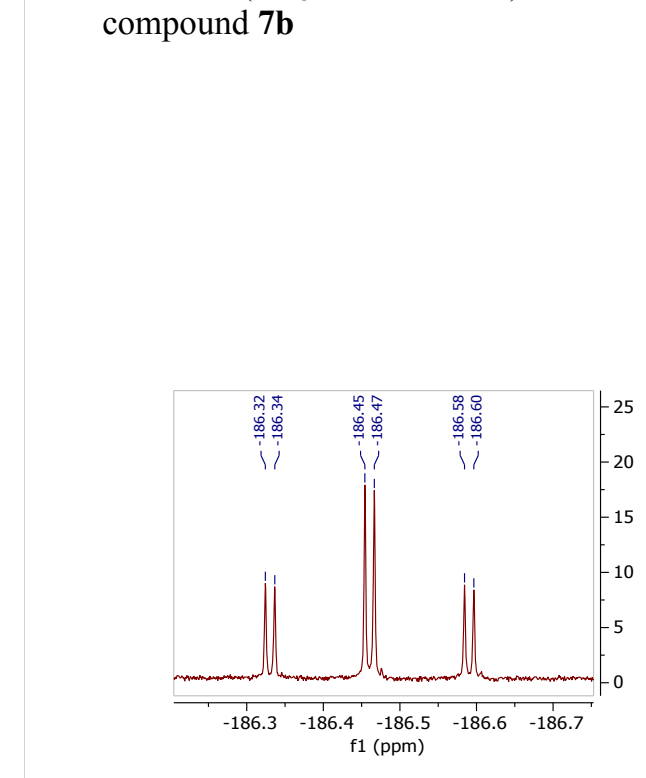

$-200$

$-250$

$-300$ 


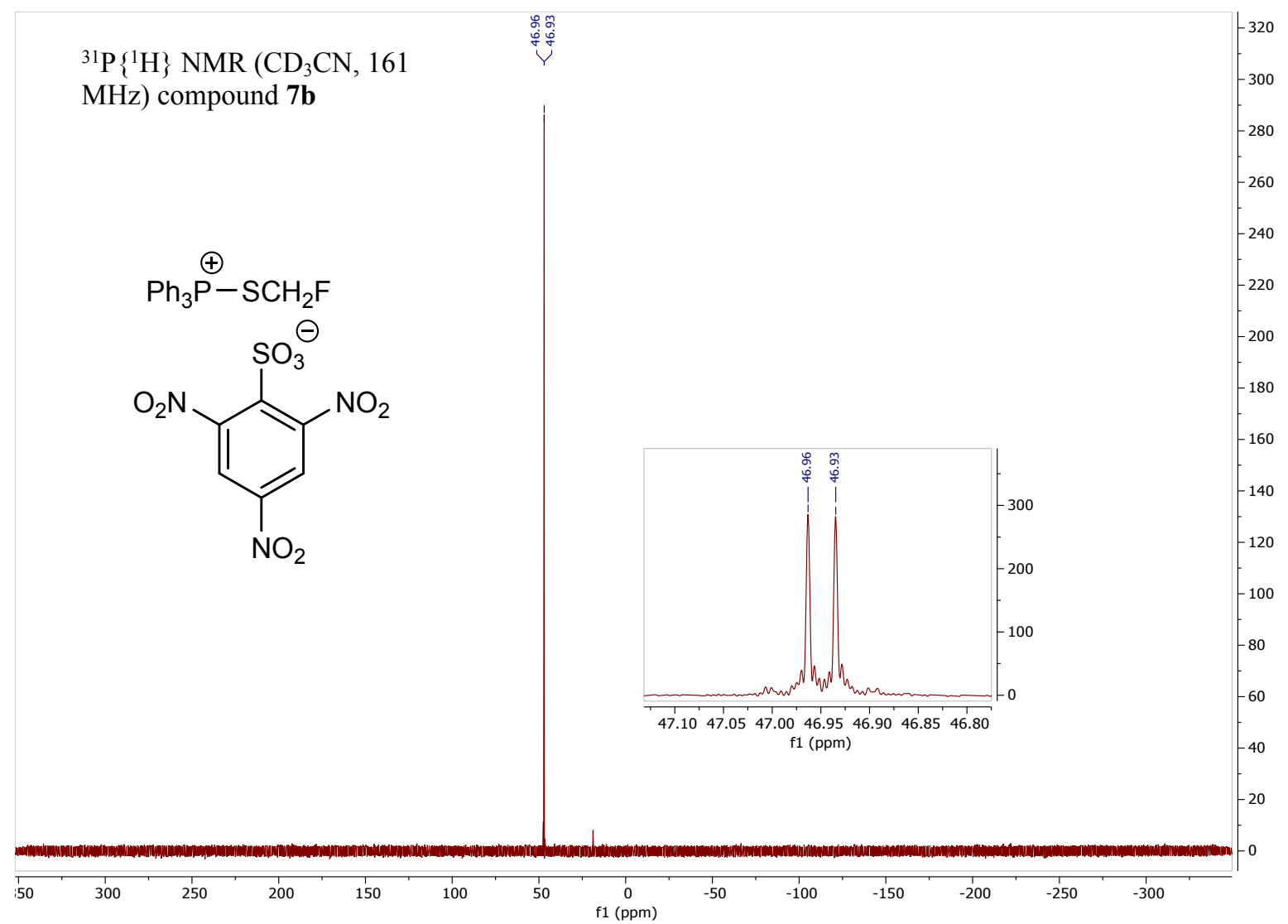

${ }^{31} \mathrm{P}$ NMR $\left(\mathrm{CD}_{3} \mathrm{CN}, 161 \mathrm{MHz}\right)$ compound $\mathbf{7 b}$
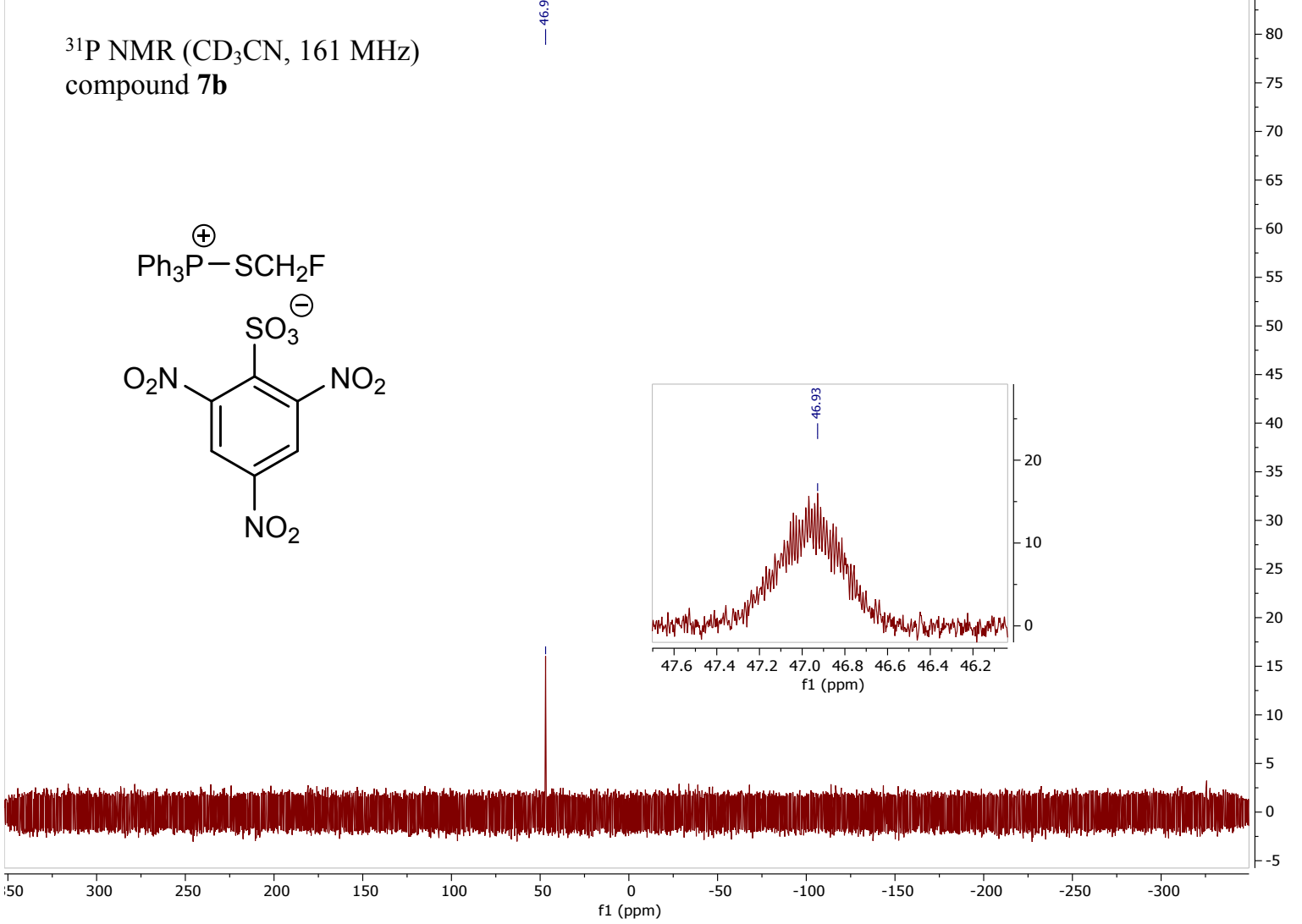
1.12 Fluoromethylselenotriphenylphosphonium-2,4,6-trinitrobenzenesulfonate (7c)

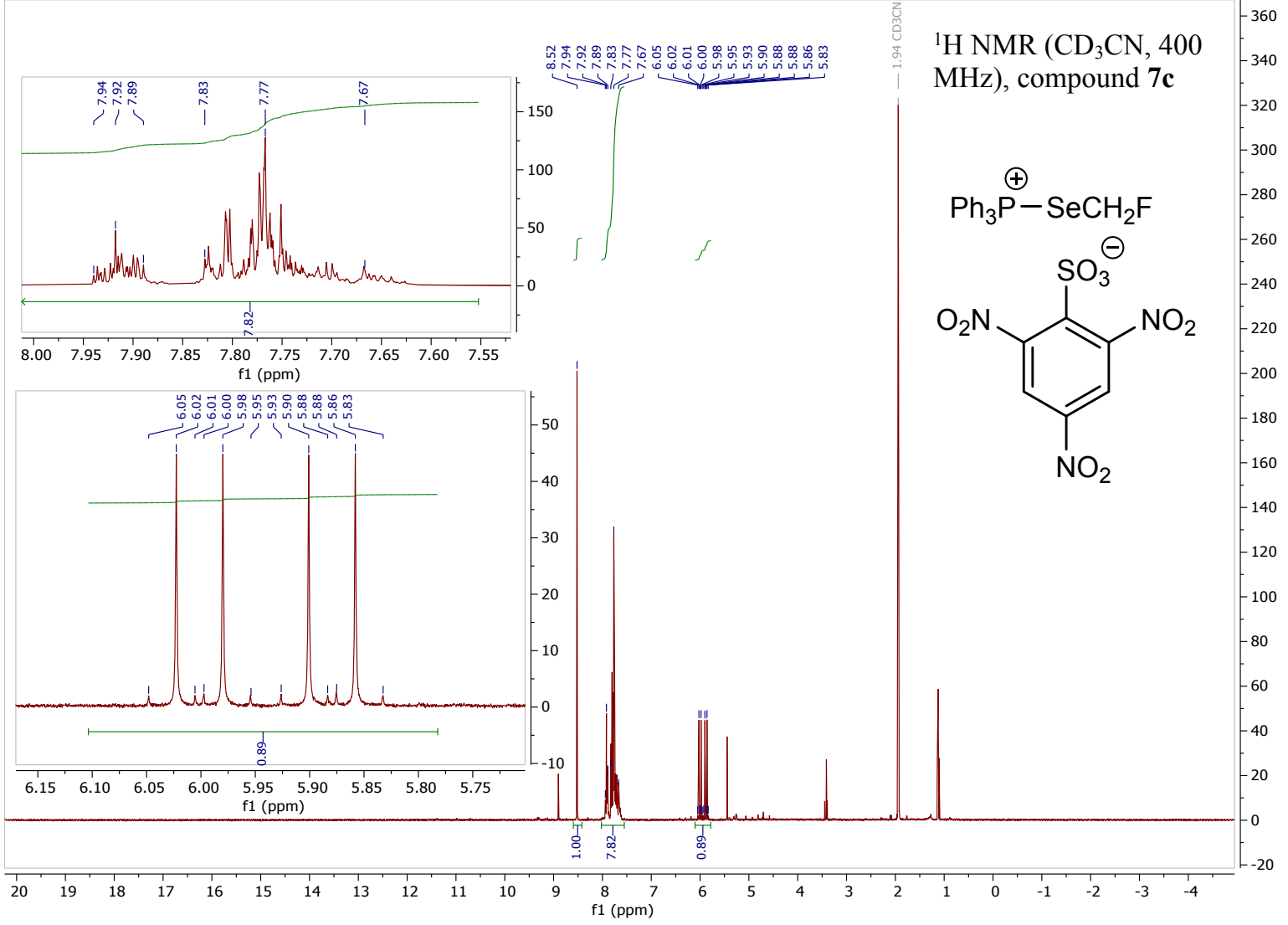

の

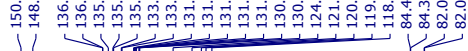

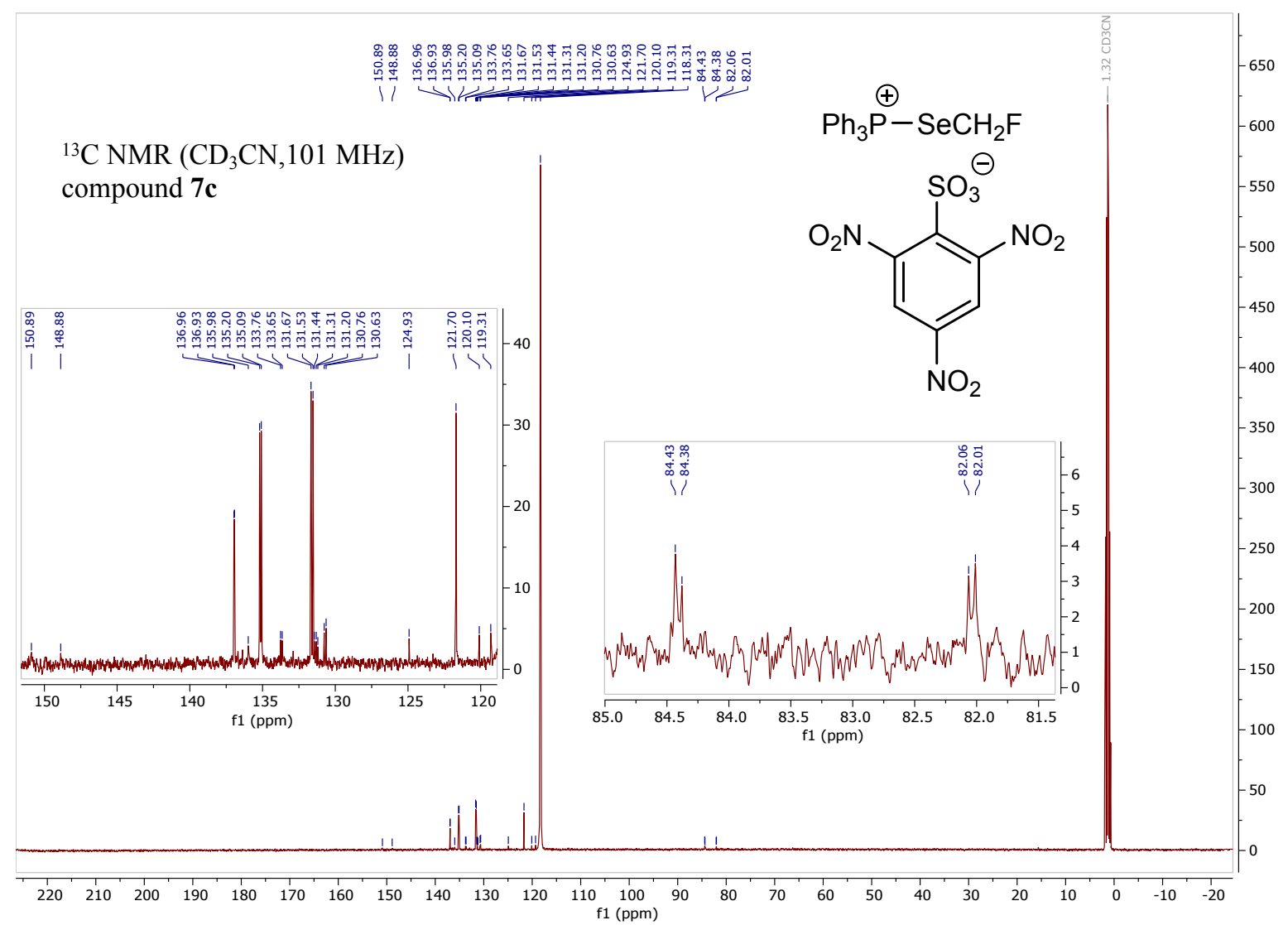




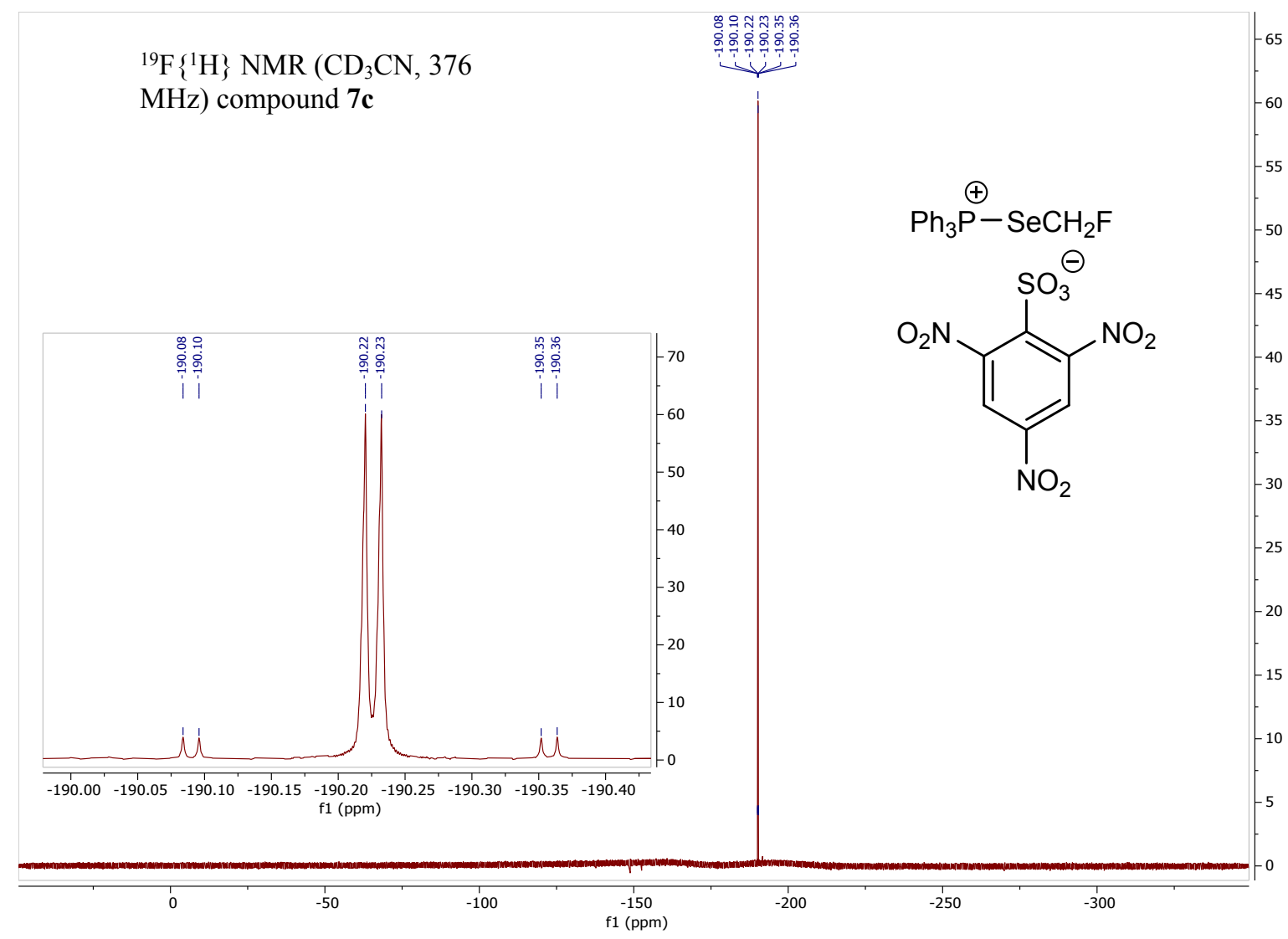

${ }^{19} \mathrm{~F}$ NMR $\left(\mathrm{CD}_{3} \mathrm{CN}, 376 \mathrm{MHz}\right)$

compound $7 \mathrm{c}$
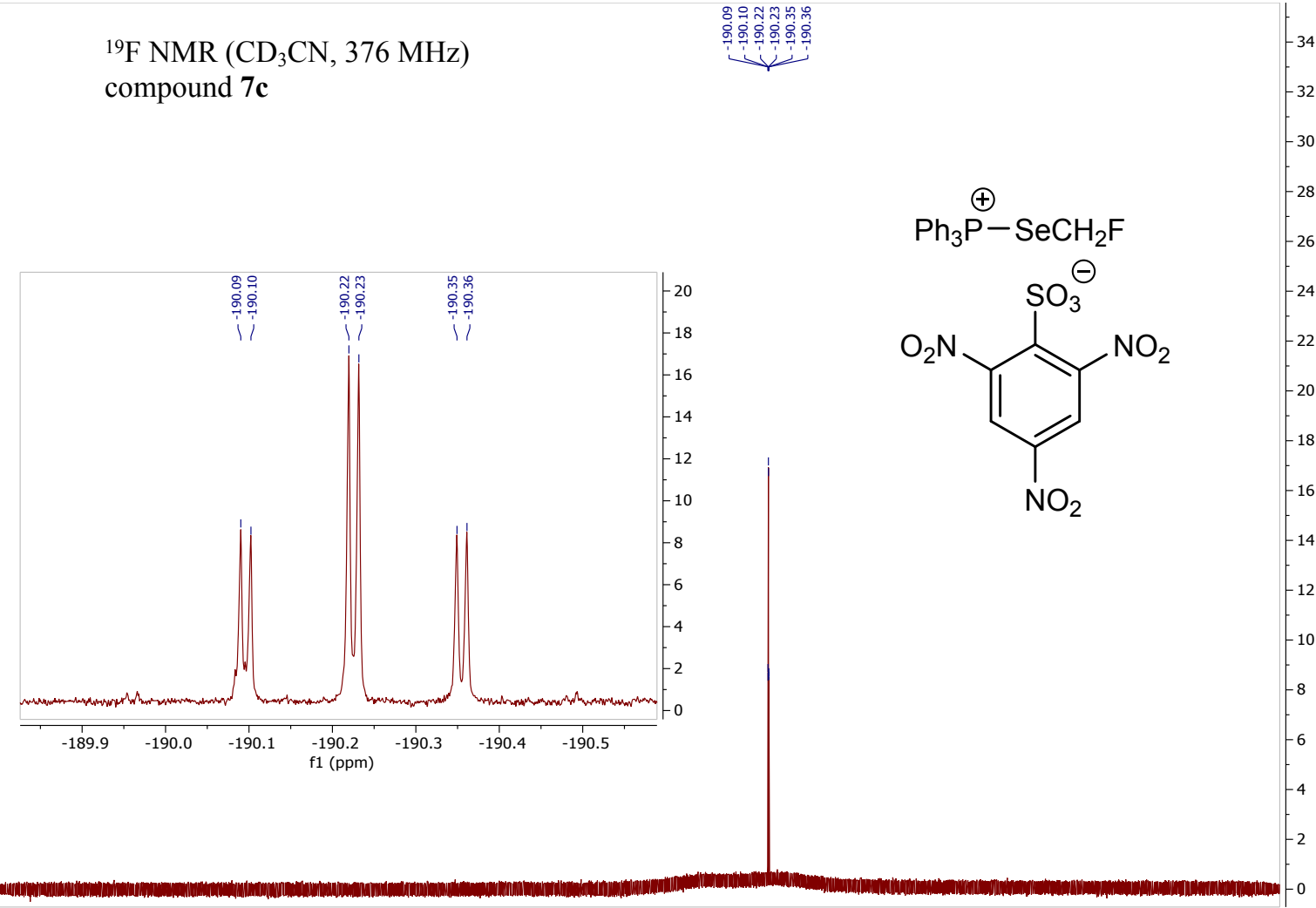

$-250$ 


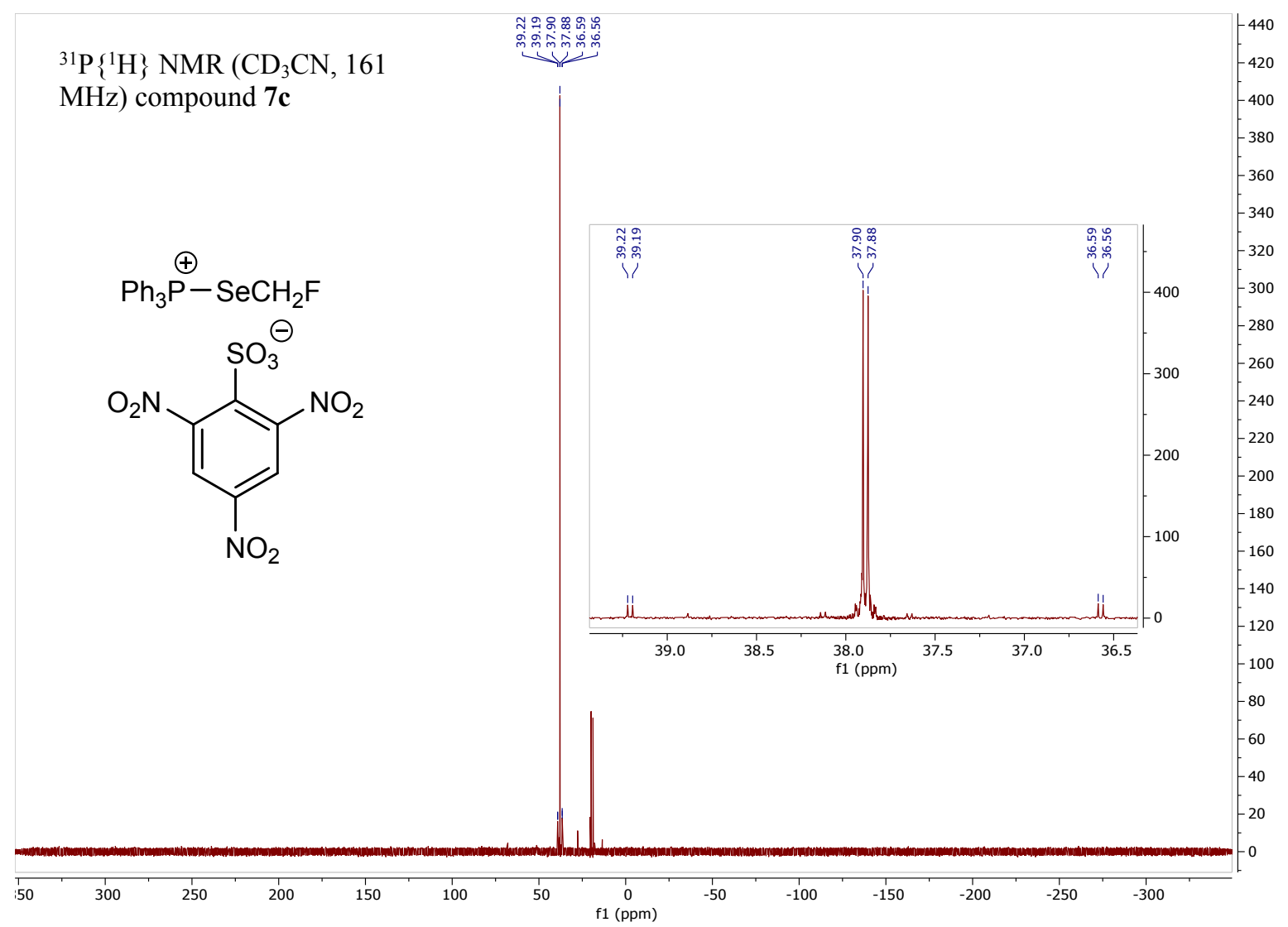

${ }^{77} \mathrm{Se}\left\{{ }^{1} \mathrm{H}\right\} \mathrm{NMR}\left(\mathrm{CD}_{3} \mathrm{CN}, 76\right.$

$\mathrm{MHz}$ ) compound $7 \mathbf{c}$
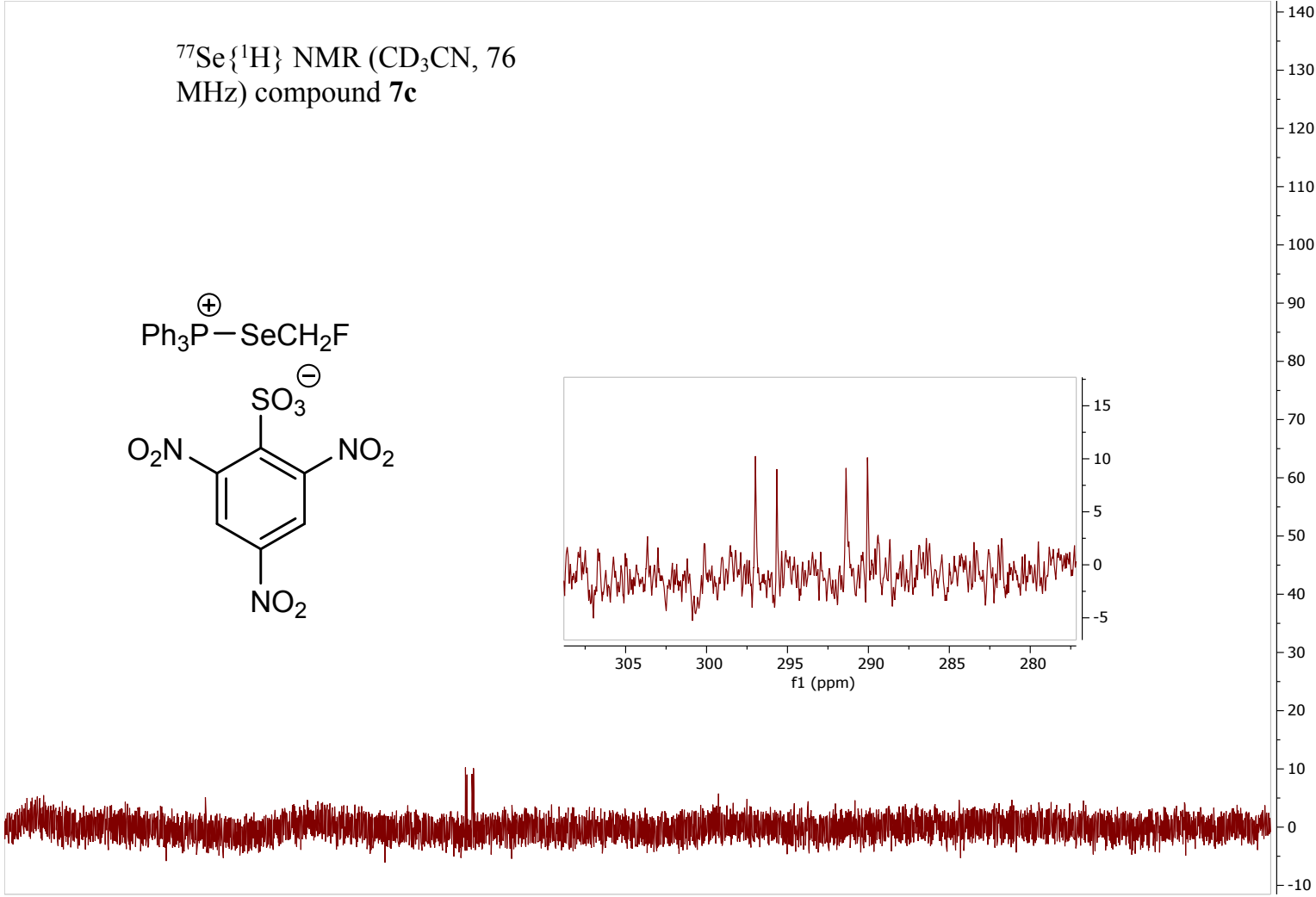

$\begin{array}{llllllllll}650 & 600 & 550 & 500 & 450 & 400 & 350 & 300 & 250 & 200 \\ \mathrm{f} 1(\mathrm{ppm}) & 150 & 100\end{array}$ 
1.13 4-(4-(Dimethylamino) benzoyl) -N- (fluoromethyl) - N,N - dimethyl benzen aminium 2,4,6-trinitrobenzene sulfonate $(\mathbf{8})$

${ }^{1} \mathrm{H} \mathrm{NMR}\left(\mathrm{CD}_{3} \mathrm{CN}, 400\right.$

$\mathrm{MHz}$ ), compound 8

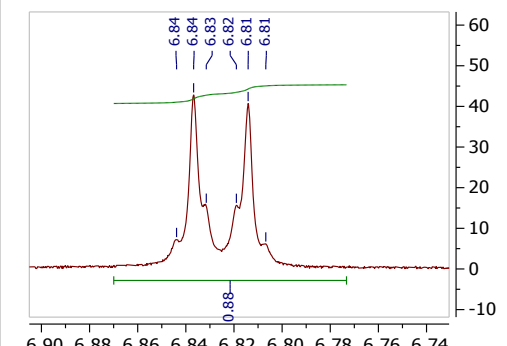

$\begin{array}{lllllllllll}6.90 & 6.88 & 6.86 & 6.84 & 6.82 & 6.80 & 6.78 & 6.76 & 6.74\end{array}$

f1 (ppm)
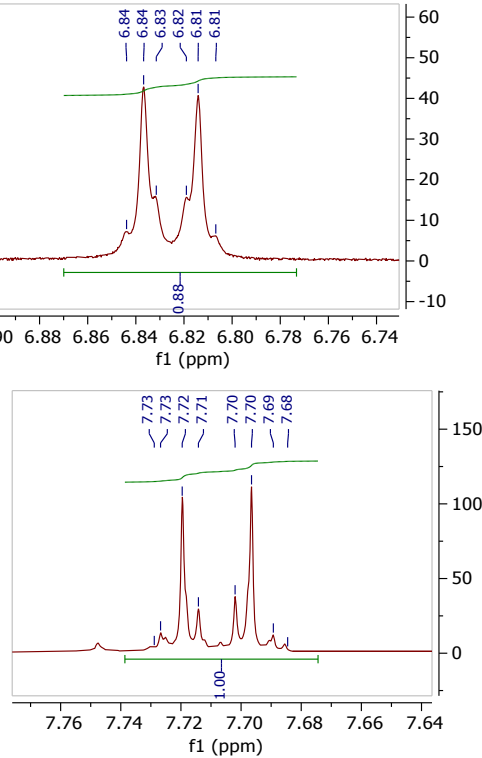

[

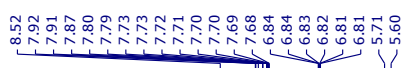

\section{.}

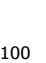

(1)

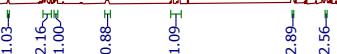

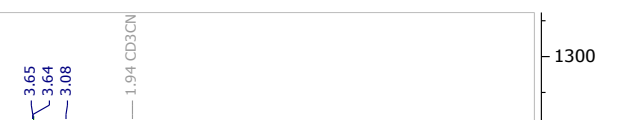

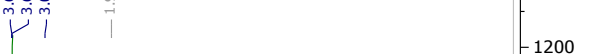<smiles>CN(C)c1ccc(C(=O)c2ccc(N(C)C)cc2)cc1</smiles>

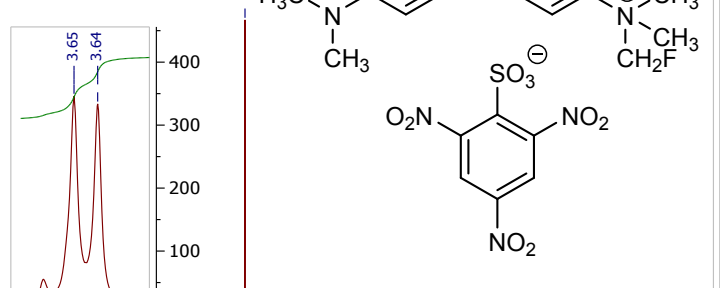

$\begin{array}{llllllllllll}18 & 17 & 16 & 15 & 14 & 13 & 12 & 11 & 10 & 9 & \begin{array}{r}8 \\ \mathrm{f} 1(\mathrm{ppm})\end{array}\end{array}$

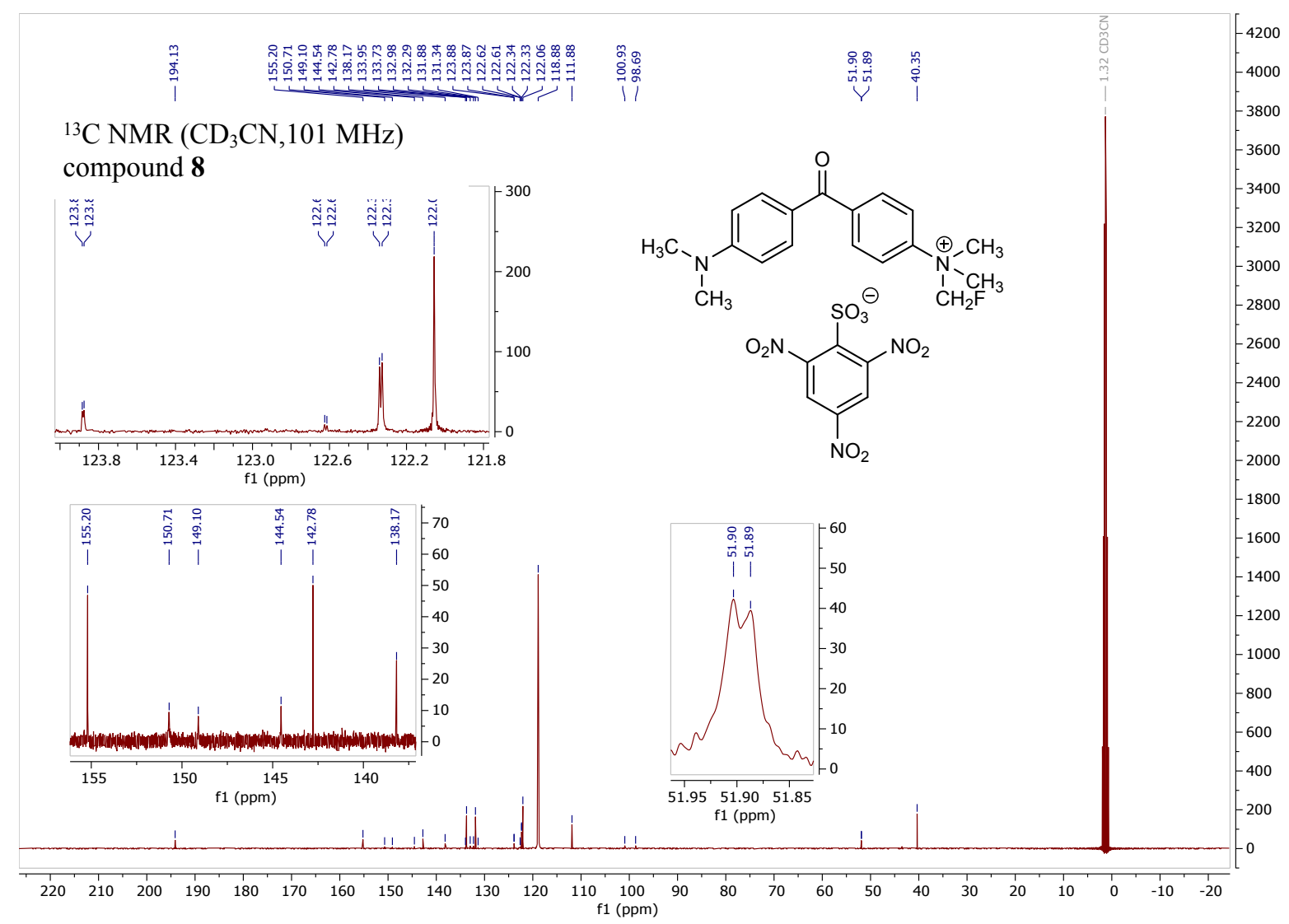


${ }^{19} \mathrm{~F}\left\{{ }^{1} \mathrm{H}\right\}$ NMR $\left(\mathrm{CD}_{3} \mathrm{CN}, 376\right.$

$\mathrm{MHz}$ ) compound 8<smiles></smiles>
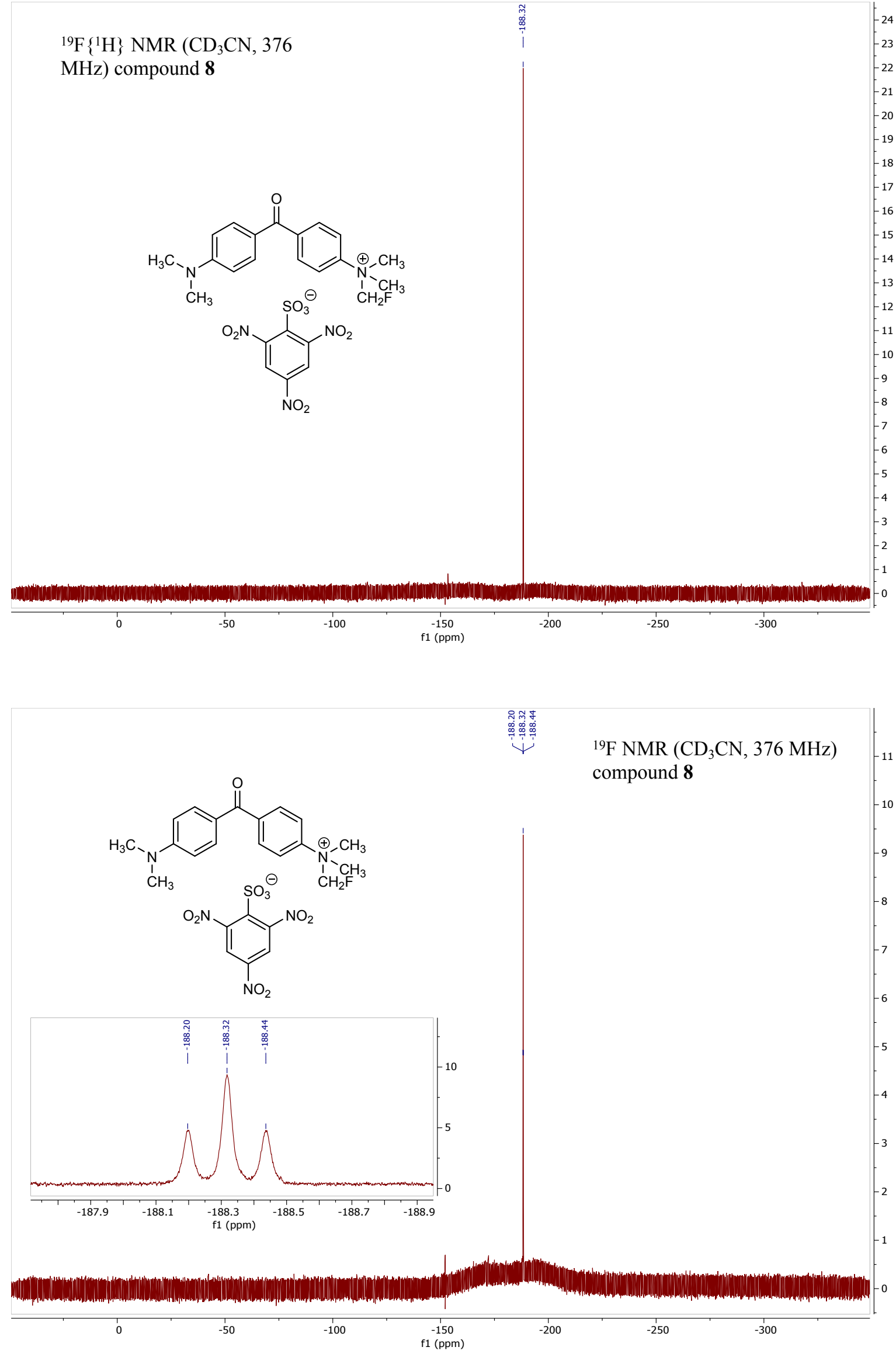

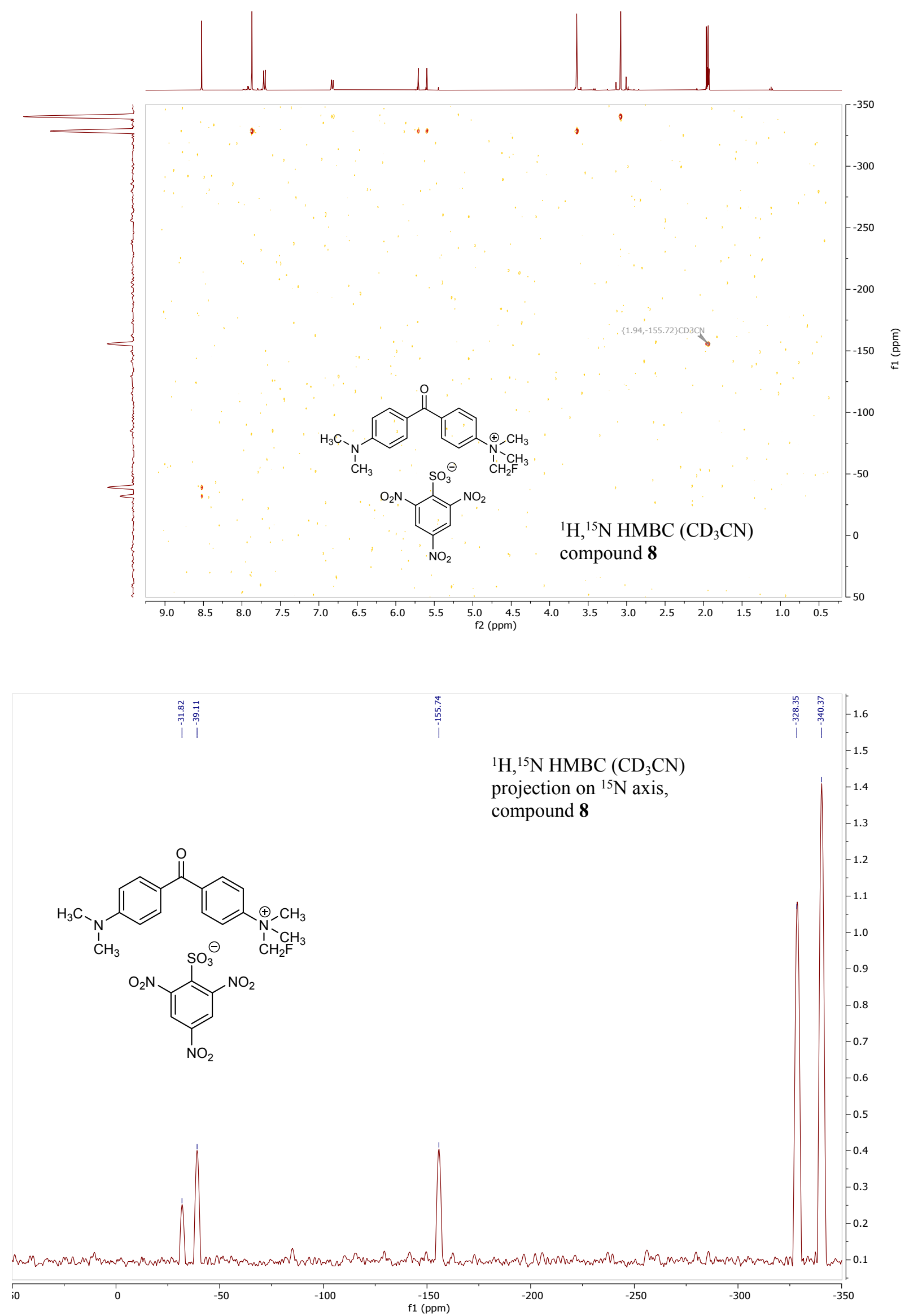
1.14 1-(Fluoromethyl)-2-picolinoylpyridin-1-ium 2,4,6-trinitrobenzenesulfonate (9)
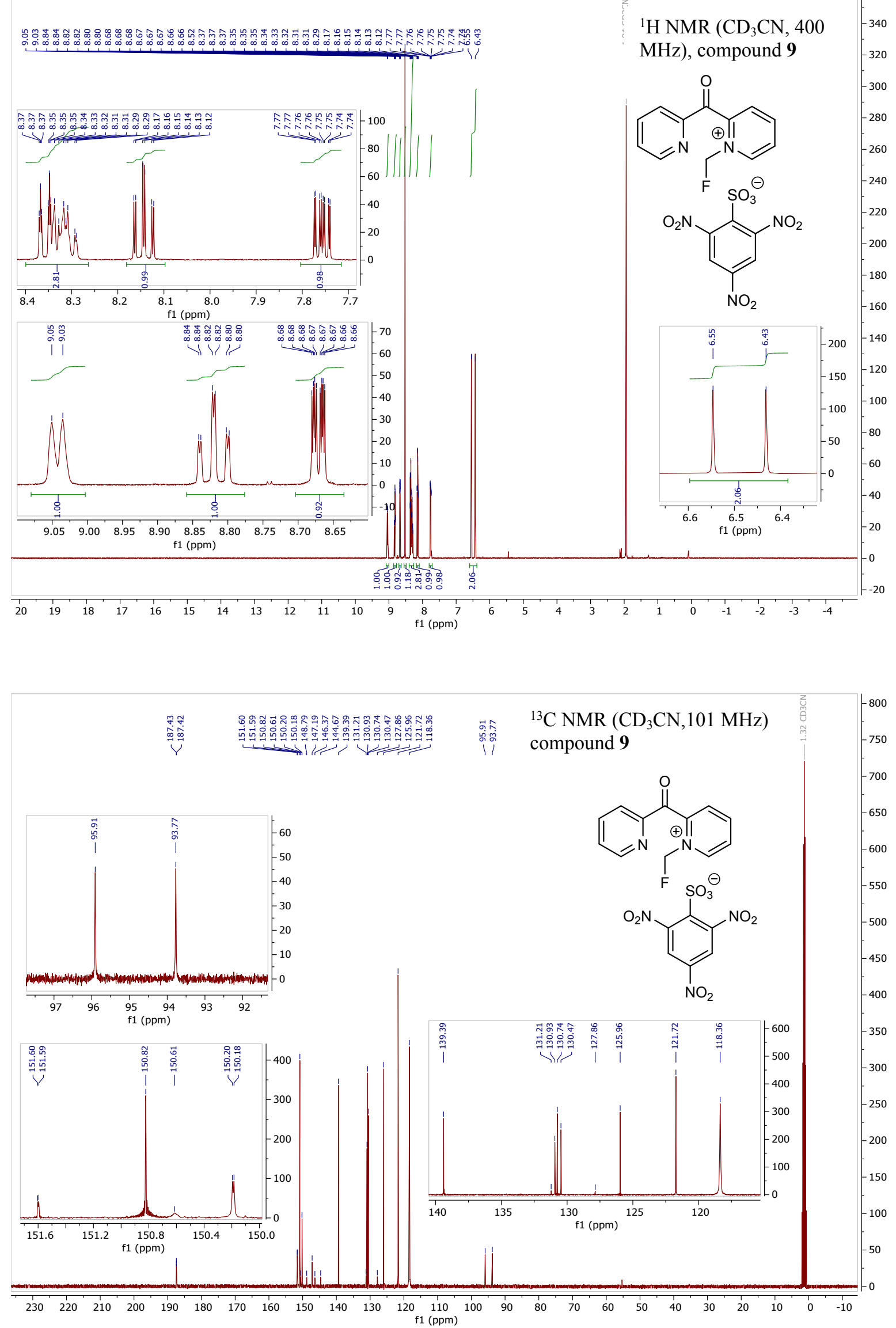

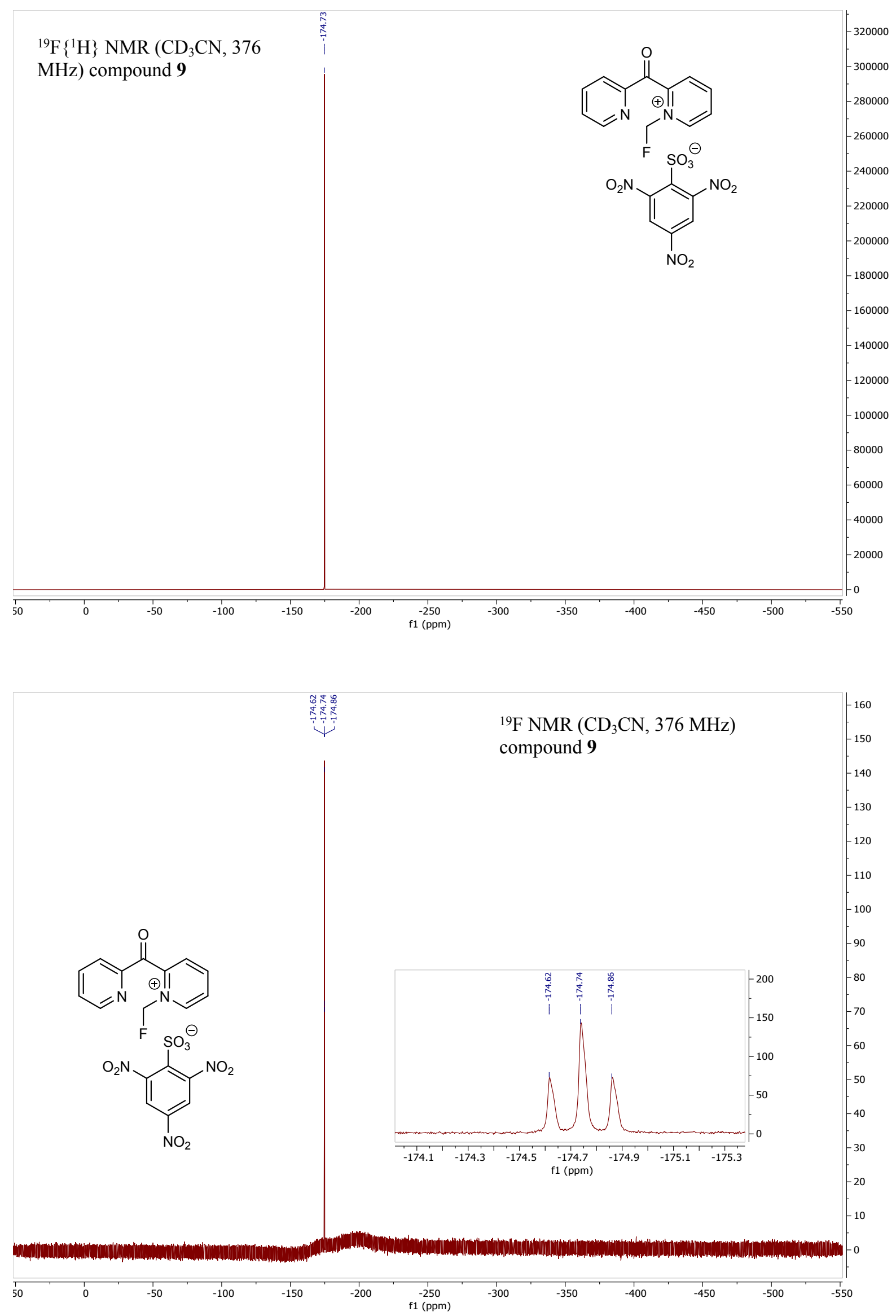

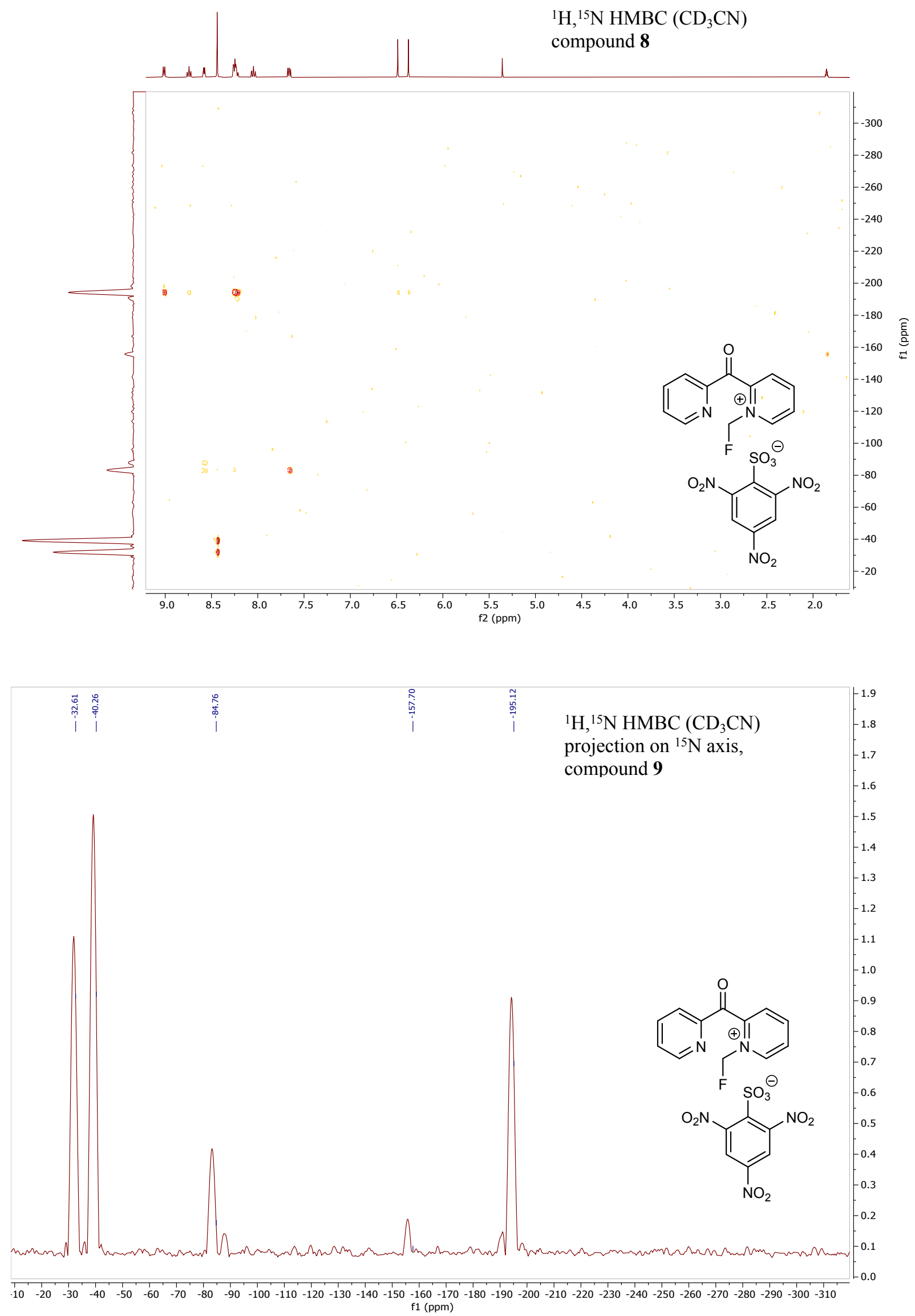


\section{Single Crystal X-Ray Diffraction Studies}

Single crystals of the compounds, suitable for X-ray diffraction, were obtained by slow evaporation of acetonitrile solution. The crystals were introduced into perfluorinated oil and a suitable single crystal was carefully mounted on the top of a thin glass wire. Data collection was performed with an Oxford Xcalibur 3 diffractometer equipped with a Spellman generator $(50 \mathrm{kV}, 40 \mathrm{~mA})$ and a Kappa CCD detector, operating with Mo-K $\mathrm{K}_{\alpha}$ radiation $(\lambda=0.71071 \AA \AA)$.

Data collection and data reduction were performed with the CrysAlisPro software. ${ }^{\text {a) }}$ Absorption correction using the multiscan method ${ }^{\text {a) }}$ was applied. The structures were solved with SHELXS-97, b) refined with SHELXL-97c) and finally checked using PLATON.d)

CCDC-2025108 - 2025118 contain supplementary crystallographic data for the compounds. These data can be obtained free of charge from The Cambridge Crystallographic Data Centre via www.ccdc.cam.ac.uk/data request/cif. 


\subsection{Compound 3}

Table S1. Details for X-ray data collection and structure refinement for compound 3.

\begin{tabular}{|c|c|}
\hline & 3 \\
\hline $\mathrm{CCDC}$ & 2025110 \\
\hline Empirical formula & $\mathrm{C}_{6} \mathrm{H}_{6} \mathrm{AgN}_{3} \mathrm{O}_{11} \mathrm{~S}$ \\
\hline Formula mass & 436.07 \\
\hline $\mathrm{T}[\mathrm{K}]$ & $143(2)$ \\
\hline Crystal size $[\mathrm{mm}]$ & $0.25 \times 0.15 \times 0.04$ \\
\hline Crystal description & colorless platelet \\
\hline Crystal system & triclinic \\
\hline Space group & $P-1$ \\
\hline 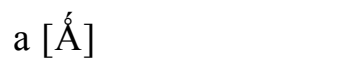 & $8.0870(7)$ \\
\hline $\mathrm{b}[\AA ̊ d]$ & $8.1598(6)$ \\
\hline c [Á] & $10.4594(7)$ \\
\hline$\alpha\left[^{\circ}\right]$ & $75.109(6)$ \\
\hline$\beta\left[^{\circ}\right]$ & $75.218(7)$ \\
\hline$\gamma\left[^{\circ}\right]$ & $67.201(7)$ \\
\hline $\mathrm{V}\left[\AA^{3}\right]$ & $605.46(9)$ \\
\hline$Z$ & 2 \\
\hline$\rho_{\text {calcd. }}\left[\mathrm{g} \mathrm{cm}^{-3}\right]$ & 2.392 \\
\hline$\mu\left[\mathrm{mm}^{-1}\right]$ & 1.910 \\
\hline$F(000)$ & 428 \\
\hline$\Theta$ range $\left[^{\circ}\right]$ & $4.27-25.24$ \\
\hline \multirow[t]{3}{*}{ Index ranges } & $-8 \leq h \leq 10$ \\
\hline & $-10 \leq k \leq 10$ \\
\hline & $-13 \leq l \leq 13$ \\
\hline Reflns. collected & 5369 \\
\hline Reflns. obsd. & 2604 \\
\hline Reflns. unique & $\begin{array}{l}2976 \\
\left(\mathrm{R}_{\mathrm{int}}=0.0237\right)\end{array}$ \\
\hline$R_{1}, w R_{2}(2 \sigma$ data $)$ & $0.0328,0.0658$ \\
\hline$R_{1}, w R_{2}$ (all data) & $0.0392,0.0709$ \\
\hline GOOF on $F^{2}$ & 1.048 \\
\hline Peak/hole $\left[\mathrm{e} \AA^{-3}\right]$ & $1.733 /-1.186$ \\
\hline
\end{tabular}




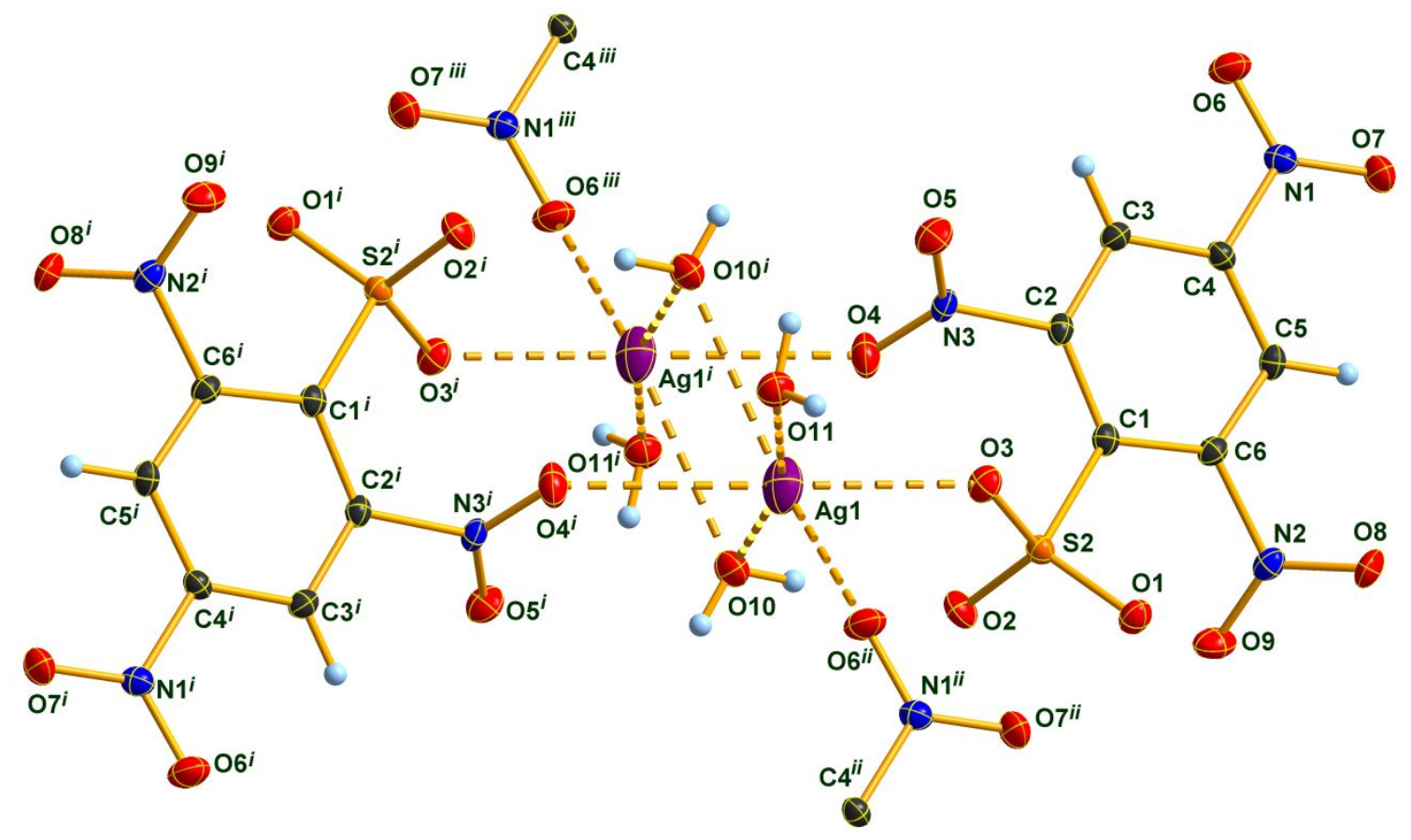

Figure S1. Molecular structure of compound 3 in the crystal. DIAMOND ${ }^{\mathrm{e})}$ representation; thermal ellipsoids are drawn at $50 \%$ probability level. Symmetry codes: $i$ : $-\mathrm{x},-\mathrm{y}, 2-\mathrm{z} ; i i$ : $\mathrm{x}, \mathrm{y}$, 1+z; iii: $-\mathrm{x},-\mathrm{y}, 1-\mathrm{z}$.

Table S2. Selected bond lengths $(\AA ̊)$ of compound 3.

\begin{tabular}{ll|ll}
$\mathrm{Ag} 1-\mathrm{O} 11$ & $2.238(2)$ & $\mathrm{N} 1-\mathrm{O} 6$ & $1.222(3)$ \\
$\mathrm{Ag} 1-\mathrm{O} 10$ & $2.276(2)$ & $\mathrm{N} 1-\mathrm{C} 4$ & $1.463(3)$ \\
$\mathrm{Ag} 1-\mathrm{O} 3$ & $2.532(2)$ & $\mathrm{N} 2-\mathrm{C} 6$ & $1.479(3)$ \\
$\mathrm{O} 1-\mathrm{S} 2$ & $1.442(2)$ & $\mathrm{N} 3-\mathrm{O} 5$ & $1.212(3)$ \\
$\mathrm{S} 2-\mathrm{O} 2$ & $1.445(2)$ & $\mathrm{N} 3-\mathrm{O} 4$ & $1.228(3)$ \\
$\mathrm{S} 2-\mathrm{O} 3$ & $1.456(2)$ & $\mathrm{N} 3-\mathrm{C} 2$ & $1.477(3)$ \\
$\mathrm{S} 2-\mathrm{C} 1$ & $1.806(3)$ & $\mathrm{C} 1-\mathrm{C} 2$ & $1.395(4)$ \\
$\mathrm{O} 9-\mathrm{N} 2$ & $1.213(3)$ & $\mathrm{C} 1-\mathrm{C} 6$ & $1.399(4)$ \\
$\mathrm{O} 7-\mathrm{N} 1$ & $1.228(3)$ & $\mathrm{C} 2-\mathrm{C} 3$ & $1.379(4)$ \\
$\mathrm{O} 8-\mathrm{N} 2$ & $1.227(3)$ & $\mathrm{C} 3-\mathrm{C} 4$ & $1.377(4)$ \\
$\mathrm{C} 5-\mathrm{C} 6$ & $1.378(4)$ & $\mathrm{C} 4-\mathrm{C} 5$ & $1.379(4)$
\end{tabular}

Table S3. Selected bond angles $\left(^{\circ}\right)$ of compound 3 .

$$
\begin{array}{cc|cc}
\mathrm{O} 11-\mathrm{Ag} 1-\mathrm{O} 10 & 162.3(1) & \mathrm{O} 5-\mathrm{N} 3-\mathrm{O} 4 & 125.5(2) \\
\mathrm{O} 11-\mathrm{Ag} 1-\mathrm{O} 3 & 110.1(1) & \mathrm{O} 5-\mathrm{N} 3-\mathrm{C} 2 & 118.5(2) \\
\text { S34 } &
\end{array}
$$




\begin{tabular}{ll|ll}
$\mathrm{O} 10-\mathrm{Ag} 1-\mathrm{O} 3$ & $86.2(1)$ & $\mathrm{O} 4-\mathrm{N} 3-\mathrm{C} 2$ & $115.8(2)$ \\
$\mathrm{O} 1-\mathrm{S} 2-\mathrm{O} 2$ & $115.3(1)$ & $\mathrm{C} 2-\mathrm{C} 1-\mathrm{C} 6$ & $114.5(2)$ \\
$\mathrm{O} 1-\mathrm{S} 2-\mathrm{O} 3$ & $112.9(1)$ & $\mathrm{C} 2-\mathrm{C} 1-\mathrm{S} 2$ & $121.1(2)$ \\
$\mathrm{O} 2-\mathrm{S} 2-\mathrm{O} 3$ & $113.4(1)$ & $\mathrm{C} 6-\mathrm{C} 1-\mathrm{S} 2$ & $123.7(2)$ \\
$\mathrm{O} 1-\mathrm{S} 2-\mathrm{C} 1$ & $104.4(1)$ & $\mathrm{C} 3-\mathrm{C} 2-\mathrm{C} 1$ & $124.3(2)$ \\
$\mathrm{O} 2-\mathrm{S} 2-\mathrm{C} 1$ & $106.7(1)$ & $\mathrm{C} 3-\mathrm{C} 2-\mathrm{N} 3$ & $115.1(2)$ \\
$\mathrm{O} 3-\mathrm{S} 2-\mathrm{C} 1$ & $102.6(1)$ & $\mathrm{C} 1-\mathrm{C} 2-\mathrm{N} 3$ & $120.6(2)$ \\
$\mathrm{S} 2-\mathrm{O} 3-\mathrm{Ag} 1$ & $132.4(1)$ & $\mathrm{C} 4-\mathrm{C} 3-\mathrm{C} 2$ & $117.1(3)$ \\
$\mathrm{O} 6-\mathrm{N} 1-\mathrm{O} 7$ & $124.1(2)$ & $\mathrm{C} 3-\mathrm{C} 4-\mathrm{C} 5$ & $122.8(3)$ \\
$\mathrm{O} 6-\mathrm{N} 1-\mathrm{C} 4$ & $117.8(2)$ & $\mathrm{C} 3-\mathrm{C} 4-\mathrm{N} 1$ & $118.2(2)$ \\
$\mathrm{O} 7-\mathrm{N} 1-\mathrm{C} 4$ & $118.1(2)$ & $\mathrm{C} 5-\mathrm{C} 4-\mathrm{N} 1$ & $118.9(2)$ \\
$\mathrm{O} 9-\mathrm{N} 2-\mathrm{O} 8$ & $125.5(2)$ & $\mathrm{C} 6-\mathrm{C} 5-\mathrm{C} 4$ & $117.0(2)$ \\
$\mathrm{O} 9-\mathrm{N} 2-\mathrm{C} 6$ & $118.0(2)$ & $\mathrm{C} 5-\mathrm{C} 6-\mathrm{C} 1$ & $124.2(2)$ \\
$\mathrm{O} 8-\mathrm{N} 2-\mathrm{C} 6$ & $116.4(2)$ & $\mathrm{C} 5-\mathrm{C} 6-\mathrm{N} 2$ & $115.3(2)$ \\
$\mathrm{C} 1-\mathrm{C} 6-\mathrm{N} 2$ & $120.4(2)$ & &
\end{tabular}

Table S4. Selected torsion angles $\left(^{\circ}\right)$ of compound 3 .

\begin{tabular}{ll|ll}
$\mathrm{O} 1-\mathrm{S} 2-\mathrm{O} 3-\mathrm{Ag} 1$ & $-119.0(2)$ & $\mathrm{C} 2-\mathrm{C} 3-\mathrm{C} 4-\mathrm{C} 5$ & $0.1(4)$ \\
$\mathrm{O} 2-\mathrm{S} 2-\mathrm{O} 3-\mathrm{Ag} 1$ & $14.6(2)$ & $\mathrm{C} 2-\mathrm{C} 3-\mathrm{C} 4-\mathrm{N} 1$ & $179.6(2)$ \\
$\mathrm{C} 1-\mathrm{S} 2-\mathrm{O} 3-\mathrm{Ag} 1$ & $129.2(2)$ & $\mathrm{O} 6-\mathrm{N} 1-\mathrm{C} 4-\mathrm{C} 3$ & $-5.9(4)$ \\
$\mathrm{O} 1-\mathrm{S} 2-\mathrm{C} 1-\mathrm{C} 2$ & $-145.1(2)$ & $\mathrm{O} 7-\mathrm{N} 1-\mathrm{C} 4-\mathrm{C} 3$ & $173.5(2)$ \\
$\mathrm{O} 2-\mathrm{S} 2-\mathrm{C} 1-\mathrm{C} 2$ & $92.4(2)$ & $\mathrm{O} 6-\mathrm{N} 1-\mathrm{C} 4-\mathrm{C} 5$ & $173.6(2)$ \\
$\mathrm{O} 3-\mathrm{S} 2-\mathrm{C} 1-\mathrm{C} 2$ & $-27.1(2)$ & $\mathrm{O} 7-\mathrm{N} 1-\mathrm{C} 4-\mathrm{C} 5$ & $-7.0(4)$ \\
$\mathrm{O} 1-\mathrm{S} 2-\mathrm{C} 1-\mathrm{C} 6$ & $24.7(3)$ & $\mathrm{C} 3-\mathrm{C} 4-\mathrm{C} 5-\mathrm{C} 6$ & $0.1(4)$ \\
$\mathrm{O} 2-\mathrm{S} 2-\mathrm{C} 1-\mathrm{C} 6$ & $-97.8(2)$ & $\mathrm{N} 1-\mathrm{C} 4-\mathrm{C} 5-\mathrm{C} 6$ & $-179.4(2)$ \\
$\mathrm{O} 3-\mathrm{S} 2-\mathrm{C} 1-\mathrm{C} 6$ & $142.7(2)$ & $\mathrm{C} 4-\mathrm{C} 5-\mathrm{C} 6-\mathrm{C} 1$ & $-1.3(4)$ \\
$\mathrm{C} 6-\mathrm{C} 1-\mathrm{C} 2-\mathrm{C} 3$ & $-1.8(4)$ & $\mathrm{C} 4-\mathrm{C} 5-\mathrm{C} 6-\mathrm{N} 2$ & $178.1(2)$ \\
$\mathrm{S} 2-\mathrm{C} 1-\mathrm{C} 2-\mathrm{C} 3$ & $168.9(2)$ & $\mathrm{C} 2-\mathrm{C} 1-\mathrm{C} 6-\mathrm{C} 5$ & $2.1(4)$ \\
$\mathrm{C} 6-\mathrm{C} 1-\mathrm{C} 2-\mathrm{N} 3$ & $175.5(2)$ & $\mathrm{S} 2-\mathrm{C} 1-\mathrm{C} 6-\mathrm{C} 5$ & $-168.3(2)$ \\
$\mathrm{S} 2-\mathrm{C} 1-\mathrm{C} 2-\mathrm{N} 3$ & $-13.8(3)$ & $\mathrm{C} 2-\mathrm{C} 1-\mathrm{C} 6-\mathrm{N} 2$ & $-177.2(2)$ \\
$\mathrm{O} 5-\mathrm{N} 3-\mathrm{C} 2-\mathrm{C} 3$ & $-61.5(3)$ & $\mathrm{S} 2-\mathrm{C} 1-\mathrm{C} 6-\mathrm{N} 2$ & $12.4(4)$ \\
$\mathrm{O} 4-\mathrm{N} 3-\mathrm{C} 2-\mathrm{C} 3$ & $115.0(3)$ & $\mathrm{O} 9-\mathrm{N} 2-\mathrm{C} 6-\mathrm{C} 5$ & $-126.8(3)$ \\
$\mathrm{O} 5-\mathrm{N} 3-\mathrm{C} 2-\mathrm{C} 1$ & $120.9(3)$ & $\mathrm{O} 8-\mathrm{N} 2-\mathrm{C} 6-\mathrm{C} 5$ & $49.7(3)$ \\
$\mathrm{O} 4-\mathrm{N} 3-\mathrm{C} 2-\mathrm{C} 1$ & $-62.5(3)$ & $\mathrm{O} 9-\mathrm{N} 2-\mathrm{C} 6-\mathrm{C} 1$ & $52.6(4)$ \\
$\mathrm{C} 1-\mathrm{C} 2-\mathrm{C} 3-\mathrm{C} 4$ & $0.8(4)$ & $\mathrm{O} 8-\mathrm{N} 2-\mathrm{C} 6-\mathrm{C} 1$ & $-130.9(3)$ \\
$\mathrm{N} 3-\mathrm{C} 2-\mathrm{C} 3-\mathrm{C} 4$ & $-176.7(2)$ & &
\end{tabular}

2.2 Compound 4 
Table S5. Details for X-ray data collection and structure refinement for compound 4.

\begin{tabular}{|c|c|}
\hline & 4 \\
\hline $\mathrm{CCDC}$ & 2025114 \\
\hline Empirical formula & $\mathrm{C}_{7} \mathrm{H}_{4} \mathrm{FN}_{3} \mathrm{O}_{9} \mathrm{~S}$ \\
\hline Formula mass & 325.19 \\
\hline $\mathrm{T}[\mathrm{K}]$ & $143(2)$ \\
\hline Crystal size $[\mathrm{mm}]$ & $0.35 \times 0.20 \times 0.02$ \\
\hline Crystal description & colorless platelet \\
\hline Crystal system & orthorhombic \\
\hline Space group & Pca21 \\
\hline $\mathrm{a}[\AA \hat{́}]$ & $10.4441(3)$ \\
\hline $\mathrm{b}[\AA \hat{A}]$ & $7.0497(2)$ \\
\hline $\mathrm{c}[\AA \hat{́}]$ & $31.5597(10)$ \\
\hline$\alpha\left[^{\circ}\right]$ & 90.0 \\
\hline$\beta\left[^{\circ}\right]$ & 90.0 \\
\hline$\gamma\left[^{\circ}\right]$ & 90.0 \\
\hline $\mathrm{V}\left[\AA^{3}\right]$ & $2323.67(12)$ \\
\hline $\mathrm{Z}$ & 8 \\
\hline$\rho_{\text {calcd. }}\left[\mathrm{g} \mathrm{cm}^{-3}\right]$ & 1.859 \\
\hline$\mu\left[\mathrm{mm}^{-1}\right]$ & 0.350 \\
\hline$F(000)$ & 1312 \\
\hline$\Theta$ range $\left[{ }^{\circ}\right]$ & $4.34-25.24$ \\
\hline \multirow[t]{3}{*}{ Index ranges } & $-14 \leq h \leq 14$ \\
\hline & $-10 \leq k \leq 9$ \\
\hline & $-45 \leq l \leq 44$ \\
\hline Reflns. collected & 23116 \\
\hline Reflns. obsd. & 5890 \\
\hline Reflns. unique & $\begin{array}{l}6869 \\
\left(R_{\text {int }}=0.0406\right)\end{array}$ \\
\hline$R_{1}, w R_{2}(2 \sigma$ data $)$ & $0.0399,0.0878$ \\
\hline$R_{1}, w R_{2}$ (all data) & $0.0510,0.0955$ \\
\hline GOOF on $F^{2}$ & 1.047 \\
\hline Peak/hole [e $\left.\AA^{-3}\right]$ & $0.591 /-0.419$ \\
\hline
\end{tabular}



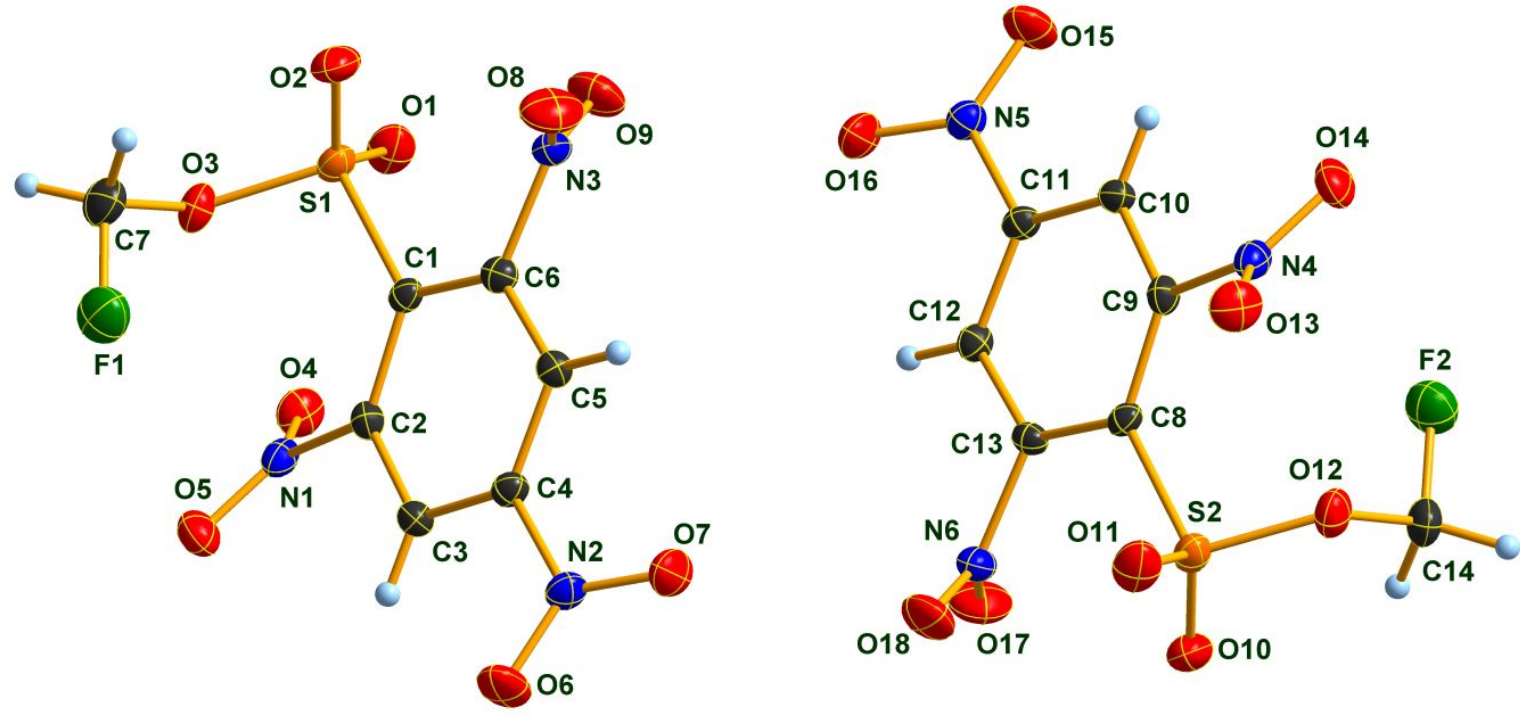

Figure S2. Molecular structure of compound 4 in the crystal. DIAMOND ${ }^{\mathrm{e})}$ representation; thermal ellipsoids are drawn at $50 \%$ probability level.

Table S6. Selected bond lengths $(\AA)$ of compound 4.

\begin{tabular}{ll|ll}
$\mathrm{S} 1-\mathrm{O} 2$ & $1.413(3)$ & $\mathrm{N} 4-\mathrm{O} 13$ & $1.219(4)$ \\
$\mathrm{S} 1-\mathrm{O} 1$ & $1.414(3)$ & $\mathrm{N} 4-\mathrm{O} 14$ & $1.224(4)$ \\
$\mathrm{S} 1-\mathrm{O} 3$ & $1.571(3)$ & $\mathrm{N} 4-\mathrm{C} 9$ & $1.475(4)$ \\
$\mathrm{S} 1-\mathrm{C} 1$ & $1.793(3)$ & $\mathrm{C} 4-\mathrm{C} 5$ & $1.377(4)$ \\
$\mathrm{F} 1-\mathrm{C} 7$ & $1.355(5)$ & $\mathrm{N} 5-\mathrm{O} 15$ & $1.212(4)$ \\
$\mathrm{N} 3-\mathrm{O} 8$ & $1.216(4)$ & $\mathrm{N} 5-\mathrm{O} 16$ & $1.219(5)$ \\
$\mathrm{N} 3-\mathrm{O} 9$ & $1.217(4)$ & $\mathrm{N} 5-\mathrm{C} 11$ & $1.473(5)$ \\
$\mathrm{N} 3-\mathrm{C} 6$ & $1.473(4)$ & $\mathrm{C} 5-\mathrm{C} 6$ & $1.370(5)$ \\
$\mathrm{C} 1-\mathrm{C} 6$ & $1.390(5)$ & $\mathrm{N} 6-\mathrm{O} 18$ & $1.213(4)$ \\
$\mathrm{C} 1-\mathrm{C} 2$ & $1.402(4)$ & $\mathrm{N} 6-\mathrm{O} 17$ & $1.223(4)$ \\
$\mathrm{F} 2-\mathrm{C} 14$ & $1.359(5)$ & $\mathrm{N} 6-\mathrm{C} 13$ & $1.473(4)$ \\
$\mathrm{C} 2-\mathrm{C} 3$ & $1.387(5)$ & $\mathrm{C} 8-\mathrm{C} 9$ & $1.395(4)$ \\
$\mathrm{C} 2-\mathrm{N} 1$ & $1.474(4)$ & $\mathrm{C} 8-\mathrm{C} 13$ & $1.396(4)$ \\
$\mathrm{S} 2-\mathrm{O} 11$ & $1.412(3)$ & $\mathrm{C} 9-\mathrm{C} 10$ & $1.382(5)$ \\
$\mathrm{S} 2-\mathrm{O} 10$ & $1.418(3)$ & $\mathrm{C} 13-\mathrm{C} 12$ & $1.379(4)$ \\
$\mathrm{S} 2-\mathrm{O} 12$ & $1.572(3)$ & $\mathrm{O} 12-\mathrm{C} 14$ & $1.436(5)$ \\
$\mathrm{S} 2-\mathrm{C} 8$ & $1.796(3)$ & $\mathrm{C} 12-\mathrm{C} 11$ & $1.379(4)$ \\
$\mathrm{N} 2-\mathrm{O} 7$ & $1.218(5)$ & $\mathrm{C} 11-\mathrm{C} 10$ & $1.380(5)$ \\
$\mathrm{N} 2-\mathrm{O} 6$ & $1.219(4)$ & $\mathrm{N} 1-\mathrm{O} 5$ & $1.213(4)$
\end{tabular}




$$
\begin{array}{ll|ll}
\mathrm{N} 2-\mathrm{C} 4 & 1.471(5) & \mathrm{N} 1-\mathrm{O} 4 & 1.222(4) \\
\mathrm{O} 3-\mathrm{C} 7 & 1.438(5) & \mathrm{C} 3-\mathrm{C} 4 & 1.383(5)
\end{array}
$$

Table S7. Selected bond angles $\left(^{\circ}\right)$ of compound 4.

$$
\begin{array}{ll|ll}
\mathrm{O} 2-\mathrm{S} 1-\mathrm{O} 1 & 121.5(2) & \mathrm{C} 6-\mathrm{C} 5-\mathrm{C} 4 & 117.7(3) \\
\mathrm{O} 2-\mathrm{S} 1-\mathrm{O} 3 & 109.3(2) & \mathrm{C} 5-\mathrm{C} 6-\mathrm{C} 1 & 123.3(3) \\
\mathrm{O} 1-\mathrm{S} 1-\mathrm{O} 3 & 105.6(2) & \mathrm{C} 5-\mathrm{C} 6-\mathrm{N} 3 & 114.7(3) \\
\mathrm{O} 2-\mathrm{S} 1-\mathrm{C} 1 & 106.1(2) & \mathrm{C} 1-\mathrm{C} 6-\mathrm{N} 3 & 121.9(3) \\
\mathrm{O} 1-\mathrm{S} 1-\mathrm{C} 1 & 109.6(2) & \mathrm{O} 18-\mathrm{N} 6-\mathrm{O} 17 & 126.2(3) \\
\mathrm{O} 3-\mathrm{S} 1-\mathrm{C} 1 & 103.3(1) & \mathrm{O} 18-\mathrm{N} 6-\mathrm{C} 13 & 116.9(3) \\
\mathrm{O} 8-\mathrm{N} 3-\mathrm{O} 9 & 126.1(3) & \mathrm{O} 17-\mathrm{N} 6-\mathrm{C} 13 & 116.9(3) \\
\mathrm{O} 8-\mathrm{N} 3-\mathrm{C} 6 & 117.3(3) & \mathrm{F} 1-\mathrm{C} 7-\mathrm{O} 3 & 107.8(3) \\
\mathrm{O} 9-\mathrm{N} 3-\mathrm{C} 6 & 116.5(3) & \mathrm{C} 9-\mathrm{C} 8-\mathrm{C} 13 & 115.9(3) \\
\mathrm{C} 6-\mathrm{C} 1-\mathrm{C} 2 & 116.2(3) & \mathrm{C} 9-\mathrm{C} 8-\mathrm{S} 2 & 125.0(2) \\
\mathrm{C} 6-\mathrm{C} 1-\mathrm{S} 1 & 119.2(2) & \mathrm{C} 13-\mathrm{C} 8-\mathrm{S} 2 & 119.0(2) \\
\mathrm{C} 2-\mathrm{C} 1-\mathrm{S} 1 & 124.5(2) & \mathrm{C} 10-\mathrm{C} 9-\mathrm{C} 8 & 123.2(3) \\
\mathrm{C} 3-\mathrm{C} 2-\mathrm{C} 1 & 122.7(3) & \mathrm{C} 10-\mathrm{C} 9-\mathrm{N} 4 & 115.2(3) \\
\mathrm{C} 3-\mathrm{C} 2-\mathrm{N} 1 & 115.3(3) & \mathrm{C} 8-\mathrm{C} 9-\mathrm{N} 4 & 121.5(3) \\
\mathrm{C} 1-\mathrm{C} 2-\mathrm{N} 1 & 121.8(3) & \mathrm{C} 12-\mathrm{C} 13-\mathrm{C} 8 & 123.4(3) \\
\mathrm{O} 11-\mathrm{S} 2-\mathrm{O} 10 & 121.3(2) & \mathrm{C} 12-\mathrm{C} 13-\mathrm{N} 6 & 114.7(3) \\
\mathrm{O} 11-\mathrm{S} 2-\mathrm{O} 12 & 106.2(2) & \mathrm{C} 8-\mathrm{C} 13-\mathrm{N} 6 & 121.9(3) \\
\mathrm{O} 10-\mathrm{S} 2-\mathrm{O} 12 & 108.9(2) & \mathrm{C} 14-\mathrm{O} 12-\mathrm{S} 2 & 120.3(3) \\
\mathrm{O} 11-\mathrm{S} 2-\mathrm{C} 8 & 109.5(2) & \mathrm{C} 13-\mathrm{C} 12-\mathrm{C} 11 & 117.1(3) \\
\mathrm{O} 10-\mathrm{S} 2-\mathrm{C} 8 & 105.9(2) & \mathrm{C} 12-\mathrm{C} 11-\mathrm{C} 10 & 123.2(3) \\
\mathrm{O} 12-\mathrm{S} 2-\mathrm{C} 8 & 103.8(1) & \mathrm{C} 12-\mathrm{C} 11-\mathrm{N} 5 & 117.7(3) \\
\mathrm{O} 7-\mathrm{N} 2-\mathrm{O} 6 & 125.0(3) & \mathrm{C} 10-\mathrm{C} 11-\mathrm{N} 5 & 119.1(3) \\
\mathrm{O} 7-\mathrm{N} 2-\mathrm{C} 4 & 117.3(3) & \mathrm{C} 11-\mathrm{C} 10-\mathrm{C} 9 & 117.1(3) \\
\mathrm{O} 6-\mathrm{N} 2-\mathrm{C} 4 & 117.7(4) & \mathrm{F} 2-\mathrm{C} 14-\mathrm{O} 12 & 107.3(3) \\
\mathrm{C} 7-\mathrm{O} 3-\mathrm{S} 1 & 120.5(3) & \mathrm{C} 5-\mathrm{C} 4-\mathrm{C} 3 & 123.0(3) \\
\mathrm{O} 5-\mathrm{N} 1-\mathrm{O} 4 & 126.1(3) & \mathrm{C} 5-\mathrm{C} 4-\mathrm{N} 2 & 118.1(3) \\
\mathrm{O} 5-\mathrm{N} 1-\mathrm{C} 2 & 117.4(3) & \mathrm{C} 3-\mathrm{C} 4-\mathrm{N} 2 & 118.9(3) \\
\mathrm{O} 4-\mathrm{N} 1-\mathrm{C} 2 & 116.4(3) & \mathrm{O} 15-\mathrm{N} 5-\mathrm{O} 16 & 125.3(3) \\
\mathrm{C} 4-\mathrm{C} 3-\mathrm{C} 2 & 117.0(3) & \mathrm{O} 15-\mathrm{N} 5-\mathrm{C} 11 & 117.6(4) \\
\mathrm{O} 13-\mathrm{N} 4-\mathrm{O} 14 & 125.5(3) & \mathrm{O} 16-\mathrm{N} 5-\mathrm{C} 11 & 117.1(3) \\
\mathrm{O} 13-\mathrm{N} 4-\mathrm{C} 9 & 117.0(3) & \mathrm{O} 14-\mathrm{N} 4-\mathrm{C} 9 & 117.4(3)
\end{array}
$$

Table S8. Selected torsion angles $\left(^{\circ}\right)$ of compound 4.

$$
\begin{array}{ll|l}
\mathrm{O} 2 & -\mathrm{S} 1-\mathrm{C} 1-\mathrm{C} 6 \quad-32.8(3) & \mathrm{O} 11-\mathrm{S} 2-\mathrm{C} 8-\mathrm{C} 9 \quad 83.4(3)
\end{array}
$$




\begin{tabular}{|c|c|c|c|}
\hline $1-\mathrm{S} 1-\mathrm{C} 1-\mathrm{C} 6$ & $100.1(3)$ & $\mathrm{O} 10-\mathrm{S} 2-\mathrm{C}$ & $-144.3(3)$ \\
\hline $3-\mathrm{S} 1-\mathrm{C} 1-\mathrm{C} 6$ & $-147.8(2)$ & $12-\mathrm{S} 2-\mathrm{C} 8-\mathrm{C} 9$ & $-29.7(3)$ \\
\hline$-\mathrm{S} 1-\mathrm{C} 1-\mathrm{C} 2$ & $3.0(3)$ & $1-\mathrm{S} 2-\mathrm{C} 8-\mathrm{C} 13$ & $-99.6(3)$ \\
\hline $1-\mathrm{S} 1-\mathrm{C} 1-\mathrm{C} 2$ & -84 & $0-\mathrm{S} 2-\mathrm{C} 8-\mathrm{C} 13$ & $32.8(3)$ \\
\hline $3-\mathrm{S} 1-\mathrm{C} 1-\mathrm{C} 2$ & $28.0(3)$ & $12-\mathrm{S} 2-\mathrm{C} 8-\mathrm{C} 13$ & $147.4(2)$ \\
\hline$-\mathrm{C} 1-\mathrm{C} 2-\mathrm{C} 3$ & $2.4(5)$ & $3-\mathrm{C} 8-\mathrm{C} 9-\mathrm{C} 10$ & -3. \\
\hline$-\mathrm{C} 1-\mathrm{C} 2-\mathrm{C} 3$ & ) & $-\mathrm{C} 8-\mathrm{C} 9-\mathrm{C} 10$ & 3) \\
\hline$-\mathrm{C} 1-\mathrm{C} 2-\mathrm{N} 1$ & & $-\mathrm{N} 4$ & \\
\hline$-\mathrm{C} 1-\mathrm{C} 2-\mathrm{N} 1$ & 11.3 & $2-\mathrm{C} 8-\mathrm{C} 9-\mathrm{N} 4$ & \\
\hline $2-\mathrm{S} 1-\mathrm{O} 3-\mathrm{C} 7$ & -32 . & $13-\mathrm{N} 4-\mathrm{C} 9-\mathrm{C} 10$ & 3) \\
\hline$-\mathrm{S} 1-\mathrm{O} 3-\mathrm{C} 7$ & -16 & $4-\mathrm{N} 4-\mathrm{C} 9-\mathrm{C} 10$ & (4) \\
\hline$-\mathrm{S} 1-\mathrm{O} 3-\mathrm{C} 7$ & $80.4(3)$ & $3-\mathrm{N} 4-\mathrm{C} 9-\mathrm{C} 8$ & -43.9 \\
\hline $3-\mathrm{C} 2-\mathrm{N} 1-\mathrm{O} 5$ & $45.9(4)$ & $14-\mathrm{N} 4-\mathrm{C} 9-\mathrm{C} 8$ & $138.7(3)$ \\
\hline$-\mathrm{C} 2-\mathrm{N} 1-\mathrm{O} 5$ & $-138.6(3)$ & $99-\mathrm{C} 8-\mathrm{C} 13-\mathrm{C} 12$ & $-0.7(5)$ \\
\hline $3-\mathrm{C} 2-\mathrm{N} 1-\mathrm{O} 4$ & $-131.5(3)$ & $\mathrm{S} 2-\mathrm{C} 8-\mathrm{C} 13-\mathrm{C} 12$ & $-178.1(2)$ \\
\hline$-\mathrm{C} 2-\mathrm{N} 1-\mathrm{O} 4$ & 44. & $\mathrm{C} 9-\mathrm{C} 8-\mathrm{C} 13-\mathrm{N} 6$ & $4(3)$ \\
\hline$-\mathrm{C} 2-\mathrm{C} 3-\mathrm{C} 4$ & $-3.7(5)$ & $\mathrm{S} 2-\mathrm{C} 8-\mathrm{C} 13-\mathrm{N} 6$ & $5.2(4)$ \\
\hline$-\mathrm{C} 2-\mathrm{C} 3-\mathrm{C} 4$ & 171. & $18-\mathrm{N} 6-\mathrm{C} 13-\mathrm{C} 12$ & -118 \\
\hline$-\mathrm{C} 4-\mathrm{C} 5$ & 1. & $7-\mathrm{N} 6-\mathrm{C} 13-\mathrm{C} 12$ & 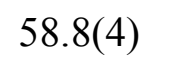 \\
\hline $2-\mathrm{C} 3-\mathrm{C} 4-\mathrm{N} 2$ & $-178.3(3)$ & $\mathrm{O} 18-\mathrm{N} 6-$ & $58.7(5)$ \\
\hline $7-\mathrm{N} 2-\mathrm{C} 4-\mathrm{C} 5$ & $-21.8(5)$ & $\mathrm{O} 17-\mathrm{N} 6-\mathrm{C} 13-\mathrm{C} 8$ & $-124.2(3)$ \\
\hline $5-\mathrm{N} 2-\mathrm{C} 4-\mathrm{C} 5$ & 157.3 & $11-\mathrm{S} 2-\mathrm{O} 12-\mathrm{C} 14$ & $164.2(3)$ \\
\hline $7-\mathrm{N} 2-\mathrm{C} 4-\mathrm{C} 3$ & 158 & $-\mathrm{S} 2-\mathrm{O} 12-\mathrm{C}$ & $32.1(3)$ \\
\hline $6-\mathrm{N} 2-\mathrm{C} 4-\mathrm{C} 3$ & -22 . & $\mathrm{C} 8-\mathrm{S} 2-\mathrm{O} 12-\mathrm{C} 14$ & $4(3)$ \\
\hline$-\mathrm{C} 4-\mathrm{C} 5-\mathrm{C} 6$ & $1.5(5)$ & $\mathrm{C} 8-\mathrm{C} 13-\mathrm{C} 12-\mathrm{C} 11$ & $2.7(5)$ \\
\hline$-\mathrm{C} 4-\mathrm{C} 5-\mathrm{C} 6$ & -178 & $\mathrm{~N} 6-\mathrm{C} 13-\mathrm{C} 12-\mathrm{C} 11$ & 179.7(3) \\
\hline$-\mathrm{C} 5-\mathrm{C} 6-\mathrm{C} 1$ & $-2.9(5)$ & $13-\mathrm{C} 12-\mathrm{C} 11-\mathrm{C} 10$ & $-1.2(5)$ \\
\hline $4-\mathrm{C} 5-\mathrm{C} 6-\mathrm{N} 3$ & $7(3)$ & $\mathrm{C} 13-\mathrm{C} 12-\mathrm{C} 11-\mathrm{I}$ & 178.1(3) \\
\hline $2-\mathrm{C} 1-\mathrm{C} 6-\mathrm{C} 5$ & $1.0(5)$ & $\mathrm{O} 15-\mathrm{N} 5-\mathrm{C} 11-\mathrm{C} 12$ & $-155.5(3)$ \\
\hline$-\mathrm{C} 1-\mathrm{C} 6-\mathrm{C} 5$ & $177.2(3)$ & $16-\mathrm{N} 5-\mathrm{C} 11-\mathrm{C} 12$ & \\
\hline $2-\mathrm{C} 1-\mathrm{C} 6-\mathrm{N} 3$ & $177.6(3)$ & $\mathrm{O} 15-\mathrm{N} 5-\mathrm{C} 11-\mathrm{C} 10$ & $23.9(5)$ \\
\hline $\mathrm{S} 1-\mathrm{C} 1-\mathrm{C} 6-\mathrm{N} 3$ & $-6.2(4)$ & $\mathrm{O} 16-\mathrm{N} 5-\mathrm{C} 11-\mathrm{C} 10$ & $-156.4(3)$ \\
\hline $\mathrm{O} 8-\mathrm{N} 3-\mathrm{C} 6-\mathrm{C} 5$ & $-56.5(4)$ & $\mathrm{C} 12-\mathrm{C} 11-\mathrm{C} 10-\mathrm{C} 9$ & $-2.2(5)$ \\
\hline $9-\mathrm{N} 3-\mathrm{C} 6-\mathrm{C} 5$ & $120.8(3)$ & $\mathrm{N} 5-\mathrm{C} 11-\mathrm{C} 10-\mathrm{C} 9$ & $178.4(3)$ \\
\hline $8-\mathrm{N} 3-\mathrm{C} 6-\mathrm{C} 1$ & $126.7(4)$ & $\mathrm{C} 8-\mathrm{C} 9-\mathrm{C} 10-\mathrm{C} 11$ & $4.4(5)$ \\
\hline $\mathrm{O} 9-\mathrm{N} 3-\mathrm{C} 6-\mathrm{C} 1$ & $-56.0(5)$ & $\mathrm{N} 4-\mathrm{C} 9-\mathrm{C} 10-\mathrm{C} 11$ & -172.2 \\
\hline $1-\mathrm{O} 3-\mathrm{C} 7-\mathrm{F} 1$ & $-92.9(4)$ & $\mathrm{S} 2-\mathrm{O} 12-\mathrm{C} 14-\mathrm{F} 2$ & $95.6(3)$ \\
\hline
\end{tabular}

2.3 Dimethyltrinitroaniline 
Table S9. Details for X-ray data collection and structure refinement for $N, N$-dimethyl-2,4,6trinitroaniline.

\begin{tabular}{|c|c|}
\hline & $\begin{array}{c}N, N \text {-dimethyl-2,4,6- } \\
\text { trinitroaniline }\end{array}$ \\
\hline $\mathrm{CCDC}$ & 2025111 \\
\hline Empirical formula & $\mathrm{C}_{8} \mathrm{H}_{8} \mathrm{~N}_{4} \mathrm{O}_{6}$ \\
\hline Formula mass & 256.18 \\
\hline $\mathrm{T}[\mathrm{K}]$ & $143(2)$ \\
\hline Crystal size [mm] & $0.50 \times 0.40 \times 0.30$ \\
\hline Crystal description & yellow block \\
\hline Crystal system & monoclinic \\
\hline Space group & $P 21 / c$ \\
\hline 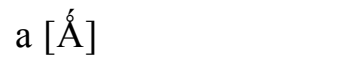 & $8.5353(4)$ \\
\hline $\mathrm{b}[\AA \grave{A}]$ & $16.4216(5)$ \\
\hline $\mathrm{c}[\AA \hat{́}]$ & $8.3436(4)$ \\
\hline$\alpha\left[^{\circ}\right]$ & 90.0 \\
\hline$\beta\left[^{\circ}\right]$ & $118.618(7)$ \\
\hline$\gamma\left[^{\circ}\right]$ & 90.0 \\
\hline $\mathrm{V}\left[\AA^{3}\right]$ & $1026.60(10)$ \\
\hline $\mathrm{Z}$ & 4 \\
\hline$\rho_{\text {calcd. }}\left[\mathrm{g} \mathrm{cm}^{-3}\right]$ & 1.658 \\
\hline$\mu\left[\mathrm{mm}^{-1}\right]$ & 0.144 \\
\hline$F(000)$ & 528 \\
\hline$\Theta$ range $\left[{ }^{\circ}\right]$ & $4.61-25.24$ \\
\hline \multirow[t]{3}{*}{ Index ranges } & $-12 \leq h \leq 12$ \\
\hline & $-23 \leq k \leq 22$ \\
\hline & $-11 \leq l \leq 11$ \\
\hline Reflns. collected & 10637 \\
\hline Reflns. obsd. & 2762 \\
\hline Reflns. unique & $\begin{array}{l}3126 \\
\left(\mathrm{R}_{\mathrm{int}}=0.0173\right)\end{array}$ \\
\hline$R_{1}, w R_{2}(2 \sigma$ data $)$ & $0.0334,0.0943$ \\
\hline$R_{1}, w R_{2}$ (all data) & $0.0384,0.0988$ \\
\hline GOOF on $F^{2}$ & 1.049 \\
\hline Peak/hole $\left[\mathrm{e} \AA^{-3}\right]$ & $0.414 /-0.249$ \\
\hline
\end{tabular}




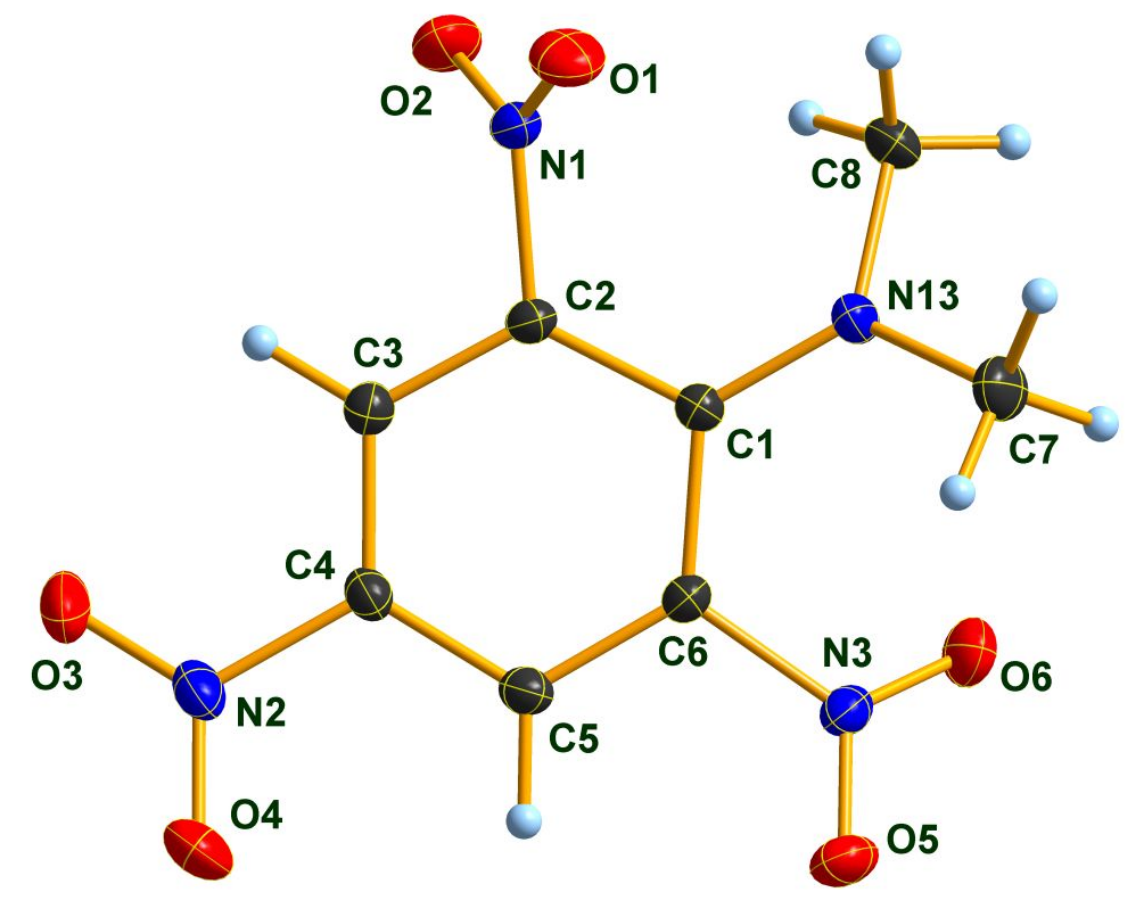

Figure S3. Molecular structure of compound $N, N$-dimethyl-2,4,6-trinitroaniline in the crystal. DIAMOND $^{\mathrm{e})}$ representation; thermal ellipsoids are drawn at $50 \%$ probability level.

Table S10. Selected bond lengths $(\AA)$ of compound $N, N$-dimethyl-2,4,6-trinitroaniline.

\begin{tabular}{ll|ll}
$\mathrm{N} 13-\mathrm{C} 1$ & $1.352(1)$ & $\mathrm{O} 5-\mathrm{N} 3$ & $1.228(1)$ \\
$\mathrm{N} 13-\mathrm{C} 8$ & $1.458(1)$ & $\mathrm{C} 4-\mathrm{C} 3$ & $1.387(1)$ \\
$\mathrm{N} 13-\mathrm{C} 7$ & $1.459(1)$ & $\mathrm{C} 4-\mathrm{N} 2$ & $1.455(1)$ \\
$\mathrm{C} 1-\mathrm{C} 2$ & $1.423(1)$ & $\mathrm{O} 4-\mathrm{N} 2$ & $1.226(1)$ \\
$\mathrm{C} 1-\mathrm{C} 6$ & $1.428(1)$ & $\mathrm{C} 2-\mathrm{C} 3$ & $1.380(1)$ \\
$\mathrm{O} 1-\mathrm{N} 1$ & $1.2240(1)$ & $\mathrm{N} 2-\mathrm{O} 3$ & $1.225(1)$ \\
$\mathrm{N} 1-\mathrm{O} 2$ & $1.222(1)$ & $\mathrm{N} 3-\mathrm{O} 6$ & $1.226(1)$ \\
$\mathrm{N} 1-\mathrm{C} 2$ & $1.471(1)$ & $\mathrm{N} 3-\mathrm{C} 6$ & $1.466(1)$ \\
$\mathrm{C} 5-\mathrm{C} 6$ & $1.382(1)$ & $\mathrm{C} 5-\mathrm{C} 4$ & $1.384(1)$
\end{tabular}

Table S11. Selected bond angles $\left(^{\circ}\right)$ of compound $N, N$-dimethyl-2,4,6-trinitroaniline.

$$
\begin{array}{ll|ll}
\mathrm{C} 1-\mathrm{N} 13-\mathrm{C} 8 & 123.3(1) & \mathrm{C} 3-\mathrm{C} 2-\mathrm{C} 1 & 124.7(1) \\
\mathrm{C} 1-\mathrm{N} 13-\mathrm{C} 7 & 122.8(1) & \mathrm{C} 3-\mathrm{C} 2-\mathrm{N} 1 & 114.3(1) \\
\mathrm{C} 8-\mathrm{N} 13-\mathrm{C} 7 & 113.9(1) & \mathrm{C} 1-\mathrm{C} 2-\mathrm{N} 1 & 120.6(1) \\
\mathrm{N} 13-\mathrm{C} 1-\mathrm{C} 2 & 123.5(1) & \mathrm{O} 3-\mathrm{N} 2-\mathrm{O} 4 & 123.9(1) \\
\mathrm{N} 13-\mathrm{C} 1-\mathrm{C} 6 & 123.7(1) & \mathrm{O} 3-\mathrm{N} 2-\mathrm{C} 4 & 118.1(1)
\end{array}
$$




$$
\begin{array}{ll|ll}
\mathrm{C} 2-\mathrm{C} 1-\mathrm{C} 6 & 112.9(1) & \mathrm{O} 4-\mathrm{N} 2-\mathrm{C} 4 & 118.0(1) \\
\mathrm{O} 2-\mathrm{N} 1-\mathrm{O} 1 & 124.9(1) & \mathrm{C} 2-\mathrm{C} 3-\mathrm{C} 4 & 118.1(1) \\
\mathrm{O} 2-\mathrm{N} 1-\mathrm{C} 2 & 117.3(1) & \mathrm{O} 6-\mathrm{N} 3-\mathrm{O} 5 & 124.3(1) \\
\mathrm{O} 1-\mathrm{N} 1-\mathrm{C} 2 & 117.7(1) & \mathrm{O} 6-\mathrm{N} 3-\mathrm{C} 6 & 117.9(1) \\
\mathrm{C} 6-\mathrm{C} 5-\mathrm{C} 4 & 118.5(1) & \mathrm{O} 5-\mathrm{N} 3-\mathrm{C} 6 & 117.7(1) \\
\mathrm{C} 5-\mathrm{C} 4-\mathrm{C} 3 & 121.5(1) & \mathrm{C} 5-\mathrm{C} 6-\mathrm{C} 1 & 124.0(1) \\
\mathrm{C} 5-\mathrm{C} 4-\mathrm{N} 2 & 119.0(1) & \mathrm{C} 5-\mathrm{C} 6-\mathrm{N} 3 & 114.4(1) \\
\mathrm{C} 3-\mathrm{C} 4-\mathrm{N} 2 & 119.5(1) & \mathrm{C} 1-\mathrm{C} 6-\mathrm{N} 3 & 121.2(1)
\end{array}
$$

Table S12. Selected torsion angles $\left(^{\circ}\right)$ of compound $N, N$-dimethyl-2,4,6-trinitroaniline.

\begin{tabular}{ll|ll}
$\mathrm{C} 8-\mathrm{N} 13-\mathrm{C} 1-\mathrm{C} 2$ & $-32.3(1)$ & $\mathrm{C} 5-\mathrm{C} 4-\mathrm{N} 2-\mathrm{O} 4$ & $12.6(1)$ \\
$\mathrm{C} 7-\mathrm{N} 13-\mathrm{C} 1-\mathrm{C} 2$ & $146.3(1)$ & $\mathrm{C} 3-\mathrm{C} 4-\mathrm{N} 2-\mathrm{O} 4$ & $-167.1(1)$ \\
$\mathrm{C} 8-\mathrm{N} 13-\mathrm{C} 1-\mathrm{C} 6$ & $148.5(1)$ & $\mathrm{C} 1-\mathrm{C} 2-\mathrm{C} 3-\mathrm{C} 4$ & $2.1(1)$ \\
$\mathrm{C} 7-\mathrm{N} 13-\mathrm{C} 1-\mathrm{C} 6$ & $-32.9(1)$ & $\mathrm{N} 1-\mathrm{C} 2-\mathrm{C} 3-\mathrm{C} 4$ & $-170.6(1)$ \\
$\mathrm{C} 6-\mathrm{C} 5-\mathrm{C} 4-\mathrm{C} 3$ & $-0.3(1)$ & $\mathrm{C} 5-\mathrm{C} 4-\mathrm{C} 3-\mathrm{C} 2$ & $-3.3(1)$ \\
$\mathrm{C} 6-\mathrm{C} 5-\mathrm{C} 4-\mathrm{N} 2$ & $-178.0(1)$ & $\mathrm{N} 2-\mathrm{C} 4-\mathrm{C} 3-\mathrm{C} 2$ & $176.4(1)$ \\
$\mathrm{N} 13-\mathrm{C} 1-\mathrm{C} 2-\mathrm{C} 3$ & $-177.0(1)$ & $\mathrm{C} 4-\mathrm{C} 5-\mathrm{C} 6-\mathrm{C} 1$ & $5.3(1)$ \\
$\mathrm{C} 6-\mathrm{C} 1-\mathrm{C} 2-\mathrm{C} 3$ & $2.3(1)$ & $\mathrm{C} 4-\mathrm{C} 5-\mathrm{C} 6-\mathrm{N} 3$ & $-166.9(1)$ \\
$\mathrm{N} 13-\mathrm{C} 1-\mathrm{C} 2-\mathrm{N} 1$ & $-4.8(1)$ & $\mathrm{N} 13-\mathrm{C} 1-\mathrm{C} 6-\mathrm{C} 5$ & $173.2(1)$ \\
$\mathrm{C} 6-\mathrm{C} 1-\mathrm{C} 2-\mathrm{N} 1$ & $174.5(1)$ & $\mathrm{C} 2-\mathrm{C} 1-\mathrm{C} 6-\mathrm{C} 5$ & $-6.1(1)$ \\
$\mathrm{O} 2-\mathrm{N} 1-\mathrm{C} 2-\mathrm{C} 3$ & $-60.8(1)$ & $\mathrm{N} 13-\mathrm{C} 1-\mathrm{C} 6-\mathrm{N} 3$ & $-15.1(1)$ \\
$\mathrm{O} 1-\mathrm{N} 1-\mathrm{C} 2-\mathrm{C} 3$ & $116.9(1)$ & $\mathrm{C} 2-\mathrm{C} 1-\mathrm{C} 6-\mathrm{N} 3$ & $165.6(1)$ \\
$\mathrm{O} 2-\mathrm{N} 1-\mathrm{C} 2-\mathrm{C} 1$ & $126.2(1)$ & $\mathrm{O} 6-\mathrm{N} 3-\mathrm{C} 6-\mathrm{C} 5$ & $138.9(1)$ \\
$\mathrm{O} 1-\mathrm{N} 1-\mathrm{C} 2-\mathrm{C} 1$ & $-56.1(1)$ & $\mathrm{O} 5-\mathrm{N} 3-\mathrm{C} 6-\mathrm{C} 5$ & $-37.3(1)$ \\
$\mathrm{C} 5-\mathrm{C} 4-\mathrm{N} 2-\mathrm{O} 3$ & $-167.4(1)$ & $\mathrm{O} 6-\mathrm{N} 3-\mathrm{C} 6-\mathrm{C} 1$ & $-33.5(1)$ \\
$\mathrm{C} 3-\mathrm{C} 4-\mathrm{N} 2-\mathrm{O} 3$ & $12.9(1)$ & $\mathrm{O} 5-\mathrm{N} 3-\mathrm{C} 6-\mathrm{C} 1$ & $150.3(1)$
\end{tabular}

\subsection{Compound 5a}

Table S13. Details for X-ray data collection and structure refinement for compound 5a.

\begin{tabular}{ll}
\hline & 5a \\
\hline CCDC & 2025115 \\
Empirical formula & $\mathrm{C}_{8} \mathrm{H}_{8} \mathrm{FN}_{5} \mathrm{O}_{10} \mathrm{~S}$
\end{tabular}




\begin{tabular}{|c|c|}
\hline Formula mass & 385.25 \\
\hline $\mathrm{T}[\mathrm{K}]$ & $143(2)$ \\
\hline Crystal size $[\mathrm{mm}]$ & $0.30 \times 0.15 \times 0.10$ \\
\hline Crystal description & colorless needle \\
\hline Crystal system & monoclinic \\
\hline Space group & $P 21 / n$ \\
\hline $\mathrm{a}[\AA ̊ ́]$ & $7.3512(2)$ \\
\hline $\mathrm{b}[\AA ̊ 丿)$ & $9.4110(2)$ \\
\hline $\mathrm{c}[\AA \hat{A}]$ & $20.6030(5)$ \\
\hline$\alpha\left[^{\circ}\right]$ & 90.0 \\
\hline$\beta\left[^{\circ}\right]$ & $98.760(2)$ \\
\hline$\gamma\left[^{\circ}\right]$ & 90.0 \\
\hline $\mathrm{V}\left[\AA^{3}\right]$ & $1408.73(6)$ \\
\hline Z & 4 \\
\hline$\rho_{\text {calcd. }}\left[\mathrm{g} \mathrm{cm}^{-3}\right]$ & 1.816 \\
\hline$\mu\left[\mathrm{mm}^{-1}\right]$ & 0.313 \\
\hline$F(000)$ & 784 \\
\hline$\Theta$ range $\left[{ }^{\circ}\right]$ & $4.27-25.24$ \\
\hline \multirow[t]{3}{*}{ Index ranges } & $-10 \leq h \leq 10$ \\
\hline & $-13 \leq k \leq 13$ \\
\hline & $-29 \leq l \leq 29$ \\
\hline Reflns. collected & 27991 \\
\hline Reflns. obsd. & 3584 \\
\hline Reflns. unique & $\begin{array}{l}4285 \\
\left(R_{\text {int }}=0.0378\right)\end{array}$ \\
\hline$R_{1}, w R_{2}(2 \sigma$ data $)$ & $0.0332,0.0795$ \\
\hline$R_{1}, w R_{2}$ (all data) & $0.0432,0.0864$ \\
\hline GOOF on $F^{2}$ & 1.061 \\
\hline Peak/hole [e $\left.\AA^{-3}\right]$ & $0.374 /-0.405$ \\
\hline
\end{tabular}




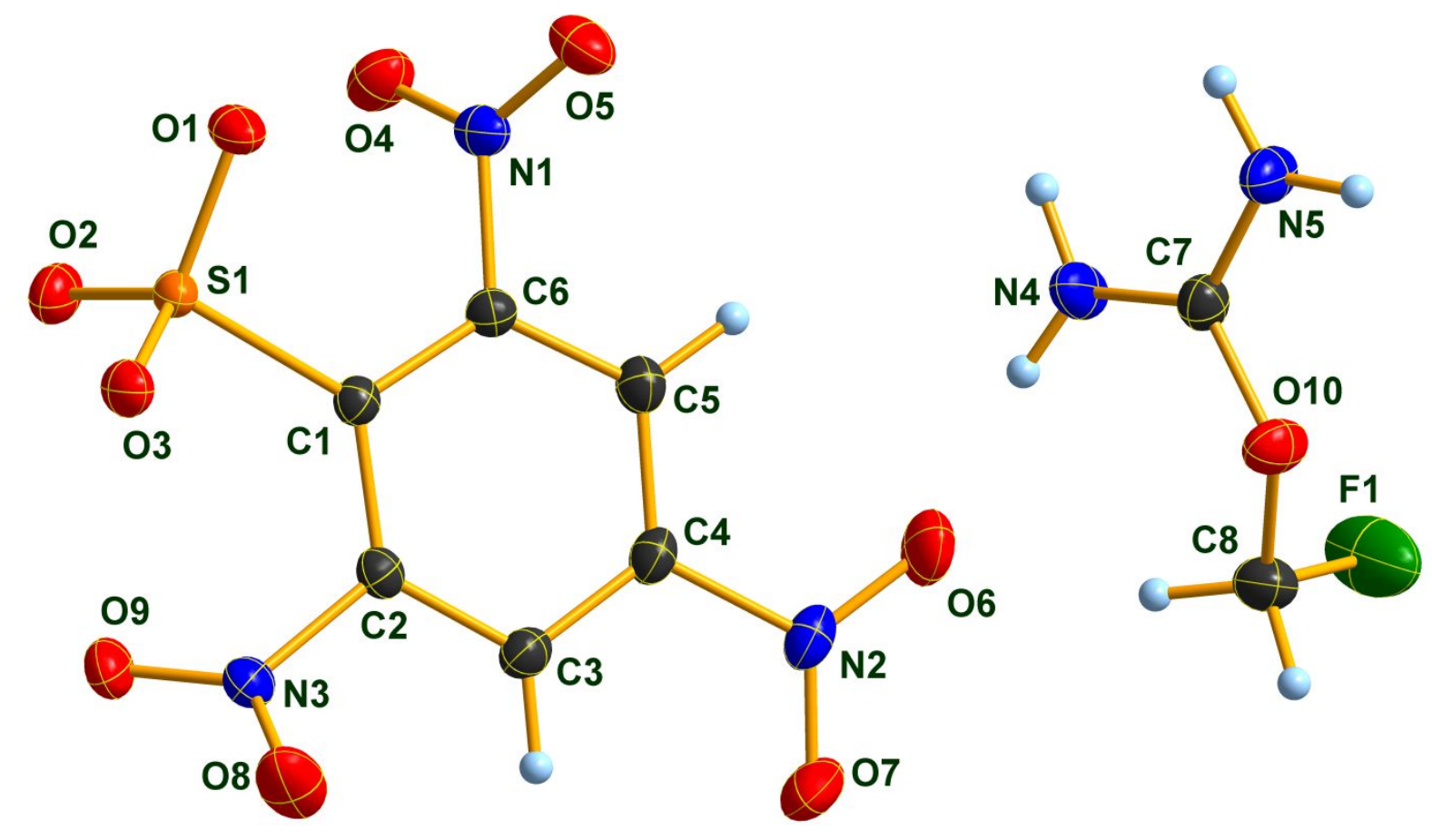

Figure S4. Molecular structure of compound 5a in the crystal. DIAMOND ${ }^{\mathrm{e})}$ representation; thermal ellipsoids are drawn at $50 \%$ probability level.

Table S14. Selected bond lengths $(\AA)$ of compound 5a.

$$
\begin{array}{ll|ll}
\mathrm{S} 1-\mathrm{O} 1 & 1.442(1) & \mathrm{N} 4-\mathrm{C} 7 & 1.307(2) \\
\mathrm{S} 1-\mathrm{O} 3 & 1.443(1) & \mathrm{C} 4-\mathrm{C} 5 & 1.379(2) \\
\mathrm{S} 1-\mathrm{O} 2 & 1.445(1) & \mathrm{C} 4-\mathrm{N} 2 & 1.477(2) \\
\mathrm{S} 1-\mathrm{C} 1 & 1.812(1) & \mathrm{O} 10-\mathrm{C} 7 & 1.330(2) \\
\mathrm{F} 1-\mathrm{C} 8 & 1.354(2) & \mathrm{O} 10-\mathrm{C} 8 & 1.411(2) \\
\mathrm{C} 1-\mathrm{C} 6 & 1.394(2) & \mathrm{O} 6-\mathrm{N} 2 & 1.219(2) \\
\mathrm{C} 1-\mathrm{C} 2 & 1.397(2) & \mathrm{C} 6-\mathrm{C} 5 & 1.385(2) \\
\mathrm{N} 1-\mathrm{O} 5 & 1.220(1) & \mathrm{O} 7-\mathrm{N} 2 & 1.219(2) \\
\mathrm{N} 1-\mathrm{O} 4 & 1.221(2) & \mathrm{C} 7-\mathrm{N} 5 & 1.303(2) \\
\mathrm{N} 1-\mathrm{C} 6 & 1.474(2) & \mathrm{N} 3-\mathrm{C} 2 & 1.477(2) \\
\mathrm{N} 3-\mathrm{O} 9 & 1.214(1) & \mathrm{C} 3-\mathrm{C} 4 & 1.379(2) \\
\mathrm{N} 3-\mathrm{O} 8 & 1.219(1) & \mathrm{C} 3-\mathrm{C} 2 & 1.384(2)
\end{array}
$$

Table S15. Selected bond angles $\left(^{\circ}\right)$ of compound 5a.

$$
\begin{array}{ll|ll}
\mathrm{O} 1-\mathrm{S} 1-\mathrm{O} 3 & 113.1(1) & \mathrm{C} 5-\mathrm{C} 6-\mathrm{C} 1 & 124.0(1) \\
\mathrm{O} 1-\mathrm{S} 1-\mathrm{O} 2 & 113.2(1) & \mathrm{C} 5-\mathrm{C} 6-\mathrm{N} 1 & 114.8(1) \\
\mathrm{O} 3-\mathrm{S} 1-\mathrm{O} 2 & 115.3(1) & \mathrm{C} 1-\mathrm{C} 6-\mathrm{N} 1 & 121.2(1) \\
\mathrm{O} 1-\mathrm{S} 1-\mathrm{C} 1 & 103.8(1) & \mathrm{N} 5-\mathrm{C} 7-\mathrm{N} 4 & 123.6(1)
\end{array}
$$




\begin{tabular}{ll|ll}
$\mathrm{O} 3-\mathrm{S} 1-\mathrm{C} 1$ & $104.8(1)$ & $\mathrm{N} 5-\mathrm{C} 7-\mathrm{O} 10$ & $113.5(1)$ \\
$\mathrm{O} 2-\mathrm{S} 1-\mathrm{C} 1$ & $105.3(1)$ & $\mathrm{N} 4-\mathrm{C} 7-\mathrm{O} 10$ & $122.9(1)$ \\
$\mathrm{C} 6-\mathrm{C} 1-\mathrm{C} 2$ & $115.2(1)$ & $\mathrm{C} 3-\mathrm{C} 2-\mathrm{C} 1$ & $123.5(1)$ \\
$\mathrm{C} 6-\mathrm{C} 1-\mathrm{S} 1$ & $121.1(1)$ & $\mathrm{C} 3-\mathrm{C} 2-\mathrm{N} 3$ & $115.4(1)$ \\
$\mathrm{C} 2-\mathrm{C} 1-\mathrm{S} 1$ & $123.5(1)$ & $\mathrm{C} 1-\mathrm{C} 2-\mathrm{N} 3$ & $121.1(1)$ \\
$\mathrm{O} 5-\mathrm{N} 1-\mathrm{O} 4$ & $125.6(1)$ & $\mathrm{O} 7-\mathrm{N} 2-\mathrm{O} 6$ & $124.9(1)$ \\
$\mathrm{O} 5-\mathrm{N} 1-\mathrm{C} 6$ & $117.1(1)$ & $\mathrm{O} 7-\mathrm{N} 2-\mathrm{C} 4$ & $117.7(1)$ \\
$\mathrm{O} 4-\mathrm{N} 1-\mathrm{C} 6$ & $117.2(1)$ & $\mathrm{O} 6-\mathrm{N} 2-\mathrm{C} 4$ & $117.4(1)$ \\
$\mathrm{O} 9-\mathrm{N} 3-\mathrm{O} 8$ & $125.4(1)$ & $\mathrm{F} 1-\mathrm{C} 8-\mathrm{O} 10$ & $108.1(1)$ \\
$\mathrm{O} 9-\mathrm{N} 3-\mathrm{C} 2$ & $117.7(1)$ & $\mathrm{C} 4-\mathrm{C} 5-\mathrm{C} 6$ & $117.1(1)$ \\
$\mathrm{O} 8-\mathrm{N} 3-\mathrm{C} 2$ & $116.8(1)$ & $\mathrm{C} 5-\mathrm{C} 4-\mathrm{N} 2$ & $118.5(1)$ \\
$\mathrm{C} 4-\mathrm{C} 3-\mathrm{C} 2$ & $117.6(1)$ & $\mathrm{C} 3-\mathrm{C} 4-\mathrm{N} 2$ & $118.9(1)$ \\
$\mathrm{C} 5-\mathrm{C} 4-\mathrm{C} 3$ & $122.7(1)$ & $\mathrm{C} 7-\mathrm{O} 10-\mathrm{C} 8$ & $120.5(1)$
\end{tabular}

Table S16. Selected torsion angles $\left(^{\circ}\right)$ of compound 5a.

\begin{tabular}{ll|ll}
$\mathrm{O} 1-\mathrm{S} 1-\mathrm{C} 1-\mathrm{C} 6$ & $-22.8(1)$ & $\mathrm{C} 4-\mathrm{C} 3-\mathrm{C} 2-\mathrm{N} 3$ & $-179.0(1)$ \\
$\mathrm{O} 3-\mathrm{S} 1-\mathrm{C} 1-\mathrm{C} 6$ & $-141.6(1)$ & $\mathrm{C} 6-\mathrm{C} 1-\mathrm{C} 2-\mathrm{C} 3$ & $0.0(2)$ \\
$\mathrm{O} 2-\mathrm{S} 1-\mathrm{C} 1-\mathrm{C} 6$ & $96.5(1)$ & $\mathrm{S} 1-\mathrm{C} 1-\mathrm{C} 2-\mathrm{C} 3$ & $-174.1(1)$ \\
$\mathrm{O} 1-\mathrm{S} 1-\mathrm{C} 1-\mathrm{C} 2$ & $150.9(1)$ & $\mathrm{C} 6-\mathrm{C} 1-\mathrm{C} 2-\mathrm{N} 3$ & $179.8(1)$ \\
$\mathrm{O} 3-\mathrm{S} 1-\mathrm{C} 1-\mathrm{C} 2$ & $32.1(1)$ & $\mathrm{S} 1-\mathrm{C} 1-\mathrm{C} 2-\mathrm{N} 3$ & $5.8(2)$ \\
$\mathrm{O} 2-\mathrm{S} 1-\mathrm{C} 1-\mathrm{C} 2$ & $-89.9(1)$ & $\mathrm{O} 9-\mathrm{N} 3-\mathrm{C} 2-\mathrm{C} 3$ & $-129.7(1)$ \\
$\mathrm{C} 2-\mathrm{C} 3-\mathrm{C} 4-\mathrm{C} 5$ & $-0.3(2)$ & $\mathrm{O} 8-\mathrm{N} 3-\mathrm{C} 2-\mathrm{C} 3$ & $48.3(2)$ \\
$\mathrm{C} 2-\mathrm{C} 3-\mathrm{C} 4-\mathrm{N} 2$ & $179.5(1)$ & $\mathrm{O} 9-\mathrm{N} 3-\mathrm{C} 2-\mathrm{C} 1$ & $50.5(2)$ \\
$\mathrm{C} 2-\mathrm{C} 1-\mathrm{C} 6-\mathrm{C} 5$ & $-1.5(2)$ & $\mathrm{O} 8-\mathrm{N} 3-\mathrm{C} 2-\mathrm{C} 1$ & $-131.6(1)$ \\
$\mathrm{S} 1-\mathrm{C} 1-\mathrm{C} 6-\mathrm{C} 5$ & $172.7(1)$ & $\mathrm{C} 5-\mathrm{C} 4-\mathrm{N} 2-\mathrm{O} 7$ & $-171.5(1)$ \\
$\mathrm{C} 2-\mathrm{C} 1-\mathrm{C} 6-\mathrm{N} 1$ & $177.9(1)$ & $\mathrm{C} 3-\mathrm{C} 4-\mathrm{N} 2-\mathrm{O} 7$ & $8.7(2)$ \\
$\mathrm{S} 1-\mathrm{C} 1-\mathrm{C} 6-\mathrm{N} 1$ & $-7.9(2)$ & $\mathrm{C} 5-\mathrm{C} 4-\mathrm{N} 2-\mathrm{O} 6$ & $9.3(2)$ \\
$\mathrm{O} 5-\mathrm{N} 1-\mathrm{C} 6-\mathrm{C} 5$ & $-61.5(2)$ & $\mathrm{C} 3-\mathrm{C} 4-\mathrm{N} 2-\mathrm{O} 6$ & $-170.5(1)$ \\
$\mathrm{O} 4-\mathrm{N} 1-\mathrm{C} 6-\mathrm{C} 5$ & $115.1(1)$ & $\mathrm{C} 7-\mathrm{O} 10-\mathrm{C} 8-\mathrm{F} 1$ & $75.9(2)$ \\
$\mathrm{O} 5-\mathrm{N} 1-\mathrm{C} 6-\mathrm{C} 1$ & $119.1(1)$ & $\mathrm{C} 3-\mathrm{C} 4-\mathrm{C} 5-\mathrm{C} 6$ & $-1.0(2)$ \\
$\mathrm{O} 4-\mathrm{N} 1-\mathrm{C} 6-\mathrm{C} 1$ & $-64.3(2)$ & $\mathrm{N} 2-\mathrm{C} 4-\mathrm{C} 5-\mathrm{C} 6$ & $179.2(1)$ \\
$\mathrm{C} 8-\mathrm{O} 10-\mathrm{C} 7-\mathrm{N} 5$ & $-156.7(1)$ & $\mathrm{C} 1-\mathrm{C} 6-\mathrm{C} 5-\mathrm{C} 4$ & $2.0(2)$ \\
$\mathrm{C} 8-\mathrm{O} 10-\mathrm{C} 7-\mathrm{N} 4$ & $23.8(2)$ & $\mathrm{N} 1-\mathrm{C} 6-\mathrm{C} 5-\mathrm{C} 4$ & $-177.4(1)$ \\
$\mathrm{C} 4-\mathrm{C} 3-\mathrm{C} 2-\mathrm{C} 1$ & $0.9(2)$ & & \\
& & &
\end{tabular}

\subsection{Compound 6a}

Table S17. Details for X-ray data collection and structure refinement for compound $\mathbf{6 a}$. 


\begin{tabular}{|c|c|}
\hline & $\mathbf{6 a}$ \\
\hline $\mathrm{CCDC}$ & 2025116 \\
\hline Empirical formula & $\mathrm{C}_{6} \mathrm{H}_{6} \mathrm{~N}_{4} \mathrm{O}_{9} \mathrm{~S}$ \\
\hline Formula mass & 310.21 \\
\hline $\mathrm{T}[\mathrm{K}]$ & $143(2)$ \\
\hline Crystal size [mm] & $0.15 \times 0.15 \times 0.02$ \\
\hline Crystal description & colorless platelet \\
\hline Crystal system & orthorhombic \\
\hline Space group & Pbca \\
\hline $\mathrm{a}[\AA ̊ 丿]$ & $8.4167(3)$ \\
\hline $\mathrm{b}[\AA ̊ d]$ & $9.3286(4)$ \\
\hline $\mathrm{c}[\AA \hat{x}]$ & $28.5429(13)$ \\
\hline$\alpha\left[^{\circ}\right]$ & 90.0 \\
\hline$\beta\left[^{\circ}\right]$ & 90.0 \\
\hline$\gamma\left[^{\circ}\right]$ & 90.0 \\
\hline $\mathrm{V}\left[\AA^{3}\right]$ & $2241.08(16)$ \\
\hline $\mathrm{Z}$ & 8 \\
\hline$\rho_{\text {calcd. }}\left[\mathrm{g} \mathrm{cm}^{-3}\right]$ & 1.839 \\
\hline$\mu\left[\mathrm{mm}^{-1}\right]$ & 0.348 \\
\hline$F(000)$ & 1264 \\
\hline$\Theta$ range $\left[{ }^{\circ}\right]$ & $4.28-25.24$ \\
\hline \multirow[t]{3}{*}{ Index ranges } & $-10 \leq h \leq 6$ \\
\hline & $-11 \leq k \leq 11$ \\
\hline & $-35 \leq l \leq 35$ \\
\hline Reflns. collected & 16780 \\
\hline Reflns. obsd. & 1836 \\
\hline Reflns. unique & $\begin{array}{l}2287 \\
\left(\mathrm{R}_{\mathrm{int}}=0.0551\right)\end{array}$ \\
\hline$R_{1}, w R_{2}(2 \sigma$ data $)$ & $0.0355,0.0791$ \\
\hline$R_{1}, w R_{2}$ (all data) & $0.0488,0.0864$ \\
\hline GOOF on $F^{2}$ & 1.040 \\
\hline Peak/hole [e $\left.\AA^{-3}\right]$ & $0.356 /-0.326$ \\
\hline
\end{tabular}




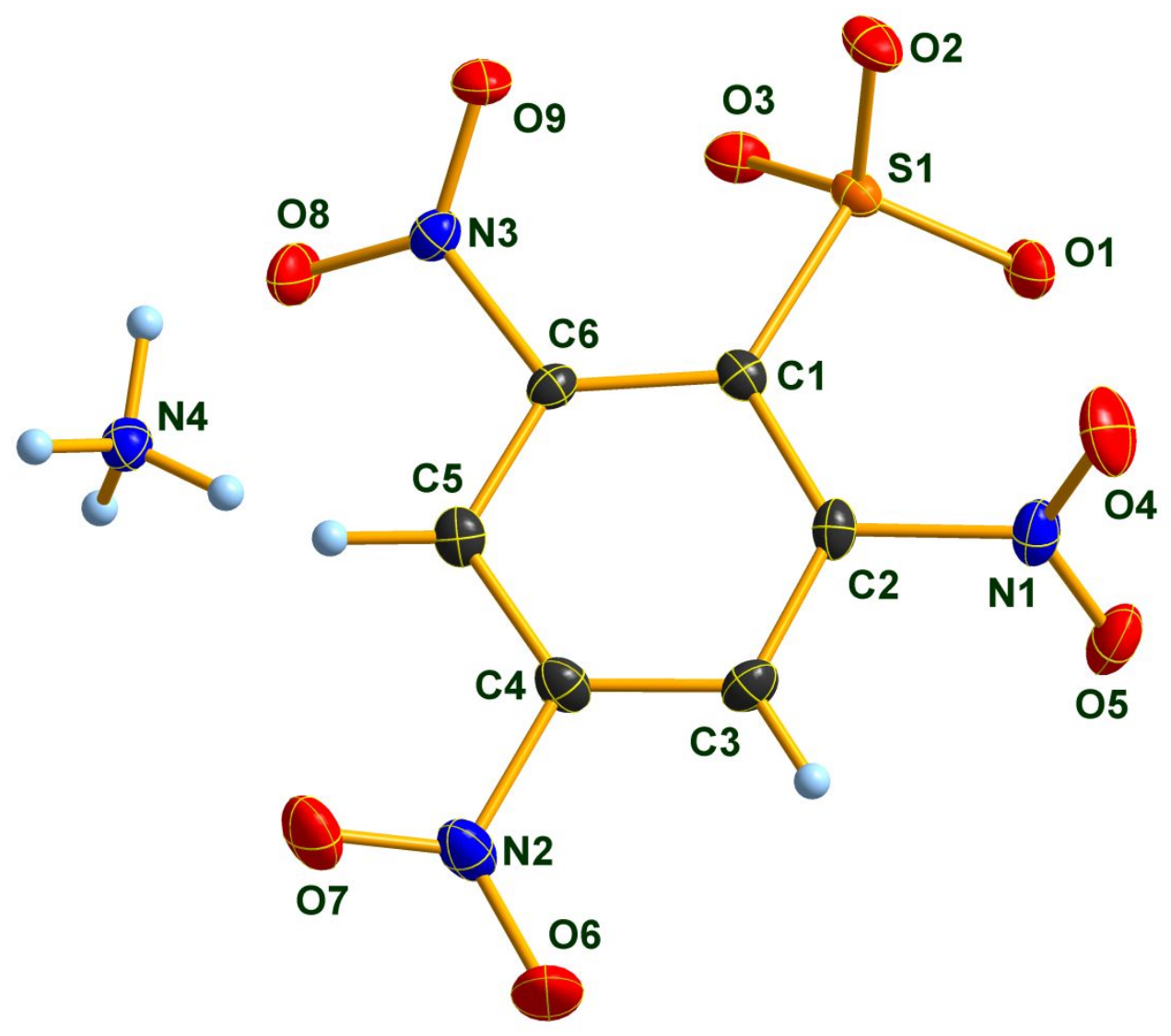

Figure S5. Molecular structure of compound 6a in the crystal. DIAMOND ${ }^{\mathrm{e})}$ representation; thermal ellipsoids are drawn at $50 \%$ probability level.

Table S18. Selected bond lengths $(\AA)$ of compound $\mathbf{6 a}$.

\begin{tabular}{ll|ll}
$\mathrm{S} 1-\mathrm{O} 1$ & $1.443(2)$ & $\mathrm{N} 3-\mathrm{O} 9$ & $1.220(2)$ \\
$\mathrm{S} 1-\mathrm{O} 2$ & $1.444(2)$ & $\mathrm{N} 3-\mathrm{O} 8$ & $1.223(2)$ \\
$\mathrm{S} 1-\mathrm{O} 3$ & $1.454(2)$ & $\mathrm{N} 3-\mathrm{C} 6$ & $1.476(3)$ \\
$\mathrm{S} 1-\mathrm{C} 1$ & $1.813(2)$ & $\mathrm{C} 3-\mathrm{C} 4$ & $1.378(3)$ \\
$\mathrm{N} 1-\mathrm{O} 5$ & $1.216(2)$ & $\mathrm{C} 6-\mathrm{C} 5$ & $1.382(3)$ \\
$\mathrm{N} 1-\mathrm{O} 4$ & $1.226(2)$ & $\mathrm{C} 5-\mathrm{C} 4$ & $1.380(3)$ \\
$\mathrm{N} 1-\mathrm{C} 2$ & $1.486(3)$ & $\mathrm{N} 2-\mathrm{O} 6$ & $1.222(2)$ \\
$\mathrm{C} 1-\mathrm{C} 2$ & $1.400(3)$ & $\mathrm{N} 2-\mathrm{O} 7$ & $1.222(2)$ \\
$\mathrm{C} 1-\mathrm{C} 6$ & $1.401(3)$ & $\mathrm{N} 2-\mathrm{C} 4$ & $1.476(3)$ \\
$\mathrm{C} 2-\mathrm{C} 3$ & $1.380(3)$ & &
\end{tabular}

Table S19. Selected bond angles $\left(^{\circ}\right)$ of compound $6 \mathbf{a}$.

$$
\mathrm{O} 1-\mathrm{S} 1-\mathrm{O} 2 \quad 113.5(1) \mid \mathrm{O} 8-\mathrm{N} 3-\mathrm{C} 6 \quad 116.8(2)
$$




$$
\begin{array}{ll|ll}
\mathrm{O} 1-\mathrm{S} 1-\mathrm{O} 3 & 113.2(1) & \mathrm{C} 4-\mathrm{C} 3-\mathrm{C} 2 & 117.3(2) \\
\mathrm{O} 2-\mathrm{S} 1-\mathrm{O} 3 & 113.6(1) & \mathrm{C} 5-\mathrm{C} 6-\mathrm{C} 1 & 123.8(2) \\
\mathrm{O} 1-\mathrm{S} 1-\mathrm{C} 1 & 104.7(1) & \mathrm{C} 5-\mathrm{C} 6-\mathrm{N} 3 & 114.8(2) \\
\mathrm{O} 2-\mathrm{S} 1-\mathrm{C} 1 & 107.7(1) & \mathrm{C} 1-\mathrm{C} 6-\mathrm{N} 3 & 121.3(2) \\
\mathrm{O} 3-\mathrm{S} 1-\mathrm{C} 1 & 103.0(1) & \mathrm{C} 4-\mathrm{C} 5-\mathrm{C} 6 & 117.0(2) \\
\mathrm{O} 5-\mathrm{N} 1-\mathrm{O} 4 & 126.1(2) & \mathrm{C} 3-\mathrm{C} 4-\mathrm{C} 5 & 123.1(2) \\
\mathrm{O} 5-\mathrm{N} 1-\mathrm{C} 2 & 117.6(2) & \mathrm{C} 3-\mathrm{C} 4-\mathrm{N} 2 & 119.0(2) \\
\mathrm{O} 4-\mathrm{N} 1-\mathrm{C} 2 & 116.2(2) & \mathrm{C} 5-\mathrm{C} 4-\mathrm{N} 2 & 117.9(2) \\
\mathrm{C} 2-\mathrm{C} 1-\mathrm{C} 6 & 115.0(2) & \mathrm{O} 6-\mathrm{N} 2-\mathrm{C} 4 & 117.2(2) \\
\mathrm{C} 2-\mathrm{C} 1-\mathrm{S} 1 & 121.2(2) & \mathrm{O} 7-\mathrm{N} 2-\mathrm{C} 4 & 117.5(2) \\
\mathrm{C} 6-\mathrm{C} 1-\mathrm{S} 1 & 122.6(2) & \mathrm{O} 9-\mathrm{N} 3-\mathrm{O} 8 & 124.8(2) \\
\mathrm{C} 3-\mathrm{C} 2-\mathrm{C} 1 & 123.7(2) & \mathrm{O} 9-\mathrm{N} 3-\mathrm{C} 6 & 118.3(2) \\
\mathrm{C} 3-\mathrm{C} 2-\mathrm{N} 1 & 115.3(2) & \mathrm{O} 6-\mathrm{N} 2-\mathrm{O} 7 & 125.2(2) \\
\mathrm{C} 1-\mathrm{C} 2-\mathrm{N} 1 & 121.0(2) & &
\end{array}
$$

Table S20. Selected torsion angles $\left(^{\circ}\right)$ of compound $\mathbf{6 a}$.

\begin{tabular}{ll|ll}
$\mathrm{O} 1-\mathrm{S} 1-\mathrm{C} 1-\mathrm{C} 2$ & $14.5(2)$ & $\mathrm{S} 1-\mathrm{C} 1-\mathrm{C} 6-\mathrm{C} 5$ & $168.8(2)$ \\
$\mathrm{O} 2-\mathrm{S} 1-\mathrm{C} 1-\mathrm{C} 2$ & $-106.6(2)$ & $\mathrm{C} 2-\mathrm{C} 1-\mathrm{C} 6-\mathrm{N} 3$ & $-179.7(2)$ \\
$\mathrm{O} 3-\mathrm{S} 1-\mathrm{C} 1-\mathrm{C} 2$ & $133.1(2)$ & $\mathrm{S} 1-\mathrm{C} 1-\mathrm{C} 6-\mathrm{N} 3$ & $-10.1(3)$ \\
$\mathrm{O} 1-\mathrm{S} 1-\mathrm{C} 1-\mathrm{C} 6$ & $-154.5(2)$ & $\mathrm{O} 9-\mathrm{N} 3-\mathrm{C} 6-\mathrm{C} 5$ & $140.0(2)$ \\
$\mathrm{O} 2-\mathrm{S} 1-\mathrm{C} 1-\mathrm{C} 6$ & $84.5(2)$ & $\mathrm{O} 8-\mathrm{N} 3-\mathrm{C} 6-\mathrm{C} 5$ & $-37.5(2)$ \\
$\mathrm{O} 3-\mathrm{S} 1-\mathrm{C} 1-\mathrm{C} 6$ & $-35.8(2)$ & $\mathrm{O} 9-\mathrm{N} 3-\mathrm{C} 6-\mathrm{C} 1$ & $-41.1(3)$ \\
$\mathrm{C} 6-\mathrm{C} 1-\mathrm{C} 2-\mathrm{C} 3$ & $3.3(3)$ & $\mathrm{O} 8-\mathrm{N} 3-\mathrm{C} 6-\mathrm{C} 1$ & $141.5(2)$ \\
$\mathrm{S} 1-\mathrm{C} 1-\mathrm{C} 2-\mathrm{C} 3$ & $-166.4(2)$ & $\mathrm{C} 1-\mathrm{C} 6-\mathrm{C} 5-\mathrm{C} 4$ & $-1.8(3)$ \\
$\mathrm{C} 6-\mathrm{C} 1-\mathrm{C} 2-\mathrm{N} 1$ & $-176.1(2)$ & $\mathrm{N} 3-\mathrm{C} 6-\mathrm{C} 5-\mathrm{C} 4$ & $177.2(2)$ \\
$\mathrm{S} 1-\mathrm{C} 1-\mathrm{C} 2-\mathrm{N} 1$ & $14.2(3)$ & $\mathrm{C} 2-\mathrm{C} 3-\mathrm{C} 4-\mathrm{C} 5$ & $0.2(3)$ \\
$\mathrm{O} 5-\mathrm{N} 1-\mathrm{C} 2-\mathrm{C} 3$ & $64.0(2)$ & $\mathrm{C} 2-\mathrm{C} 3-\mathrm{C} 4-\mathrm{N} 2$ & $179.6(2)$ \\
$\mathrm{O} 4-\mathrm{N} 1-\mathrm{C} 2-\mathrm{C} 3$ & $-113.2(2)$ & $\mathrm{C} 6-\mathrm{C} 5-\mathrm{C} 4-\mathrm{C} 3$ & $2.1(3)$ \\
$\mathrm{O} 5-\mathrm{N} 1-\mathrm{C} 2-\mathrm{C} 1$ & $-116.6(2)$ & $\mathrm{C} 6-\mathrm{C} 5-\mathrm{C} 4-\mathrm{N} 2$ & $-177.3(2)$ \\
$\mathrm{O} 4-\mathrm{N} 1-\mathrm{C} 2-\mathrm{C} 1$ & $66.2(3)$ & $\mathrm{O} 6-\mathrm{N} 2-\mathrm{C} 4-\mathrm{C} 3$ & $-23.5(3)$ \\
$\mathrm{C} 1-\mathrm{C} 2-\mathrm{C} 3-\mathrm{C} 4$ & $-3.0(3)$ & $\mathrm{O} 7-\mathrm{N} 2-\mathrm{C} 4-\mathrm{C} 3$ & $158.1(2)$ \\
$\mathrm{N} 1-\mathrm{C} 2-\mathrm{C} 3-\mathrm{C} 4$ & $176.4(2)$ & $\mathrm{O} 6-\mathrm{N} 2-\mathrm{C} 4-\mathrm{C} 5$ & $155.9(2)$ \\
$\mathrm{C} 2-\mathrm{C} 1-\mathrm{C} 6-\mathrm{C} 5$ & $-0.8(3)$ & $\mathrm{O} 7-\mathrm{N} 2-\mathrm{C} 4-\mathrm{C} 5$ & $-22.5(3)$
\end{tabular}

2.6 Compound $\mathbf{6 a} * \mathrm{H}_{2} \mathrm{O}$

Table S21. Details for X-ray data collection and structure refinement for compound $6 \mathbf{a} \cdot \mathbf{H}_{2} \mathbf{O}$. 


\begin{tabular}{|c|c|}
\hline & $6 \mathrm{a} \cdot \mathrm{H}_{2} \mathrm{O}$ \\
\hline CCDC & 2025109 \\
\hline Empirical formula & $\mathrm{C}_{6} \mathrm{H}_{8} \mathrm{~N}_{4} \mathrm{O}_{10} \mathrm{~S}$ \\
\hline Formula mass & 328.22 \\
\hline $\mathrm{T}[\mathrm{K}]$ & $143(2)$ \\
\hline Crystal size [mm] & $0.217 \times 0.176 \times 0.052$ \\
\hline Crystal description & colorless block \\
\hline Crystal system & triclinic \\
\hline Space group & $P-1$ \\
\hline 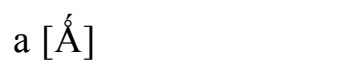 & $7.8975(10)$ \\
\hline b [Á] & $8.2772(8)$ \\
\hline 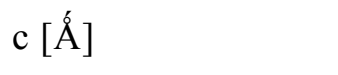 & $10.1691(9)$ \\
\hline$\alpha\left[^{\circ}\right]$ & $73.685(8)$ \\
\hline$\beta\left[^{\circ}\right]$ & $80.191(9)$ \\
\hline$\gamma\left[^{\circ}\right]$ & $66.637(10)$ \\
\hline $\mathrm{V}\left[\AA^{3}\right]$ & $584.36(12)$ \\
\hline $\mathrm{Z}$ & 2 \\
\hline$\rho_{\text {calcd. }}\left[\mathrm{g} \mathrm{cm}^{-3}\right]$ & 1.865 \\
\hline$\mu\left[\mathrm{mm}^{-1}\right]$ & 0.345 \\
\hline$F(000)$ & 336 \\
\hline$\Theta$ range $\left[{ }^{\circ}\right]$ & $4.19-25.24$ \\
\hline \multirow[t]{3}{*}{ Index ranges } & $-10 \leq h \leq 11$ \\
\hline & $-11 \leq k \leq 11$ \\
\hline & $-14 \leq l \leq 14$ \\
\hline Reflns. collected & 6040 \\
\hline Reflns. obsd. & 2737 \\
\hline Reflns. unique & $\begin{array}{l}3547 \\
\left(\mathrm{R}_{\mathrm{int}}=0.0370\right)\end{array}$ \\
\hline$R_{1}, w R_{2}(2 \sigma$ data $)$ & $0.0459,0.0971$ \\
\hline$R_{1}, w R_{2}$ (all data) & $0.0634,0.1132$ \\
\hline GOOF on $F^{2}$ & 1.049 \\
\hline Peak/hole [e $\AA^{-3}$ ] & $0.479 /-0.602$ \\
\hline
\end{tabular}




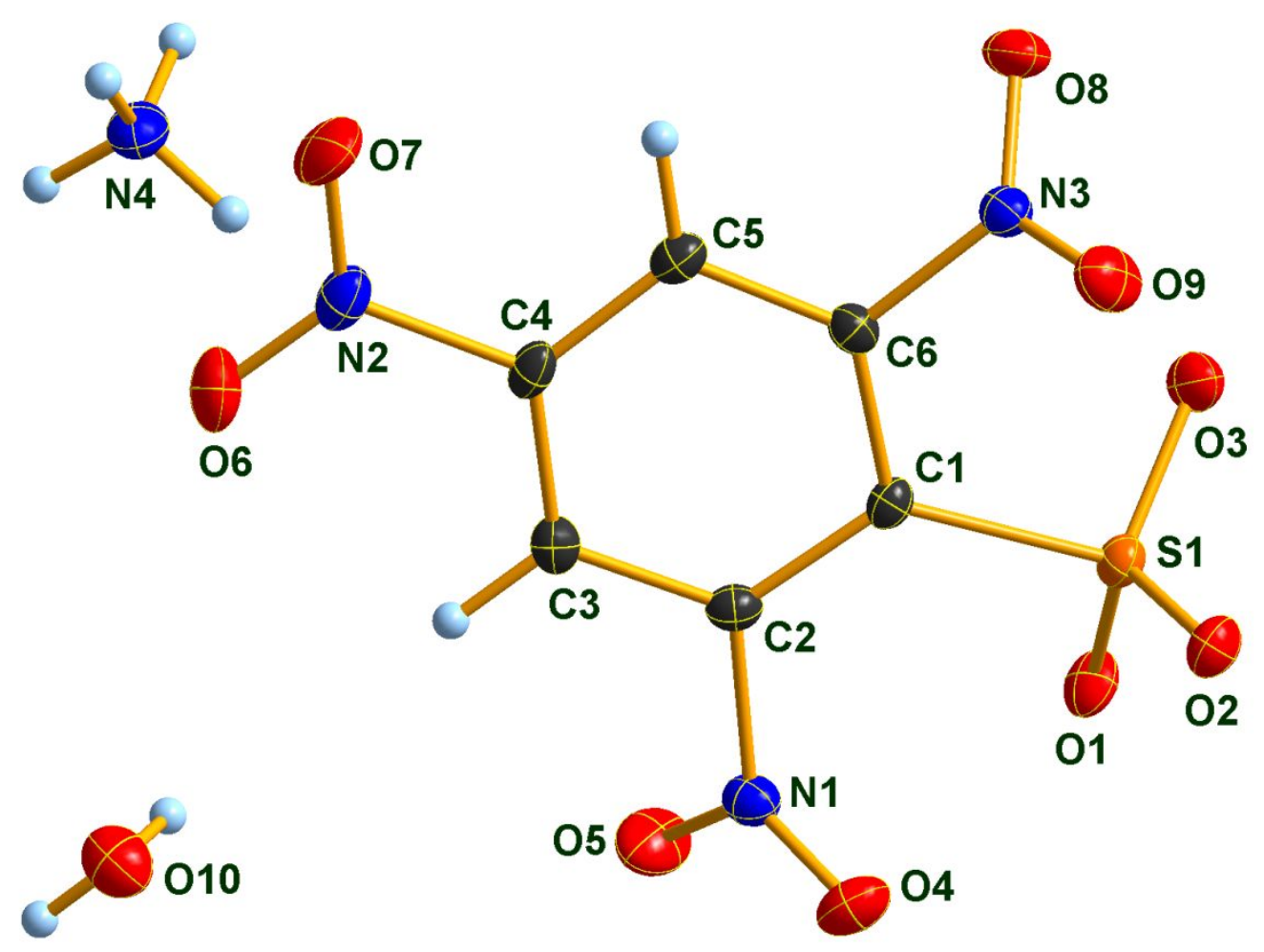

Figure S6. Molecular structure of compound $\mathbf{6 a} \cdot \mathbf{H}_{2} \mathbf{O}$ in the crystal. DIAMOND ${ }^{\mathrm{e}}$ representation; thermal ellipsoids are drawn at $50 \%$ probability level.

Table S22. Selected bond lengths $(\AA)$ of compound $\mathbf{6 a} \cdot \mathbf{H}_{2} \mathbf{O}$.

$$
\begin{array}{ll|ll}
\mathrm{S} 1-\mathrm{O} 1 & 1.439(1) & \mathrm{C} 4-\mathrm{C} 3 & 1.377(2) \\
\mathrm{S} 1-\mathrm{O} 2 & 1.443(1) & \mathrm{O} 9-\mathrm{N} 3 & 1.229(2) \\
\mathrm{S} 1-\mathrm{O} 3 & 1.448(1) & \mathrm{N} 3-\mathrm{O} 8 & 1.216(2) \\
\mathrm{S} 1-\mathrm{C} 1 & 1.815(2) & \mathrm{N} 3-\mathrm{C} 6 & 1.471(2) \\
\mathrm{C} 1-\mathrm{C} 2 & 1.389(2) & \mathrm{C} 6-\mathrm{C} 5 & 1.380(2) \\
\mathrm{C} 1-\mathrm{C} 6 & 1.400(2) & \mathrm{N} 2-\mathrm{O} 6 & 1.216(2) \\
\mathrm{N} 1-\mathrm{O} 4 & 1.217(2) & \mathrm{N} 2-\mathrm{O} 7 & 1.220(2) \\
\mathrm{N} 1-\mathrm{O} 5 & 1.225(2) & \mathrm{N} 2-\mathrm{C} 4 & 1.476(2) \\
\mathrm{N} 1-\mathrm{C} 2 & 1.482(2) & \mathrm{C} 4-\mathrm{C} 5 & 1.375(2) \\
\mathrm{C} 2-\mathrm{C} 3 & 1.382(2) & &
\end{array}
$$

Table S23. Selected bond angles $\left(^{\circ}\right)$ of compound $6 \mathbf{6} \cdot \mathbf{H}_{2} \mathbf{O}$.

$$
\begin{array}{ll|ll}
\mathrm{O} 1-\mathrm{S} 1-\mathrm{O} 2 & 114.1(1) & \mathrm{C} 3-\mathrm{C} 4-\mathrm{N} 2 & 119.2(2) \\
\mathrm{O} 1-\mathrm{S} 1-\mathrm{O} 3 & 113.9(1) & \mathrm{O} 8-\mathrm{N} 3-\mathrm{O} 9 & 124.9(2) \\
\mathrm{O} 2-\mathrm{S} 1-\mathrm{O} 3 & 114.1(1) & \mathrm{O} 8-\mathrm{N} 3-\mathrm{C} 6 & 118.4(2) \\
\mathrm{O} 1-\mathrm{S} 1-\mathrm{C} 1 & 104.1(1) & \mathrm{O} 9-\mathrm{N} 3-\mathrm{C} 6 & 116.6(1) \\
\mathrm{O} 2-\mathrm{S} 1-\mathrm{C} 1 & 106.6(1) & \mathrm{C} 4-\mathrm{C} 3-\mathrm{C} 2 & 117.7(2)
\end{array}
$$




$$
\begin{array}{ll|ll}
\mathrm{O} 3-\mathrm{S} 1-\mathrm{C} 1 & 102.5(1) & \mathrm{C} 5-\mathrm{C} 6-\mathrm{C} 1 & 124.1(2) \\
\mathrm{C} 2-\mathrm{C} 1-\mathrm{C} 6 & 114.9(2) & \mathrm{C} 5-\mathrm{C} 6-\mathrm{N} 3 & 115.4(2) \\
\mathrm{C} 2-\mathrm{C} 1-\mathrm{S} 1 & 123.8(1) & \mathrm{C} 1-\mathrm{C} 6-\mathrm{N} 3 & 120.4(2) \\
\mathrm{C} 6-\mathrm{C} 1-\mathrm{S} 1 & 120.3(1) & \mathrm{C} 4-\mathrm{C} 5-\mathrm{C} 6 & 117.1(2) \\
\mathrm{O} 4-\mathrm{N} 1-\mathrm{O} 5 & 125.3(2) & \mathrm{O} 6-\mathrm{N} 2-\mathrm{O} 7 & 125.3(2) \\
\mathrm{O} 4-\mathrm{N} 1-\mathrm{C} 2 & 118.2(2) & \mathrm{O} 6-\mathrm{N} 2-\mathrm{C} 4 & 117.6(2) \\
\mathrm{O} 5-\mathrm{N} 1-\mathrm{C} 2 & 116.5(2) & \mathrm{O} 7-\mathrm{N} 2-\mathrm{C} 4 & 117.1(2) \\
\mathrm{C} 3-\mathrm{C} 2-\mathrm{C} 1 & 123.6(2) & \mathrm{C} 5-\mathrm{C} 4-\mathrm{C} 3 & 122.6(2) \\
\mathrm{C} 3-\mathrm{C} 2-\mathrm{N} 1 & 115.5(2) & \mathrm{C} 5-\mathrm{C} 4-\mathrm{N} 2 & 118.2(2) \\
\mathrm{C} 1-\mathrm{C} 2-\mathrm{N} 1 & 120.9(2) & &
\end{array}
$$

Table S24. Selected torsion angles $\left(^{\circ}\right)$ of compound $6 \mathbf{6 a} \cdot \mathbf{H}_{2} \mathbf{O}$.

\begin{tabular}{ll|ll}
$\mathrm{O} 1-\mathrm{S} 1-\mathrm{C} 1-\mathrm{C} 2$ & $20.0(2)$ & $\mathrm{O} 7-\mathrm{N} 2-\mathrm{C} 4-\mathrm{C} 3$ & $172.3(2)$ \\
$\mathrm{O} 2-\mathrm{S} 1-\mathrm{C} 1-\mathrm{C} 2$ & $-101.0(2)$ & $\mathrm{C} 5-\mathrm{C} 4-\mathrm{C} 3-\mathrm{C} 2$ & $-0.4(3)$ \\
$\mathrm{O} 3-\mathrm{S} 1-\mathrm{C} 1-\mathrm{C} 2$ & $138.9(2)$ & $\mathrm{N} 2-\mathrm{C} 4-\mathrm{C} 3-\mathrm{C} 2$ & $-180.0(2)$ \\
$\mathrm{O} 1-\mathrm{S} 1-\mathrm{C} 1-\mathrm{C} 6$ & $-147.7(1)$ & $\mathrm{C} 1-\mathrm{C} 2-\mathrm{C} 3-\mathrm{C} 4$ & $-1.0(3)$ \\
$\mathrm{O} 2-\mathrm{S} 1-\mathrm{C} 1-\mathrm{C} 6$ & $91.3(2)$ & $\mathrm{N} 1-\mathrm{C} 2-\mathrm{C} 3-\mathrm{C} 4$ & $177.3(2)$ \\
$\mathrm{O} 3-\mathrm{S} 1-\mathrm{C} 1-\mathrm{C} 6$ & $-28.8(2)$ & $\mathrm{C} 2-\mathrm{C} 1-\mathrm{C} 6-\mathrm{C} 5$ & $-3.1(3)$ \\
$\mathrm{C} 6-\mathrm{C} 1-\mathrm{C} 2-\mathrm{C} 3$ & $2.6(3)$ & $\mathrm{S} 1-\mathrm{C} 1-\mathrm{C} 6-\mathrm{C} 5$ & $165.7(2)$ \\
$\mathrm{S} 1-\mathrm{C} 1-\mathrm{C} 2-\mathrm{C} 3$ & $-165.7(2)$ & $\mathrm{C} 2-\mathrm{C} 1-\mathrm{C} 6-\mathrm{N} 3$ & $174.2(1)$ \\
$\mathrm{C} 6-\mathrm{C} 1-\mathrm{C} 2-\mathrm{N} 1$ & $-175.6(2)$ & $\mathrm{S} 1-\mathrm{C} 1-\mathrm{C} 6-\mathrm{N} 3$ & $-17.1(2)$ \\
$\mathrm{S} 1-\mathrm{C} 1-\mathrm{C} 2-\mathrm{N} 1$ & $16.2(2)$ & $\mathrm{O} 8-\mathrm{N} 3-\mathrm{C} 6-\mathrm{C} 5$ & $-56.1(2)$ \\
$\mathrm{O} 4-\mathrm{N} 1-\mathrm{C} 2-\mathrm{C} 3$ & $-129.7(2)$ & $\mathrm{O} 9-\mathrm{N} 3-\mathrm{C} 6-\mathrm{C} 5$ & $120.4(2)$ \\
$\mathrm{O} 5-\mathrm{N} 1-\mathrm{C} 2-\mathrm{C} 3$ & $46.7(2)$ & $\mathrm{O} 8-\mathrm{N} 3-\mathrm{C} 6-\mathrm{C} 1$ & $126.5(2)$ \\
$\mathrm{O} 4-\mathrm{N} 1-\mathrm{C} 2-\mathrm{C} 1$ & $48.7(2)$ & $\mathrm{O} 9-\mathrm{N} 3-\mathrm{C} 6-\mathrm{C} 1$ & $-57.0(2)$ \\
$\mathrm{O} 5-\mathrm{N} 1-\mathrm{C} 2-\mathrm{C} 1$ & $-135.0(2)$ & $\mathrm{C} 3-\mathrm{C} 4-\mathrm{C} 5-\mathrm{C} 6$ & $0.0(3)$ \\
$\mathrm{O} 6-\mathrm{N} 2-\mathrm{C} 4-\mathrm{C} 5$ & $172.7(2)$ & $\mathrm{N} 2-\mathrm{C} 4-\mathrm{C} 5-\mathrm{C} 6$ & $179.6(2)$ \\
$\mathrm{O} 7-\mathrm{N} 2-\mathrm{C} 4-\mathrm{C} 5$ & $-7.3(3)$ & $\mathrm{C} 1-\mathrm{C} 6-\mathrm{C} 5-\mathrm{C} 4$ & $1.8(3)$ \\
$\mathrm{O} 6-\mathrm{N} 2-\mathrm{C} 4-\mathrm{C} 3$ & $-7.7(3)$ & $\mathrm{N} 3-\mathrm{C} 6-\mathrm{C} 5-\mathrm{C} 4$ & $-175.5(2)$
\end{tabular}

\subsection{Compound 5b}

Table S25. Details for X-ray data collection and structure refinement for compound $\mathbf{5 b}$. 


\begin{tabular}{ll}
\hline & $\mathbf{5 b}$ \\
\hline CCDC & 2025112 \\
Empirical formula & $\mathrm{C}_{11} \mathrm{H}_{13} \mathrm{FN}_{4} \mathrm{O}_{10} \mathrm{~S}$ \\
Formula mass & 412.31 \\
$\mathrm{~T}[\mathrm{~K}]$ & $143(2)$ \\
Crystal size [mm] & $0.25 \times 0.15 \times 0.07$ \\
Crystal description & colorless block \\
Crystal system & monoclinic \\
Space group & $P 21 / n$ \\
$\mathrm{a}[\AA \AA$ & \\
$\mathrm{b}[\AA ̊$ &
\end{tabular}




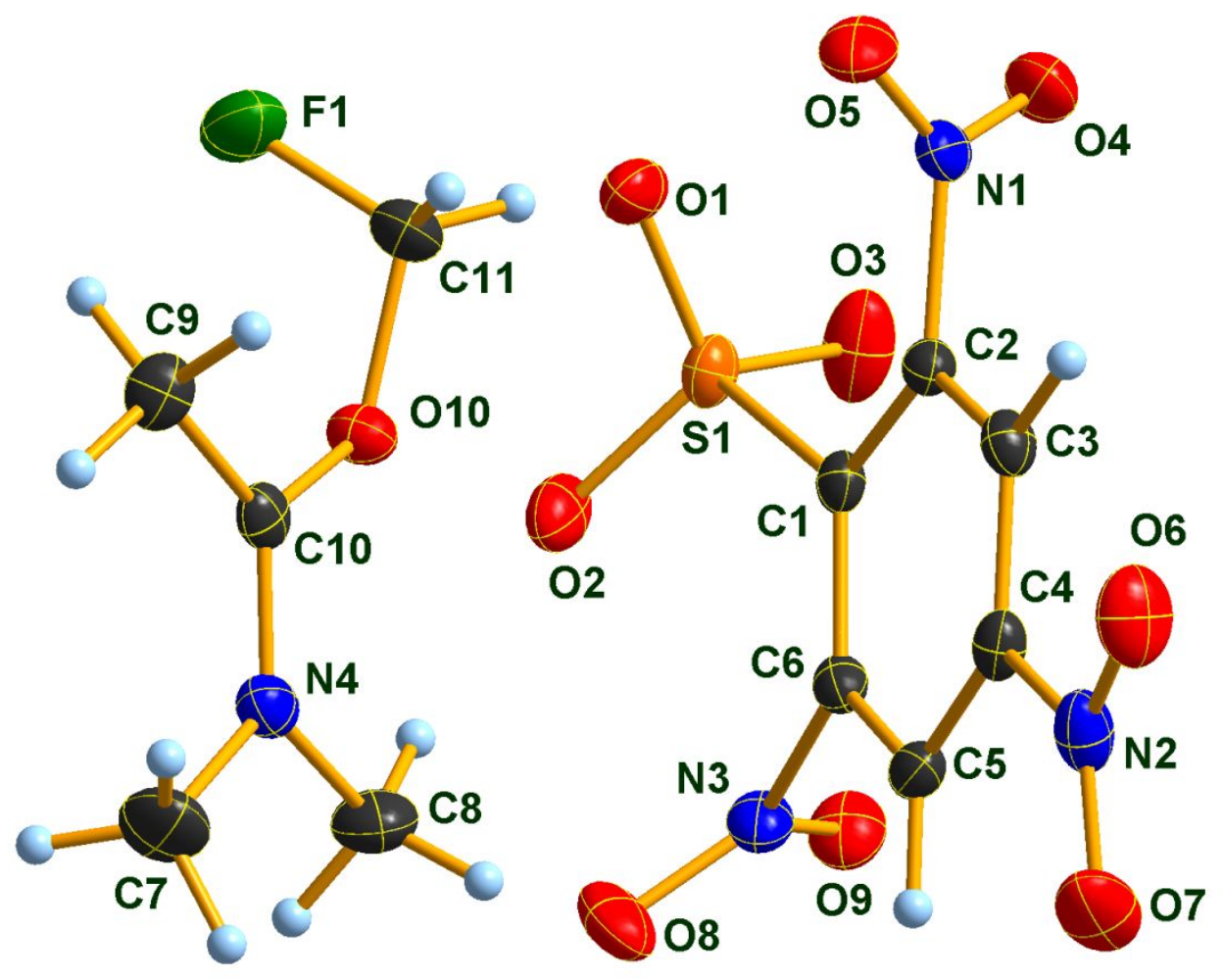

Figure S7. Molecular structure of compound $\mathbf{5 b}$ in the crystal. DIAMOND ${ }^{\mathrm{e})}$ representation; thermal ellipsoids are drawn at $50 \%$ probability level.

Table S26. Selected bond lengths ( $\AA$ ) of compound 5b.

$$
\begin{array}{ll|ll}
\mathrm{S} 1-\mathrm{O} 3 & 1.436(1) & \mathrm{N} 4-\mathrm{C} 8 & 1.469(2) \\
\mathrm{S} 1-\mathrm{O} 2 & 1.441(1) & \mathrm{N} 4-\mathrm{C} 7 & 1.471(2) \\
\mathrm{S} 1-\mathrm{O} 1 & 1.445(1) & \mathrm{C} 4-\mathrm{C} 5 & 1.379(2) \\
\mathrm{S} 1-\mathrm{C} 1 & 1.818(2) & \mathrm{C} 5-\mathrm{C} 6 & 1.385(2) \\
\mathrm{F} 1-\mathrm{C} 11 & 1.367(2) & \mathrm{C} 9-\mathrm{C} 10 & 1.475(2) \\
\mathrm{N} 1-\mathrm{O} 4 & 1.218(2) & \mathrm{C} 10-\mathrm{O} 10 & 1.337(2) \\
\mathrm{N} 1-\mathrm{O} 5 & 1.228(2) & \mathrm{O} 10-\mathrm{C} 11 & 1.412(2) \\
\mathrm{N} 1-\mathrm{C} 2 & 1.475(2) & \mathrm{N} 3-\mathrm{O} 8 & 1.224(2) \\
\mathrm{C} 1-\mathrm{C} 6 & 1.389(2) & \mathrm{N} 3-\mathrm{C} 6 & 1.476(2) \\
\mathrm{C} 1-\mathrm{C} 2 & 1.394(2) & \mathrm{N} 4-\mathrm{C} 10 & 1.289(2) \\
\mathrm{N} 2-\mathrm{O} 7 & 1.218(2) & \mathrm{C} 2-\mathrm{C} 3 & 1.384(2) \\
\mathrm{N} 2-\mathrm{O} 6 & 1.229(2) & \mathrm{C} 3-\mathrm{C} 4 & 1.377(2) \\
\mathrm{N} 2-\mathrm{C} 4 & 1.476(2) & \mathrm{N} 3-\mathrm{O} 9 & 1.221(2)
\end{array}
$$

Table S27. Selected bond angles $\left(^{\circ}\right)$ of compound $\mathbf{5 b}$.

$$
\begin{array}{ll|ll}
\mathrm{O} 3-\mathrm{S} 1-\mathrm{O} 2 & 114.5(1) & \mathrm{C} 5-\mathrm{C} 6-\mathrm{C} 1 & 124.0(2) \\
\mathrm{O} 3-\mathrm{S} 1-\mathrm{O} 1 & 115.6(1) & \mathrm{C} 5-\mathrm{C} 6-\mathrm{N} 3 & 115.9(2) \\
& & \mathrm{S} 53 &
\end{array}
$$




\begin{tabular}{ll|ll}
$\mathrm{O} 2-\mathrm{S} 1-\mathrm{O} 1$ & $113.6(1)$ & $\mathrm{C} 1-\mathrm{C} 6-\mathrm{N} 3$ & $120.1(1)$ \\
$\mathrm{O} 3-\mathrm{S} 1-\mathrm{C} 1$ & $104.8(1)$ & $\mathrm{N} 4-\mathrm{C} 10-\mathrm{O} 10$ & $114.4(2)$ \\
$\mathrm{O} 2-\mathrm{S} 1-\mathrm{C} 1$ & $104.3(1)$ & $\mathrm{N} 4-\mathrm{C} 10-\mathrm{C} 9$ & $124.9(2)$ \\
$\mathrm{O} 1-\mathrm{S} 1-\mathrm{C} 1$ & $102.2(1)$ & $\mathrm{O} 10-\mathrm{C} 10-\mathrm{C} 9$ & $120.7(2)$ \\
$\mathrm{O} 4-\mathrm{N} 1-\mathrm{O} 5$ & $125.4(2)$ & $\mathrm{C} 10-\mathrm{O} 10-\mathrm{C} 11$ & $119.8(1)$ \\
$\mathrm{O} 4-\mathrm{N} 1-\mathrm{C} 2$ & $117.8(1)$ & $\mathrm{F} 1-\mathrm{C} 11-\mathrm{O} 10$ & $109.2(1)$ \\
$\mathrm{O} 5-\mathrm{N} 1-\mathrm{C} 2$ & $116.8(1)$ & $\mathrm{O} 9-\mathrm{N} 3-\mathrm{O} 8$ & $125.5(2)$ \\
$\mathrm{C} 6-\mathrm{C} 1-\mathrm{C} 2$ & $115.2(1)$ & $\mathrm{O} 9-\mathrm{N} 3-\mathrm{C} 6$ & $117.0(1)$ \\
$\mathrm{C} 6-\mathrm{C} 1-\mathrm{S} 1$ & $122.8(1)$ & $\mathrm{O} 8-\mathrm{N} 3-\mathrm{C} 6$ & $117.5(1)$ \\
$\mathrm{C} 2-\mathrm{C} 1-\mathrm{S} 1$ & $121.6(1)$ & $\mathrm{C} 10-\mathrm{N} 4-\mathrm{C} 8$ & $122.1(2)$ \\
$\mathrm{O} 7-\mathrm{N} 2-\mathrm{O} 6$ & $124.8(2)$ & $\mathrm{C} 10-\mathrm{N} 4-\mathrm{C} 7$ & $122.9(2)$ \\
$\mathrm{O} 7-\mathrm{N} 2-\mathrm{C} 4$ & $117.9(2)$ & $\mathrm{C} 8-\mathrm{N} 4-\mathrm{C} 7$ & $115.0(2)$ \\
$\mathrm{O} 6-\mathrm{N} 2-\mathrm{C} 4$ & $117.3(2)$ & $\mathrm{C} 3-\mathrm{C} 4-\mathrm{C} 5$ & $123.0(2)$ \\
$\mathrm{C} 3-\mathrm{C} 2-\mathrm{C} 1$ & $123.7(2)$ & $\mathrm{C} 3-\mathrm{C} 4-\mathrm{N} 2$ & $118.4(2)$ \\
$\mathrm{C} 3-\mathrm{C} 2-\mathrm{N} 1$ & $115.8(1)$ & $\mathrm{C} 5-\mathrm{C} 4-\mathrm{N} 2$ & $118.5(2)$ \\
$\mathrm{C} 1-\mathrm{C} 2-\mathrm{N} 1$ & $120.5(1)$ & $\mathrm{C} 4-\mathrm{C} 5-\mathrm{C} 6$ & $116.9(2)$ \\
$\mathrm{C} 4-\mathrm{C} 3-\mathrm{C} 2$ & $117.1(2)$ & &
\end{tabular}

Table S28. Selected torsion angles $\left(^{\circ}\right)$ of compound $\mathbf{5 b}$.

\begin{tabular}{ll|ll}
$\mathrm{O} 3-\mathrm{S} 1-\mathrm{C} 1-\mathrm{C} 6$ & $-97.5(2)$ & $\mathrm{O} 6-\mathrm{N} 2-\mathrm{C} 4-\mathrm{C} 5$ & $-171.1(2)$ \\
$\mathrm{O} 2-\mathrm{S} 1-\mathrm{C} 1-\mathrm{C} 6$ & $23.1(2)$ & $\mathrm{C} 3-\mathrm{C} 4-\mathrm{C} 5-\mathrm{C} 6$ & $-0.2(2)$ \\
$\mathrm{O} 1-\mathrm{S} 1-\mathrm{C} 1-\mathrm{C} 6$ & $141.6(1)$ & $\mathrm{N} 2-\mathrm{C} 4-\mathrm{C} 5-\mathrm{C} 6$ & $-179.6(1)$ \\
$\mathrm{O} 3-\mathrm{S} 1-\mathrm{C} 1-\mathrm{C} 2$ & $90.0(1)$ & $\mathrm{C} 4-\mathrm{C} 5-\mathrm{C} 6-\mathrm{C} 1$ & $-0.6(2)$ \\
$\mathrm{O} 2-\mathrm{S} 1-\mathrm{C} 1-\mathrm{C} 2$ & $-149.3(1)$ & $\mathrm{C} 4-\mathrm{C} 5-\mathrm{C} 6-\mathrm{N} 3$ & $177.6(1)$ \\
$\mathrm{O} 1-\mathrm{S} 1-\mathrm{C} 1-\mathrm{C} 2$ & $-30.9(1)$ & $\mathrm{C} 2-\mathrm{C} 1-\mathrm{C} 6-\mathrm{C} 5$ & $1.3(2)$ \\
$\mathrm{C} 6-\mathrm{C} 1-\mathrm{C} 2-\mathrm{C} 3$ & $-1.2(2)$ & $\mathrm{S} 1-\mathrm{C} 1-\mathrm{C} 6-\mathrm{C} 5$ & $-171.6(1)$ \\
$\mathrm{S} 1-\mathrm{C} 1-\mathrm{C} 2-\mathrm{C} 3$ & $171.8(1)$ & $\mathrm{C} 2-\mathrm{C} 1-\mathrm{C} 6-\mathrm{N} 3$ & $-176.8(1)$ \\
$\mathrm{C} 6-\mathrm{C} 1-\mathrm{C} 2-\mathrm{N} 1$ & $178.5(1)$ & $\mathrm{S} 1-\mathrm{C} 1-\mathrm{C} 6-\mathrm{N} 3$ & $10.3(2)$ \\
$\mathrm{S} 1-\mathrm{C} 1-\mathrm{C} 2-\mathrm{N} 1$ & $-8.5(2)$ & $\mathrm{O} 9-\mathrm{N} 3-\mathrm{C} 6-\mathrm{C} 5$ & $-113.9(2)$ \\
$\mathrm{O} 4-\mathrm{N} 1-\mathrm{C} 2-\mathrm{C} 3$ & $116.8(2)$ & $\mathrm{O} 8-\mathrm{N} 3-\mathrm{C} 6-\mathrm{C} 5$ & $63.9(2)$ \\
$\mathrm{O} 5-\mathrm{N} 1-\mathrm{C} 2-\mathrm{C} 3$ & $-61.4(2)$ & $\mathrm{O} 9-\mathrm{N} 3-\mathrm{C} 6-\mathrm{C} 1$ & $64.3(2)$ \\
$\mathrm{O} 4-\mathrm{N} 1-\mathrm{C} 2-\mathrm{C} 1$ & $-63.0(2)$ & $\mathrm{O} 8-\mathrm{N} 3-\mathrm{C} 6-\mathrm{C} 1$ & $-117.9(2)$ \\
$\mathrm{O} 5-\mathrm{N} 1-\mathrm{C} 2-\mathrm{C} 1$ & $118.9(2)$ & $\mathrm{C} 8-\mathrm{N} 4-\mathrm{C} 10-\mathrm{O} 10$ & $0.7(2)$ \\
$\mathrm{C} 1-\mathrm{C} 2-\mathrm{C} 3-\mathrm{C} 4$ & $0.5(2)$ & $\mathrm{C} 7-\mathrm{N} 4-\mathrm{C} 10-\mathrm{O} 10$ & $-178.5(2)$ \\
$\mathrm{N} 1-\mathrm{C} 2-\mathrm{C} 3-\mathrm{C} 4$ & $-179.2(1)$ & $\mathrm{C} 8-\mathrm{N} 4-\mathrm{C} 10-\mathrm{C} 9$ & $-179.9(2)$ \\
$\mathrm{C} 2-\mathrm{C} 3-\mathrm{C} 4-\mathrm{C} 5$ & $0.2(2)$ & $\mathrm{C} 7-\mathrm{N} 4-\mathrm{C} 10-\mathrm{C} 9$ & $0.9(3)$ \\
$\mathrm{C} 2-\mathrm{C} 3-\mathrm{C} 4-\mathrm{N} 2$ & $179.7(1)$ & $\mathrm{N} 4-\mathrm{C} 10-\mathrm{O} 10-\mathrm{C} 11$ & $-165.9(1)$ \\
$\mathrm{O} 7-\mathrm{N} 2-\mathrm{C} 4-\mathrm{C} 3$ & $-170.9(2)$ & $\mathrm{C} 9-\mathrm{C} 10-\mathrm{O} 10-\mathrm{C} 11$ & $14.6(2)$ \\
$\mathrm{O} 6-\mathrm{N} 2-\mathrm{C} 4-\mathrm{C} 3$ & $9.4(2)$ & $\mathrm{C} 10-\mathrm{O} 10-\mathrm{C} 11-\mathrm{F} 1$ & $-80.5(2)$
\end{tabular}




$$
\mathrm{O} 7-\mathrm{N} 2-\mathrm{C} 4-\mathrm{C} 5 \quad 8.6(2)
$$

\subsection{Compound $\mathbf{5 c}$}

Table S29. Details for X-ray data collection and structure refinement for compound 5c.

\begin{tabular}{ll}
\hline & $\mathbf{5 c}$ \\
\hline CCDC & 2025117 \\
& S55
\end{tabular}




\begin{tabular}{|c|c|}
\hline Empirical formula & $\mathrm{C}_{10} \mathrm{H}_{11} \mathrm{FN}_{4} \mathrm{O}_{10} \mathrm{~S}$ \\
\hline Formula mass & 398.29 \\
\hline $\mathrm{T}[\mathrm{K}]$ & $143(2)$ \\
\hline Crystal size $[\mathrm{mm}]$ & $0.183 \times 0.118 \times 0.079$ \\
\hline Crystal description & colorless platelet \\
\hline Crystal system & monoclinic \\
\hline Space group & $C 2 / c$ \\
\hline $\mathrm{a}[\AA \hat{A}]$ & $24.606(3)$ \\
\hline 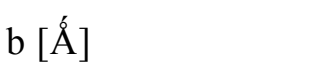 & $8.2530(5)$ \\
\hline c [Á́] & $16.5340(14)$ \\
\hline$\alpha\left[^{\circ}\right]$ & 90.0 \\
\hline$\beta\left[^{\circ}\right]$ & $111.874(10)$ \\
\hline$\gamma\left[{ }^{\circ}\right]$ & 90.0 \\
\hline $\mathrm{V}\left[\AA^{3}\right]$ & $3115.9(5)$ \\
\hline $\mathrm{Z}$ & 8 \\
\hline$\rho_{\text {calcd. }}\left[\mathrm{g} \mathrm{cm}^{-3}\right]$ & 1.698 \\
\hline$\mu\left[\mathrm{mm}^{-1}\right]$ & 0.285 \\
\hline$F(000)$ & 1632 \\
\hline$\Theta$ range $\left[{ }^{\circ}\right]$ & $4.21-25.24$ \\
\hline \multirow[t]{3}{*}{ Index ranges } & $-29 \leq h \leq 32$ \\
\hline & $-10 \leq k \leq 10$ \\
\hline & $-22 \leq l \leq 21$ \\
\hline Reflns. collected & 13545 \\
\hline Reflns. obsd. & 2297 \\
\hline Reflns. unique & $\begin{array}{l}3840 \\
\left(\mathrm{R}_{\mathrm{int}}=0.0785\right)\end{array}$ \\
\hline$R_{1}, w R_{2}(2 \sigma$ data $)$ & $0.0527,0.0955$ \\
\hline$R_{1}, w R_{2}$ (all data) & $0.1064,0.1175$ \\
\hline GOOF on $F^{2}$ & 1.011 \\
\hline Peak/hole [e $\AA^{-3}$ ] & $0.331 /-0.363$ \\
\hline
\end{tabular}



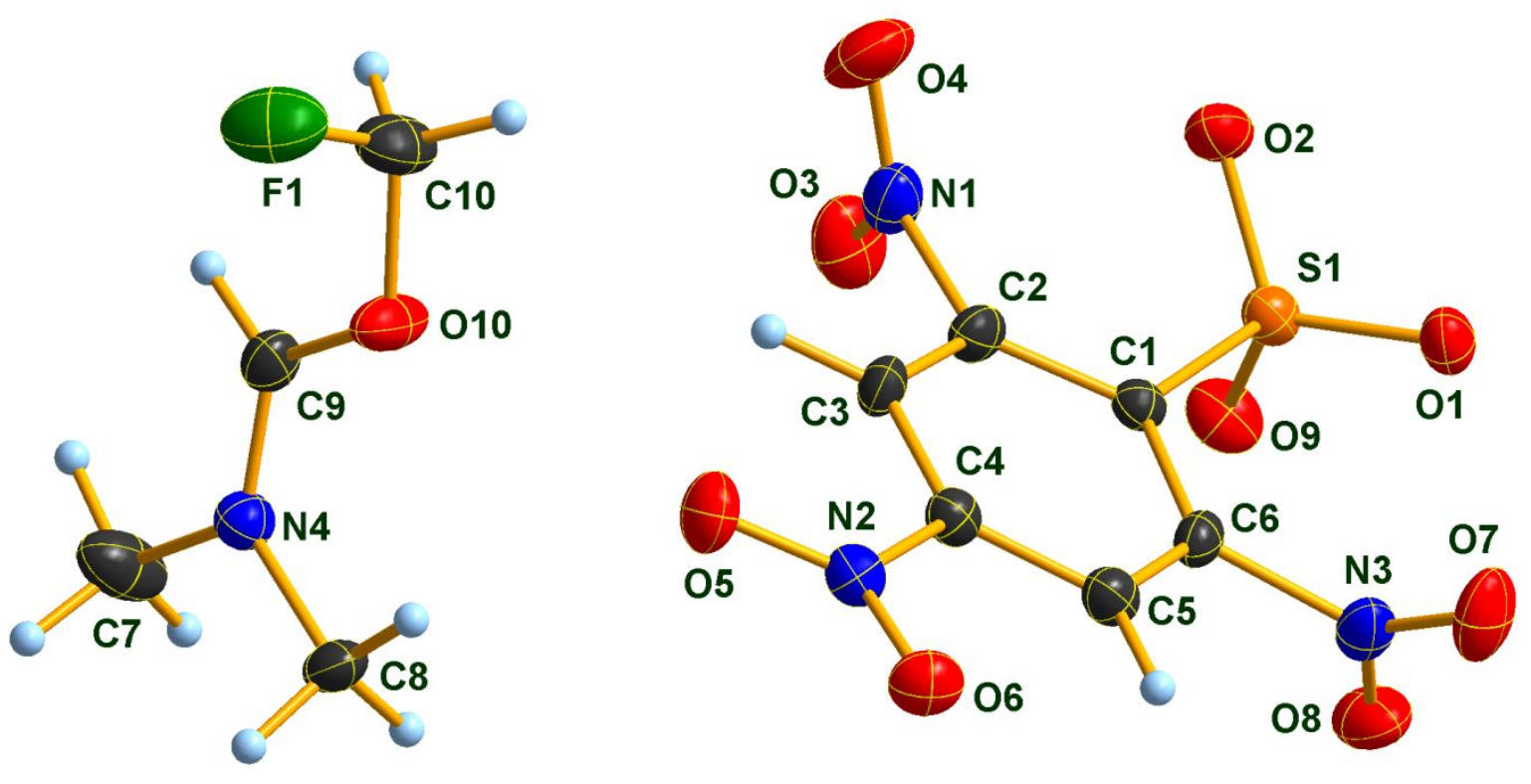

Figure S8. Molecular structure of compound 5c in the crystal. DIAMOND ${ }^{\mathrm{e})}$ representation; thermal ellipsoids are drawn at $50 \%$ probability level.

Table S30. Selected bond lengths $(\AA)$ of compound 5c.

\begin{tabular}{ll|ll}
$\mathrm{S} 1-\mathrm{O} 9$ & $1.438(2)$ & $\mathrm{C} 3-\mathrm{C} 2$ & $1.382(4)$ \\
$\mathrm{S} 1-\mathrm{O} 1$ & $1.441(2)$ & $\mathrm{C} 5-\mathrm{C} 4$ & $1.375(4)$ \\
$\mathrm{S} 1-\mathrm{O} 2$ & $1.450(2)$ & $\mathrm{O} 7-\mathrm{N} 3$ & $1.223(3)$ \\
$\mathrm{S} 1-\mathrm{C} 1$ & $1.807(3)$ & $\mathrm{O} 8-\mathrm{N} 3$ & $1.224(3)$ \\
$\mathrm{O} 6-\mathrm{N} 2$ & $1.227(3)$ & $\mathrm{C} 9-\mathrm{O} 10$ & $1.317(3)$ \\
$\mathrm{C} 6-\mathrm{C} 5$ & $1.382(4)$ & $\mathrm{O} 10-\mathrm{C} 10$ & $1.423(3)$ \\
$\mathrm{C} 6-\mathrm{C} 1$ & $1.393(3)$ & $\mathrm{F} 1-\mathrm{C} 10$ & $1.349(4)$ \\
$\mathrm{C} 6-\mathrm{N} 3$ & $1.474(3)$ & $\mathrm{N} 2-\mathrm{C} 4$ & $1.468(3)$ \\
$\mathrm{N} 1-\mathrm{O} 3$ & $1.219(3)$ & $\mathrm{N} 4-\mathrm{C} 9$ & $1.268(3)$ \\
$\mathrm{N} 1-\mathrm{O} 4$ & $1.222(3)$ & $\mathrm{N} 4-\mathrm{C} 8$ & $1.464(3)$ \\
$\mathrm{N} 1-\mathrm{C} 2$ & $1.481(3)$ & $\mathrm{N} 4-\mathrm{C} 7$ & $1.464(3)$ \\
$\mathrm{C} 1-\mathrm{C} 2$ & $1.399(4)$ & $\mathrm{C} 3-\mathrm{C} 4$ & $1.376(4)$ \\
$\mathrm{N} 2-\mathrm{O} 5$ & $1.223(3)$ & &
\end{tabular}

Table S31. Selected bond angles $\left(^{\circ}\right)$ of compound $\mathbf{5 c}$.

$$
\begin{array}{ll|ll}
\mathrm{O} 9-\mathrm{S} 1-\mathrm{O} 1 & 115.4(1) & \mathrm{C} 3-\mathrm{C} 4-\mathrm{N} 2 & 118.4(2) \\
\mathrm{O} 9-\mathrm{S} 1-\mathrm{O} 2 & 114.5(1) & \mathrm{C} 3-\mathrm{C} 2-\mathrm{C} 1 & 124.0(2)
\end{array}
$$




\begin{tabular}{ll|ll}
$\mathrm{O} 1-\mathrm{S} 1-\mathrm{O} 2$ & $113.2(1)$ & $\mathrm{C} 3-\mathrm{C} 2-\mathrm{N} 1$ & $115.9(2)$ \\
$\mathrm{O} 9-\mathrm{S} 1-\mathrm{C} 1$ & $105.3(1)$ & $\mathrm{C} 1-\mathrm{C} 2-\mathrm{N} 1$ & $120.1(2)$ \\
$\mathrm{O} 1-\mathrm{S} 1-\mathrm{C} 1$ & $102.9(1)$ & $\mathrm{O} 7-\mathrm{N} 3-\mathrm{O} 8$ & $125.3(2)$ \\
$\mathrm{O} 2-\mathrm{S} 1-\mathrm{C} 1$ & $103.7(1)$ & $\mathrm{O} 7-\mathrm{N} 3-\mathrm{C} 6$ & $117.4(2)$ \\
$\mathrm{C} 5-\mathrm{C} 6-\mathrm{C} 1$ & $124.0(2)$ & $\mathrm{O} 8-\mathrm{N} 3-\mathrm{C} 6$ & $117.2(2)$ \\
$\mathrm{C} 5-\mathrm{C} 6-\mathrm{N} 3$ & $115.7(2)$ & $\mathrm{N} 4-\mathrm{C} 9-\mathrm{O} 10$ & $118.8(3)$ \\
$\mathrm{C} 1-\mathrm{C} 6-\mathrm{N} 3$ & $120.2(2)$ & $\mathrm{C} 9-\mathrm{O} 10-\mathrm{C} 10$ & $116.3(2)$ \\
$\mathrm{O} 3-\mathrm{N} 1-\mathrm{O} 4$ & $125.6(3)$ & $\mathrm{F} 1-\mathrm{C} 10-\mathrm{O} 10$ & $107.2(3)$ \\
$\mathrm{O} 3-\mathrm{N} 1-\mathrm{C} 2$ & $117.3(2)$ & $\mathrm{C} 9-\mathrm{N} 4-\mathrm{C} 8$ & $123.1(2)$ \\
$\mathrm{O} 4-\mathrm{N} 1-\mathrm{C} 2$ & $117.1(2)$ & $\mathrm{C} 9-\mathrm{N} 4-\mathrm{C} 7$ & $119.7(2)$ \\
$\mathrm{C} 6-\mathrm{C} 1-\mathrm{C} 2$ & $114.6(2)$ & $\mathrm{C} 8-\mathrm{N} 4-\mathrm{C} 7$ & $117.1(2)$ \\
$\mathrm{C} 6-\mathrm{C} 1-\mathrm{S} 1$ & $122.4(2)$ & $\mathrm{C} 4-\mathrm{C} 3-\mathrm{C} 2$ & $117.3(2)$ \\
$\mathrm{C} 2-\mathrm{C} 1-\mathrm{S} 1$ & $122.7(2)$ & $\mathrm{C} 4-\mathrm{C} 5-\mathrm{C} 6$ & $117.4(3)$ \\
$\mathrm{O} 5-\mathrm{N} 2-\mathrm{O} 6$ & $124.7(2)$ & $\mathrm{C} 5-\mathrm{C} 4-\mathrm{C} 3$ & $122.6(3)$ \\
$\mathrm{O} 5-\mathrm{N} 2-\mathrm{C} 4$ & $117.7(2)$ & $\mathrm{C} 5-\mathrm{C} 4-\mathrm{N} 2$ & $119.0(2)$ \\
$\mathrm{O} 6-\mathrm{N} 2-\mathrm{C} 4$ & $117.6(2)$ & &
\end{tabular}

Table S32. Selected torsion angles $\left(^{\circ}\right)$ of compound $\mathbf{5 c}$.

\begin{tabular}{ll|ll}
$\mathrm{C} 5-\mathrm{C} 6-\mathrm{C} 1-\mathrm{C} 2$ & $-1.9(4)$ & $\mathrm{O} 6-\mathrm{N} 2-\mathrm{C} 4-\mathrm{C} 3$ & $-174.9(2)$ \\
$\mathrm{N} 3-\mathrm{C} 6-\mathrm{C} 1-\mathrm{C} 2$ & $178.1(2)$ & $\mathrm{C} 4-\mathrm{C} 3-\mathrm{C} 2-\mathrm{C} 1$ & $-0.6(4)$ \\
$\mathrm{C} 5-\mathrm{C} 6-\mathrm{C} 1-\mathrm{S} 1$ & $172.2(2)$ & $\mathrm{C} 4-\mathrm{C} 3-\mathrm{C} 2-\mathrm{N} 1$ & $177.2(2)$ \\
$\mathrm{N} 3-\mathrm{C} 6-\mathrm{C} 1-\mathrm{S} 1$ & $-7.9(3)$ & $\mathrm{C} 6-\mathrm{C} 1-\mathrm{C} 2-\mathrm{C} 3$ & $1.7(4)$ \\
$\mathrm{O} 9-\mathrm{S} 1-\mathrm{C} 1-\mathrm{C} 6$ & $92.4(2)$ & $\mathrm{S} 1-\mathrm{C} 1-\mathrm{C} 2-\mathrm{C} 3$ & $-172.4(2)$ \\
$\mathrm{O} 1-\mathrm{S} 1-\mathrm{C} 1-\mathrm{C} 6$ & $-28.8(2)$ & $\mathrm{C} 6-\mathrm{C} 1-\mathrm{C} 2-\mathrm{N} 1$ & $-176.0(2)$ \\
$\mathrm{O} 2-\mathrm{S} 1-\mathrm{C} 1-\mathrm{C} 6$ & $-147.0(2)$ & $\mathrm{S} 1-\mathrm{C} 1-\mathrm{C} 2-\mathrm{N} 1$ & $10.0(3)$ \\
$\mathrm{O} 9-\mathrm{S} 1-\mathrm{C} 1-\mathrm{C} 2$ & $-94.0(2)$ & $\mathrm{O} 3-\mathrm{N} 1-\mathrm{C} 2-\mathrm{C} 3$ & $-113.6(3)$ \\
$\mathrm{O} 1-\mathrm{S} 1-\mathrm{C} 1-\mathrm{C} 2$ & $144.7(2)$ & $\mathrm{O} 4-\mathrm{N} 1-\mathrm{C} 2-\mathrm{C} 3$ & $64.5(3)$ \\
$\mathrm{O} 2-\mathrm{S} 1-\mathrm{C} 1-\mathrm{C} 2$ & $26.6(2)$ & $\mathrm{O} 3-\mathrm{N} 1-\mathrm{C} 2-\mathrm{C} 1$ & $64.2(3)$ \\
$\mathrm{C} 1-\mathrm{C} 6-\mathrm{C} 5-\mathrm{C} 4$ & $1.0(4)$ & $\mathrm{O} 4-\mathrm{N} 1-\mathrm{C} 2-\mathrm{C} 1$ & $-117.6(3)$ \\
$\mathrm{N} 3-\mathrm{C} 6-\mathrm{C} 5-\mathrm{C} 4$ & $-179.0(2)$ & $\mathrm{C} 5-\mathrm{C} 6-\mathrm{N} 3-\mathrm{O} 7$ & $-63.6(3)$ \\
$\mathrm{C} 6-\mathrm{C} 5-\mathrm{C} 4-\mathrm{C} 3$ & $0.3(4)$ & $\mathrm{C} 1-\mathrm{C} 6-\mathrm{N} 3-\mathrm{O} 7$ & $116.4(3)$ \\
$\mathrm{C} 6-\mathrm{C} 5-\mathrm{C} 4-\mathrm{N} 2$ & $179.8(2)$ & $\mathrm{C} 5-\mathrm{C} 6-\mathrm{N} 3-\mathrm{O} 8$ & $114.0(3)$ \\
$\mathrm{C} 2-\mathrm{C} 3-\mathrm{C} 4-\mathrm{C} 5$ & $-0.5(4)$ & $\mathrm{C} 1-\mathrm{C} 6-\mathrm{N} 3-\mathrm{O} 8$ & $-66.0(3)$ \\
$\mathrm{C} 2-\mathrm{C} 3-\mathrm{C} 4-\mathrm{N} 2$ & $-180.0(2)$ & $\mathrm{C} 8-\mathrm{N} 4-\mathrm{C} 9-\mathrm{O} 10$ & $2.2(4)$ \\
$\mathrm{O} 5-\mathrm{N} 2-\mathrm{C} 4-\mathrm{C} 5$ & $-174.6(2)$ & $\mathrm{C} 7-\mathrm{N} 4-\mathrm{C} 9-\mathrm{O} 10$ & $-175.9(3)$ \\
$\mathrm{O} 6-\mathrm{N} 2-\mathrm{C} 4-\mathrm{C} 5$ & $5.6(3)$ & $\mathrm{N} 4-\mathrm{C} 9-\mathrm{O} 10-\mathrm{C} 10$ & $-171.5(3)$ \\
$\mathrm{O} 5-\mathrm{N} 2-\mathrm{C} 4-\mathrm{C} 3$ & $4.9(3)$ & $\mathrm{C} 9-\mathrm{O} 10-\mathrm{C} 10-\mathrm{F} 1$ & $84.8(3)$
\end{tabular}




\subsection{Compound $\mathbf{6 b}$}

Table S33. Details for X-ray data collection and structure refinement for compound $\mathbf{6 b}$.

\begin{tabular}{|c|c|}
\hline & $6 \mathbf{b}$ \\
\hline $\mathrm{CCDC}$ & 2025108 \\
\hline Empirical formula & $\mathrm{C}_{8} \mathrm{H}_{10} \mathrm{~N}_{4} \mathrm{O}_{9} \mathrm{~S}$ \\
\hline Formula mass & 338.26 \\
\hline $\mathrm{T}[\mathrm{K}]$ & $143(2)$ \\
\hline Crystal size $[\mathrm{mm}]$ & $0.25 \times 0.15 \times 0.10$ \\
\hline Crystal description & colorless block \\
\hline Crystal system & monoclinic \\
\hline Space group & $P 21 / c$ \\
\hline 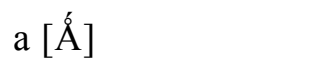 & $11.0816(5)$ \\
\hline $\mathrm{b}[\AA ̊ d]$ & $13.3769(5)$ \\
\hline c [Á] & $9.1004(4)$ \\
\hline$\alpha\left[^{\circ}\right]$ & 90.0 \\
\hline$\beta\left[^{\circ}\right]$ & $95.478(4)$ \\
\hline$\gamma\left[^{\circ}\right]$ & 90.0 \\
\hline $\mathrm{V}\left[\AA^{3}\right]$ & $1342.86(10)$ \\
\hline $\mathrm{Z}$ & 4 \\
\hline$\rho_{\text {calcd. }}\left[\mathrm{g} \mathrm{cm}^{-3}\right]$ & 1.673 \\
\hline$\mu\left[\mathrm{mm}^{-1}\right]$ & 0.298 \\
\hline$F(000)$ & 696 \\
\hline$\Theta$ range $\left[{ }^{\circ}\right]$ & $4.31-25.24$ \\
\hline \multirow[t]{3}{*}{ Index ranges } & $-13 \leq h \leq 13$ \\
\hline & $-16 \leq k \leq 16$ \\
\hline & $-9 \leq l \leq 11$ \\
\hline Reflns. collected & 10282 \\
\hline Reflns. obsd. & 1962 \\
\hline Reflns. unique & $\begin{array}{l}2733 \\
\left(\mathrm{R}_{\mathrm{int}}=0.0602\right)\end{array}$ \\
\hline$R_{1}, w R_{2}(2 \sigma$ data $)$ & $0.0424,0.0802$ \\
\hline$R_{1}, w R_{2}$ (all data) & $0.0683,0.0914$ \\
\hline GOOF on $F^{2}$ & 1.032 \\
\hline Peak/hole $\left[\mathrm{e} \AA^{-3}\right]$ & $0.377 /-0.430$ \\
\hline
\end{tabular}



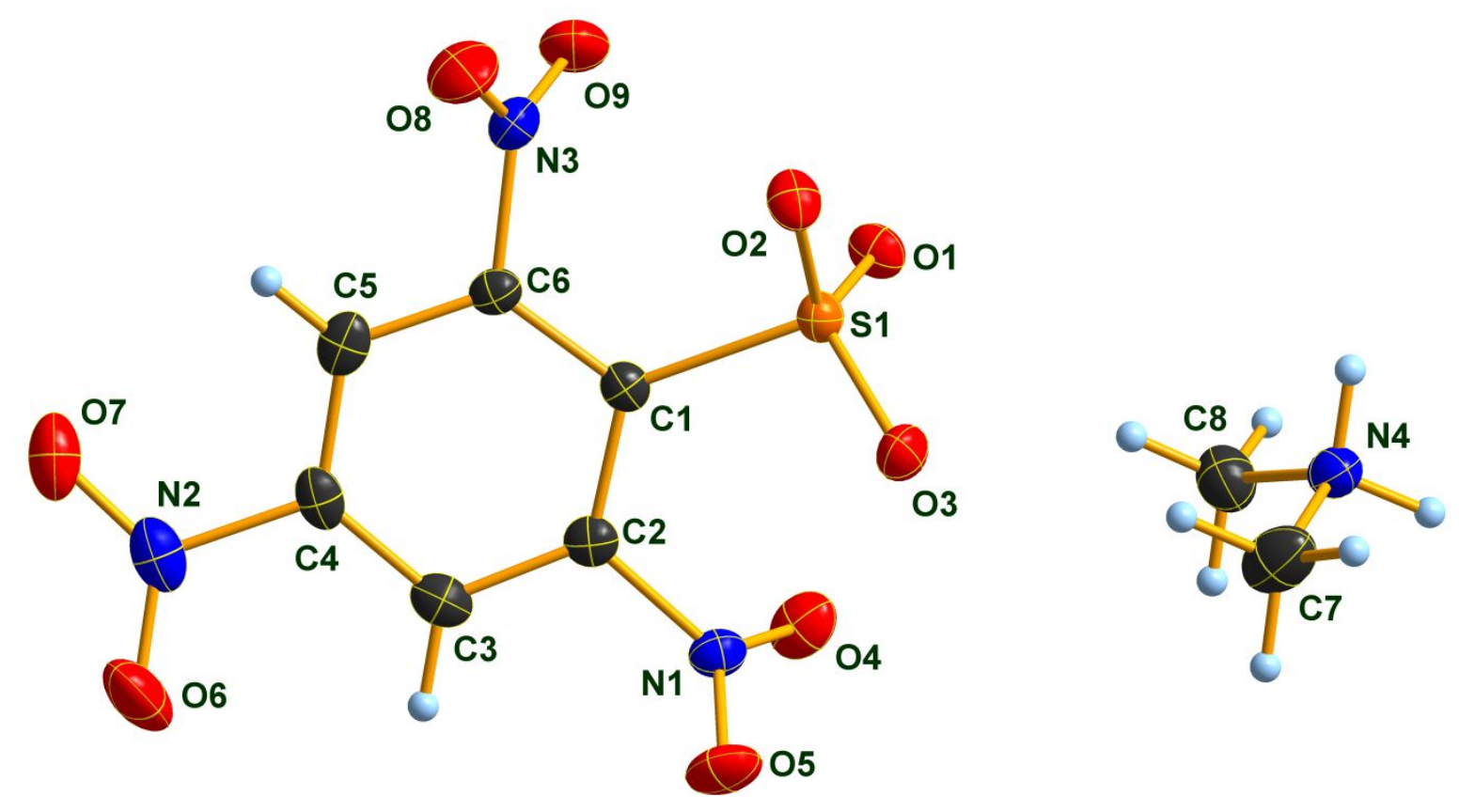

Figure S9. Molecular structure of compound $\mathbf{6 b}$ in the crystal. DIAMOND ${ }^{\mathrm{e})}$ representation; thermal ellipsoids are drawn at $50 \%$ probability level.

Table S34. Selected bond lengths ( $\AA$ ) of compound $\mathbf{6 b}$.

$$
\begin{array}{ll|ll}
\mathrm{S} 1-\mathrm{O} 3 & 1.437(2) & \mathrm{C} 5-\mathrm{C} 4 & 1.376(3) \\
\mathrm{S} 1-\mathrm{O} 1 & 1.445(2) & \mathrm{C} 4-\mathrm{C} 3 & 1.378(3) \\
\mathrm{S} 1-\mathrm{O} 2 & 1.449(2) & \mathrm{N} 4-\mathrm{C} 7 & 1.476(3) \\
\mathrm{S} 1-\mathrm{C} 1 & 1.812(2) & \mathrm{N} 4-\mathrm{C} 8 & 1.480(3) \\
\mathrm{N} 1-\mathrm{O} 5 & 1.217(2) & \mathrm{N} 3-\mathrm{O} 8 & 1.218(2) \\
\mathrm{N} 1-\mathrm{O} 4 & 1.225(2) & \mathrm{N} 2-\mathrm{O} 6 & 1.223(3) \\
\mathrm{N} 1-\mathrm{C} 2 & 1.476(3) & \mathrm{N} 2-\mathrm{O} 7 & 1.223(3) \\
\mathrm{C} 1-\mathrm{C} 2 & 1.392(3) & \mathrm{N} 2-\mathrm{C} 4 & 1.472(3) \\
\mathrm{C} 1-\mathrm{C} 6 & 1.393(3) & \mathrm{C} 6-\mathrm{C} 5 & 1.376(3) \\
\mathrm{O} 9-\mathrm{N} 3 & 1.231(3) & \mathrm{C} 6-\mathrm{N} 3 & 1.478(3) \\
\mathrm{C} 2-\mathrm{C} 3 & 1.383(3) & &
\end{array}
$$

Table S35. Selected bond angles $\left(^{\circ}\right)$ of compound $\mathbf{6 b}$.

$$
\begin{array}{ll|ll}
\mathrm{O} 3-\mathrm{S} 1-\mathrm{O} 1 & 115.2(1) & \mathrm{C} 3-\mathrm{C} 4-\mathrm{N} 2 & 119.6(2) \\
\mathrm{O} 3-\mathrm{S} 1-\mathrm{O} 2 & 114.4(1) & \mathrm{C} 7-\mathrm{N} 4-\mathrm{C} 8 & 113.4(2) \\
\mathrm{O} 1-\mathrm{S} 1-\mathrm{O} 2 & 112.8(1) & \mathrm{C} 4-\mathrm{C} 3-\mathrm{C} 2 & 117.4(2)
\end{array}
$$




$$
\begin{array}{ll|ll}
\mathrm{O} 3-\mathrm{S} 1-\mathrm{C} 1 & 104.3(1) & \mathrm{O} 8-\mathrm{N} 3-\mathrm{O} 9 & 125.7(2) \\
\mathrm{O} 1-\mathrm{S} 1-\mathrm{C} 1 & 105.6(1) & \mathrm{O} 8-\mathrm{N} 3-\mathrm{C} 6 & 118.1(2) \\
\mathrm{O} 2-\mathrm{S} 1-\mathrm{C} 1 & 102.9(1) & \mathrm{O} 9-\mathrm{N} 3-\mathrm{C} 6 & 116.1(2) \\
\mathrm{O} 5-\mathrm{N} 1-\mathrm{O} 4 & 125.3(2) & \mathrm{O} 6-\mathrm{N} 2-\mathrm{O} 7 & 125.0(2) \\
\mathrm{O} 5-\mathrm{N} 1-\mathrm{C} 2 & 117.5(2) & \mathrm{O} 6-\mathrm{N} 2-\mathrm{C} 4 & 117.6(2) \\
\mathrm{O} 4-\mathrm{N} 1-\mathrm{C} 2 & 117.1(2) & \mathrm{O} 7-\mathrm{N} 2-\mathrm{C} 4 & 117.4(2) \\
\mathrm{C} 2-\mathrm{C} 1-\mathrm{C} 6 & 114.9(2) & \mathrm{C} 5-\mathrm{C} 6-\mathrm{C} 1 & 124.0(2) \\
\mathrm{C} 2-\mathrm{C} 1-\mathrm{S} 1 & 123.4(2) & \mathrm{C} 5-\mathrm{C} 6-\mathrm{N} 3 & 115.3(2) \\
\mathrm{C} 6-\mathrm{C} 1-\mathrm{S} 1 & 121.1(2) & \mathrm{C} 1-\mathrm{C} 6-\mathrm{N} 3 & 120.7(2) \\
\mathrm{C} 3-\mathrm{C} 2-\mathrm{C} 1 & 123.8(2) & \mathrm{C} 6-\mathrm{C} 5-\mathrm{C} 4 & 117.6(2) \\
\mathrm{C} 3-\mathrm{C} 2-\mathrm{N} 1 & 115.6(2) & \mathrm{C} 5-\mathrm{C} 4-\mathrm{C} 3 & 122.3(2) \\
\mathrm{C} 1-\mathrm{C} 2-\mathrm{N} 1 & 120.6(2) & \mathrm{C} 5-\mathrm{C} 4-\mathrm{N} 2 & 118.1(2)
\end{array}
$$

Table S36. Selected torsion angles $\left(^{\circ}\right)$ of compound $\mathbf{6 b}$.

$$
\begin{array}{ll|ll}
\mathrm{O} 3-\mathrm{S} 1-\mathrm{C} 1-\mathrm{C} 2 & -24.0(2) & \mathrm{S} 1-\mathrm{C} 1-\mathrm{C} 6-\mathrm{N} 3 & 11.0(3) \\
\mathrm{O} 1-\mathrm{S} 1-\mathrm{C} 1-\mathrm{C} 2 & 97.9(2) & \mathrm{C} 1-\mathrm{C} 6-\mathrm{C} 5-\mathrm{C} 4 & -1.4(4) \\
\mathrm{O} 2-\mathrm{S} 1-\mathrm{C} 1-\mathrm{C} 2 & -143.7(2) & \mathrm{N} 3-\mathrm{C} 6-\mathrm{C} 5-\mathrm{C} 4 & 177.7(2) \\
\mathrm{O} 3-\mathrm{S} 1-\mathrm{C} 1-\mathrm{C} 6 & 146.8(2) & \mathrm{C} 6-\mathrm{C} 5-\mathrm{C} 4-\mathrm{C} 3 & 0.9(3) \\
\mathrm{O} 1-\mathrm{S} 1-\mathrm{C} 1-\mathrm{C} 6 & -91.4(2) & \mathrm{C} 6-\mathrm{C} 5-\mathrm{C} 4-\mathrm{N} 2 & -179.0(2) \\
\mathrm{O} 2-\mathrm{S} 1-\mathrm{C} 1-\mathrm{C} 6 & 27.1(2) & \mathrm{O} 6-\mathrm{N} 2-\mathrm{C} 4-\mathrm{C} 5 & -169.1(2) \\
\mathrm{C} 6-\mathrm{C} 1-\mathrm{C} 2-\mathrm{C} 3 & -1.3(3) & \mathrm{O} 7-\mathrm{N} 2-\mathrm{C} 4-\mathrm{C} 5 & 11.2(3) \\
\mathrm{S} 1-\mathrm{C} 1-\mathrm{C} 2-\mathrm{C} 3 & 170.0(2) & \mathrm{O} 6-\mathrm{N} 2-\mathrm{C} 4-\mathrm{C} 3 & 11.1(3) \\
\mathrm{C} 6-\mathrm{C} 1-\mathrm{C} 2-\mathrm{N} 1 & 177.9(2) & \mathrm{O} 7-\mathrm{N} 2-\mathrm{C} 4-\mathrm{C} 3 & -168.7(2) \\
\mathrm{S} 1-\mathrm{C} 1-\mathrm{C} 2-\mathrm{N} 1 & -10.8(3) & \mathrm{C} 5-\mathrm{C} 4-\mathrm{C} 3-\mathrm{C} 2 & -0.6(3) \\
\mathrm{O} 5-\mathrm{N} 1-\mathrm{C} 2-\mathrm{C} 3 & -57.7(3) & \mathrm{N} 2-\mathrm{C} 4-\mathrm{C} 3-\mathrm{C} 2 & 179.2(2) \\
\mathrm{O} 4-\mathrm{N} 1-\mathrm{C} 2-\mathrm{C} 3 & 118.6(2) & \mathrm{C} 1-\mathrm{C} 2-\mathrm{C} 3-\mathrm{C} 4 & 0.9(3) \\
\mathrm{O} 5-\mathrm{N} 1-\mathrm{C} 2-\mathrm{C} 1 & 123.1(2) & \mathrm{N} 1-\mathrm{C} 2-\mathrm{C} 3-\mathrm{C} 4 & -178.4(2) \\
\mathrm{O} 4-\mathrm{N} 1-\mathrm{C} 2-\mathrm{C} 1 & -60.6(3) & \mathrm{C} 5-\mathrm{C} 6-\mathrm{N} 3-\mathrm{O} 8 & 60.9(3) \\
\mathrm{C} 2-\mathrm{C} 1-\mathrm{C} 6-\mathrm{C} 5 & 1.5(3) & \mathrm{C} 1-\mathrm{C} 6-\mathrm{N} 3-\mathrm{O} 8 & -120.0(2) \\
\mathrm{S} 1-\mathrm{C} 1-\mathrm{C} 6-\mathrm{C} 5 & -170.0(2) & \mathrm{C} 5-\mathrm{C} 6-\mathrm{N} 3-\mathrm{O} 9 & -115.7(2) \\
\mathrm{C} 2-\mathrm{C} 1-\mathrm{C} 6-\mathrm{N} 3 & -177.5(2) & \mathrm{C} 1-\mathrm{C} 6-\mathrm{N} 3-\mathrm{O} 9 & 63.4(3)
\end{array}
$$

\subsection{Compound $7 \mathbf{b}$}

Table S37. Details for X-ray data collection and structure refinement for compound $\mathbf{7 b}$. 


\begin{tabular}{ll}
\hline & $7 \mathbf{b}$ \\
\hline CCDC & 2025118 \\
Empirical formula & $\mathrm{C}_{25} \mathrm{H}_{19} \mathrm{FN}_{3} \mathrm{O}_{9} \mathrm{PS}_{2}$ \\
Formula mass & 619.52 \\
$\mathrm{~T}[\mathrm{~K}]$ & $143(2)$ \\
Crystal size [mm] & $0.25 \times 0.06 \times 0.06$ \\
Crystal description & colorless rod \\
Crystal system & monoclinic \\
Space group & $P 21 / c$ \\
$\mathrm{a}[\AA \AA$ & \\
$\mathrm{b}[\AA ̊$ &
\end{tabular}



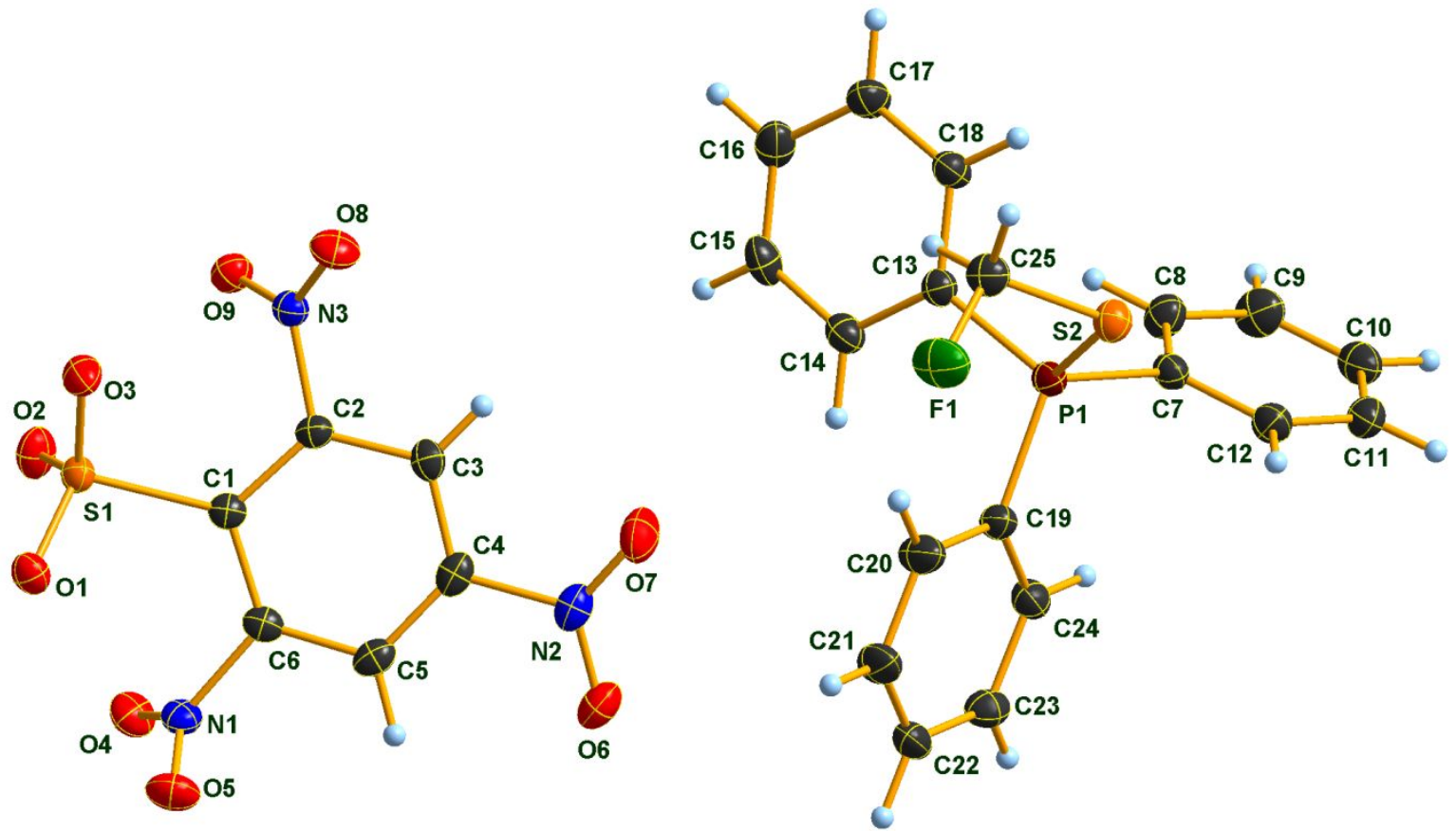

Figure S10. Molecular structure of compound $\mathbf{7 b}$ in the crystal. DIAMOND ${ }^{\mathrm{e})}$ representation; thermal ellipsoids are drawn at $50 \%$ probability level.

Table S38. Selected bond lengths $(\AA)$ of compound $\mathbf{7 b}$.

\begin{tabular}{ll|ll}
$\mathrm{S} 1-\mathrm{O} 2$ & $1.437(2)$ & $\mathrm{C} 5-\mathrm{C} 6$ & $1.375(3)$ \\
$\mathrm{S} 1-\mathrm{O} 1$ & $1.445(1)$ & $\mathrm{C} 7-\mathrm{C} 8$ & $1.389(3)$ \\
$\mathrm{S} 1-\mathrm{O} 3$ & $1.446(1)$ & $\mathrm{C} 7-\mathrm{C} 12$ & $1.398(3)$ \\
$\mathrm{S} 1-\mathrm{C} 1$ & $1.821(2)$ & $\mathrm{C} 23-\mathrm{C} 22$ & $1.377(3)$ \\
$\mathrm{P} 1-\mathrm{C} 13$ & $1.784(2)$ & $\mathrm{C} 23-\mathrm{C} 24$ & $1.387(3)$ \\
$\mathrm{P} 1-\mathrm{C} 7$ & $1.786(2)$ & $\mathrm{C} 15-\mathrm{C} 16$ & $1.381(3)$ \\
$\mathrm{P} 1-\mathrm{C} 19$ & $1.791(2)$ & $\mathrm{C} 15-\mathrm{C} 14$ & $1.392(3)$ \\
$\mathrm{P} 1-\mathrm{S} 2$ & $2.076(1)$ & $\mathrm{C} 14-\mathrm{C} 13$ & $1.397(3)$ \\
$\mathrm{N} 1-\mathrm{O} 5$ & $1.219(2)$ & $\mathrm{C} 13-\mathrm{C} 18$ & $1.401(3)$ \\
$\mathrm{N} 1-\mathrm{O} 4$ & $1.224(2)$ & $\mathrm{C} 19-\mathrm{C} 24$ & $1.390(3)$ \\
$\mathrm{N} 1-\mathrm{C} 6$ & $1.477(2)$ & $\mathrm{C} 19-\mathrm{C} 20$ & $1.394(3)$ \\
$\mathrm{F} 1-\mathrm{C} 25$ & $1.378(2)$ & $\mathrm{C} 18-\mathrm{C} 17$ & $1.383(3)$ \\
$\mathrm{C} 1-\mathrm{C} 2$ & $1.392(2)$ & $\mathrm{C} 22-\mathrm{C} 21$ & $1.381(3)$ \\
$\mathrm{C} 1-\mathrm{C} 6$ & $1.397(2)$ & $\mathrm{C} 21-\mathrm{C} 20$ & $1.381(3)$ \\
$\mathrm{C} 11-\mathrm{C} 12$ & $1.381(3)$ & $\mathrm{C} 17-\mathrm{C} 16$ & $1.391(3)$ \\
$\mathrm{C} 11-\mathrm{C} 10$ & $1.382(3)$ & $\mathrm{C} 3-\mathrm{C} 4$ & $1.379(3)$ \\
$\mathrm{C} 9-\mathrm{C} 10$ & $1.384(3)$ & $\mathrm{N} 3-\mathrm{O} 8$ & $1.222(2)$ \\
$\mathrm{C} 9-\mathrm{C} 8$ & $1.385(3)$ & $\mathrm{C} 4-\mathrm{C} 5$ & $1.373(3)$ \\
$\mathrm{O} 9-\mathrm{N} 3$ & $1.227(2)$ & $\mathrm{S} 2-\mathrm{C} 25$ & $1.813(2)$
\end{tabular}




$$
\begin{array}{ll|ll}
\mathrm{N} 2-\mathrm{O} 6 & 1.224(2) & \mathrm{C} 2-\mathrm{C} 3 & 1.385(2) \\
\mathrm{N} 2-\mathrm{O} 7 & 1.228(2) & \mathrm{C} 2-\mathrm{N} 3 & 1.479(2) \\
\mathrm{N} 2-\mathrm{C} 4 & 1.467(2) & &
\end{array}
$$

Table S39. Selected bond angles $\left(^{\circ}\right)$ of compound $7 \mathbf{b}$.

\begin{tabular}{|c|c|c|c|}
\hline $\mathrm{O} 2-\mathrm{S} 1-\mathrm{O} 1$ & $115.1(1)$ & $\mid \mathrm{C} 11-\mathrm{C} 10-\mathrm{C} 9$ & $120.3(2)$ \\
\hline $\mathrm{O} 2-\mathrm{S} 1-\mathrm{O} 3$ & $114.7(1)$ & $\mathrm{C} 11-\mathrm{C} 12-\mathrm{C} 7$ & $119.3(2)$ \\
\hline $\mathrm{O} 1-\mathrm{S} 1-\mathrm{O} 3$ & $113.4(1)$ & $\mathrm{C} 22-\mathrm{C} 23-\mathrm{C} 24$ & $120.1(2)$ \\
\hline $\mathrm{O} 2-\mathrm{S} 1-\mathrm{C} 1$ & $107.1(1)$ & $\mathrm{C} 16-\mathrm{C} 15-\mathrm{C} 14$ & $120.3(2)$ \\
\hline $\mathrm{O} 1-\mathrm{S} 1-\mathrm{C} 1$ & $102.6(1)$ & $\mathrm{C} 15-\mathrm{C} 14-\mathrm{C} 13$ & $119.2(2)$ \\
\hline $\mathrm{O} 3-\mathrm{S} 1-\mathrm{C} 1$ & $102.1(1)$ & $\mathrm{C} 14-\mathrm{C} 13-\mathrm{C} 18$ & $120.5(2)$ \\
\hline $\mathrm{C} 13-\mathrm{P} 1-\mathrm{C} 7$ & $110.7(1)$ & $\mathrm{C} 14-\mathrm{C} 13-\mathrm{P} 1$ & $120.0(1)$ \\
\hline $\mathrm{C} 13-\mathrm{P} 1-\mathrm{C} 19$ & $109.6(1)$ & $\mathrm{C} 18-\mathrm{C} 13-\mathrm{P} 1$ & $119.4(1)$ \\
\hline $\mathrm{C} 7-\mathrm{P} 1-\mathrm{C} 19$ & $109.6(1)$ & $\mathrm{F} 1-\mathrm{C} 25-\mathrm{S} 2$ & $111.3(1)$ \\
\hline $\mathrm{C} 13-\mathrm{P} 1-\mathrm{S} 2$ & $110.7(1)$ & $\mathrm{C} 24-\mathrm{C} 19-\mathrm{C} 20$ & $120.2(2)$ \\
\hline $\mathrm{C} 7-\mathrm{P} 1-\mathrm{S} 2$ & $102.6(1)$ & $\mathrm{C} 24-\mathrm{C} 19-\mathrm{P} 1$ & $119.8(2)$ \\
\hline $\mathrm{C} 19-\mathrm{P} 1-\mathrm{S} 2$ & $113.4(1)$ & $\mathrm{C} 20-\mathrm{C} 19-\mathrm{P} 1$ & $120.0(2)$ \\
\hline $\mathrm{O} 5-\mathrm{N} 1-\mathrm{O} 4$ & $125.4(2)$ & $\mathrm{C} 17-\mathrm{C} 18-\mathrm{C} 13$ & $119.4(2)$ \\
\hline $\mathrm{O} 5-\mathrm{N} 1-\mathrm{C} 6$ & $118.3(2)$ & $\mathrm{C} 23-\mathrm{C} 22-\mathrm{C} 21$ & $120.4(2)$ \\
\hline $\mathrm{O} 4-\mathrm{N} 1-\mathrm{C} 6$ & $116.2(2)$ & $\mathrm{C} 22-\mathrm{C} 21-\mathrm{C} 20$ & $120.4(2)$ \\
\hline $\mathrm{C} 2-\mathrm{C} 1-\mathrm{C} 6$ & $114.6(2)$ & $\mathrm{C} 18-\mathrm{C} 17-\mathrm{C} 16$ & $120.2(2)$ \\
\hline $\mathrm{C} 2-\mathrm{C} 1-\mathrm{S} 1$ & $123.0(1)$ & $\mathrm{C} 23-\mathrm{C} 24-\mathrm{C} 19$ & $119.6(2)$ \\
\hline $\mathrm{C} 6-\mathrm{C} 1-\mathrm{S} 1$ & $121.3(1)$ & $\mathrm{C} 21-\mathrm{C} 20-\mathrm{C} 19$ & $119.4(2)$ \\
\hline $\mathrm{C} 12-\mathrm{C} 11-\mathrm{C} 10$ & $120.5(2)$ & $\mathrm{C} 15-\mathrm{C} 16-\mathrm{C} 17$ & $120.5(2)$ \\
\hline $\mathrm{C} 10-\mathrm{C} 9-\mathrm{C} 8$ & $119.9(2)$ & $\mathrm{C} 5-\mathrm{C} 4-\mathrm{C} 3$ & $122.4(2)$ \\
\hline $\mathrm{O} 6-\mathrm{N} 2-\mathrm{O} 7$ & $125.2(2)$ & $\mathrm{C} 5-\mathrm{C} 4-\mathrm{N} 2$ & $118.8(2)$ \\
\hline $\mathrm{O} 6-\mathrm{N} 2-\mathrm{C} 4$ & $117.3(2)$ & $\mathrm{C} 3-\mathrm{C} 4-\mathrm{N} 2$ & $118.8(2)$ \\
\hline $\mathrm{O} 7-\mathrm{N} 2-\mathrm{C} 4$ & $117.5(2)$ & $\mathrm{C} 4-\mathrm{C} 5-\mathrm{C} 6$ & $117.7(2)$ \\
\hline $\mathrm{C} 25-\mathrm{S} 2-\mathrm{P} 1$ & $104.5(1)$ & $\mathrm{C} 5-\mathrm{C} 6-\mathrm{C} 1$ & $124.0(2)$ \\
\hline $\mathrm{C} 3-\mathrm{C} 2-\mathrm{C} 1$ & $124.0(2)$ & $\mathrm{C} 5-\mathrm{C} 6-\mathrm{N} 1$ & $115.5(2)$ \\
\hline $\mathrm{C} 3-\mathrm{C} 2-\mathrm{N} 3$ & $115.1(2)$ & $\mathrm{C} 1-\mathrm{C} 6-\mathrm{N} 1$ & $120.5(2)$ \\
\hline $\mathrm{C} 1-\mathrm{C} 2-\mathrm{N} 3$ & $121.0(2)$ & $\mathrm{C} 8-\mathrm{C} 7-\mathrm{C} 12$ & $120.1(2)$ \\
\hline $\mathrm{C} 4-\mathrm{C} 3-\mathrm{C} 2$ & $117.3(2)$ & $\mathrm{C} 8-\mathrm{C} 7-\mathrm{P} 1$ & $120.7(2)$ \\
\hline $\mathrm{O} 8-\mathrm{N} 3-\mathrm{O} 9$ & $125.2(2)$ & $\mathrm{C} 12-\mathrm{C} 7-\mathrm{P} 1$ & $119.2(2)$ \\
\hline $\mathrm{O} 8-\mathrm{N} 3-\mathrm{C} 2$ & $117.3(2)$ & $\mathrm{C} 9-\mathrm{C} 8-\mathrm{C} 7$ & $119.8(2)$ \\
\hline $\mathrm{O} 9-\mathrm{N} 3-\mathrm{C} 2$ & $117.5(2)$ & & \\
\hline
\end{tabular}

Table S40. Selected torsion angles $\left(^{\circ}\right)$ of compound $\mathbf{7 b}$. 


\begin{tabular}{|c|c|c|c|}
\hline $\mathrm{O} 2-\mathrm{S} 1-\mathrm{C} 1-\mathrm{C} 2$ & $94.3(2)$ & $\mathrm{P} 1-\mathrm{C} 7-\mathrm{C} 12$ & $52.1(2)$ \\
\hline $\mathrm{O} 1-\mathrm{S} 1-\mathrm{C} 1-\mathrm{C} 2$ & $-144.3(2)$ & $\mathrm{C} 10-\mathrm{C} 9-\mathrm{C} 8-\mathrm{C} 7$ & $-0.6(3)$ \\
\hline $3-\mathrm{S} 1-\mathrm{C} 1-\mathrm{C} 2$ & $-26.6(2)$ & $\mathrm{C} 12-\mathrm{C} 7-\mathrm{C} 8-\mathrm{C} 9$ & $0.2(3)$ \\
\hline $\mathrm{O} 2-\mathrm{S} 1-\mathrm{C} 1-\mathrm{C} 6$ & $-98.3(2)$ & $\mathrm{P} 1-\mathrm{C} 7-\mathrm{C} 8-\mathrm{C} 9$ & $-179.2(2)$ \\
\hline $\mathrm{O} 1-\mathrm{S} 1-\mathrm{C} 1-\mathrm{C} 6$ & $23.2(2)$ & $\mathrm{C} 12-\mathrm{C} 11-\mathrm{C} 10-\mathrm{C} 9$ & $0.9(3)$ \\
\hline $\mathrm{O} 3-\mathrm{S} 1-\mathrm{C} 1-\mathrm{C} 6$ & $140.8(2)$ & $\mathrm{C} 8-\mathrm{C} 9-\mathrm{C} 10-\mathrm{C} 11$ & $0.0(3)$ \\
\hline $\mathrm{C} 6-\mathrm{C} 1-\mathrm{C} 2-\mathrm{C} 3$ & $-2.4(3)$ & $\mathrm{C} 10-\mathrm{C} 11-\mathrm{C} 12-\mathrm{C} 7$ & $-1.3(3)$ \\
\hline $\mathrm{S} 1-\mathrm{C} 1-\mathrm{C} 2-\mathrm{C} 3$ & 2) & $\mathrm{C} 8-\mathrm{C} 7-$ & (3) \\
\hline $\mathrm{C} 6-\mathrm{C} 1-\mathrm{C} 2-\mathrm{N} 3$ & $177.4(2)$ & $\mathrm{P} 1-\mathrm{C} 7-\mathrm{C} 12-\mathrm{C} 11$ & $-179.9(2)$ \\
\hline $\mathrm{S} 1-\mathrm{C} 1-\mathrm{C} 2-\mathrm{N} 3$ & $-14.3(2)$ & $\mathrm{C} 16-\mathrm{C} 15-\mathrm{C} 14-\mathrm{C} 13$ & $-1.2(3)$ \\
\hline $1-\mathrm{C} 2-\mathrm{C} 3-\mathrm{C} 4$ & $1.1(3)$ & $\mathrm{C} 15-\mathrm{C} 14-\mathrm{C} 13-\mathrm{C} 18$ & $0.2(3)$ \\
\hline $\mathrm{N} 3-\mathrm{C} 2-\mathrm{C} 3-\mathrm{C} 4$ & $-178.8(2)$ & $\mathrm{C} 15-\mathrm{C} 14-\mathrm{C} 13-\mathrm{P} 1$ & $-175.9(2)$ \\
\hline $\mathrm{C} 3-\mathrm{C} 2-\mathrm{N} 3-\mathrm{O} 8$ & $-46.5(2)$ & $\mathrm{C} 7-\mathrm{P} 1-\mathrm{C} 13-\mathrm{C} 14$ & $104.1(2)$ \\
\hline $\mathrm{C} 1-\mathrm{C} 2-\mathrm{N} 3-\mathrm{O} 8$ & $133.7(2)$ & $\mathrm{C} 19-\mathrm{P} 1-\mathrm{C} 13-\mathrm{C} 14$ & $-16.9(2)$ \\
\hline $3-\mathrm{C} 2-\mathrm{N} 3-\mathrm{O} 9$ & $130.3(2)$ & $\mathrm{S} 2-\mathrm{P} 1-\mathrm{C} 13-\mathrm{C} 14$ & $-142.8(2)$ \\
\hline $\mathrm{C} 1-\mathrm{C} 2-\mathrm{N} 3-\mathrm{O} 9$ & $-49.6(2)$ & $\mathrm{C} 7-\mathrm{P} 1-\mathrm{C} 13-\mathrm{C} 18$ & $-72.1(2)$ \\
\hline $\mathrm{C} 2-\mathrm{C} 3-\mathrm{C} 4-\mathrm{C} 5$ & $0.0(3)$ & $\mathrm{C} 19-\mathrm{P} 1-\mathrm{C} 13-\mathrm{C} 18$ & $166.9(2)$ \\
\hline $\mathrm{C} 2-\mathrm{C} 3-\mathrm{C} 4-\mathrm{N} 2$ & $-179.5(2)$ & $\mathrm{S} 2-\mathrm{P} 1-\mathrm{C} 13-\mathrm{C} 18$ & $41.0(2)$ \\
\hline $\mathrm{O} 6-\mathrm{N} 2-\mathrm{C} 4-\mathrm{C} 5$ & $16.4(3)$ & $\mathrm{P} 1-\mathrm{S} 2-\mathrm{C} 25-\mathrm{F} 1$ & $88.5(1)$ \\
\hline $\mathrm{O} 7-\mathrm{N} 2-\mathrm{C} 4-\mathrm{C} 5$ & $-163.3(2)$ & $\mathrm{C} 13-\mathrm{P} 1-\mathrm{C} 19-\mathrm{C} 24$ & $103.6(2)$ \\
\hline $\mathrm{O} 6-\mathrm{N} 2-\mathrm{C} 4-\mathrm{C} 3$ & $-164.1(2)$ & $\mathrm{C} 7-\mathrm{P} 1-\mathrm{C} 19-\mathrm{C} 24$ & $-18.2(2)$ \\
\hline $\mathrm{O} 7-\mathrm{N} 2-\mathrm{C} 4-\mathrm{C} 3$ & $16.3(3)$ & $\mathrm{S} 2-\mathrm{P} 1-\mathrm{C} 19-\mathrm{C} 24$ & $-132.2(2)$ \\
\hline $\mathrm{C} 3-\mathrm{C} 4-\mathrm{C} 5-\mathrm{C} 6$ & $0.5(3)$ & $\mathrm{C} 13-\mathrm{P} 1-\mathrm{C} 19-\mathrm{C} 20$ & $-73.3(2)$ \\
\hline $\mathrm{N} 2-\mathrm{C} 4-\mathrm{C} 5-\mathrm{C} 6$ & $180.0(2)$ & $\mathrm{C} 7-\mathrm{P} 1-\mathrm{C} 19-\mathrm{C} 20$ & $165.0(2)$ \\
\hline $\mathrm{C} 4-\mathrm{C} 5-\mathrm{C} 6-\mathrm{C} 1$ & $-2.0(3)$ & $\mathrm{S} 2-\mathrm{P} 1-\mathrm{C} 19-\mathrm{C} 20$ & $51.0(2)$ \\
\hline $\mathrm{C} 4-\mathrm{C} 5-\mathrm{C} 6-\mathrm{N} 1$ & $175.5(2)$ & $\mathrm{C} 14-\mathrm{C} 13-\mathrm{C} 18-\mathrm{C} 17$ & $1.1(3)$ \\
\hline $\mathrm{C} 2-\mathrm{C} 1-\mathrm{C} 6-\mathrm{C} 5$ & $2.9(3)$ & $\mathrm{P} 1-\mathrm{C} 13-\mathrm{C} 18-\mathrm{C} 17$ & $177.3(2)$ \\
\hline $\mathrm{S} 1-\mathrm{C} 1-\mathrm{C} 6-\mathrm{C} 5$ & $-165.6(2)$ & $\mathrm{C} 24-\mathrm{C} 23-\mathrm{C} 22-\mathrm{C} 21$ & $0.4(3)$ \\
\hline $\mathrm{C} 2-\mathrm{C} 1-\mathrm{C} 6-\mathrm{N} 1$ & $-174.5(2)$ & $\mathrm{C} 23-\mathrm{C} 22-\mathrm{C} 21-\mathrm{C} 20$ & $-0.2(3)$ \\
\hline $\mathrm{S} 1-\mathrm{C} 1-\mathrm{C} 6-\mathrm{N} 1$ & $17.1(2)$ & $\mathrm{C} 13-\mathrm{C} 18-\mathrm{C} 17-\mathrm{C} 16$ & $-1.5(3)$ \\
\hline $\mathrm{O} 5-\mathrm{N} 1-\mathrm{C} 6-\mathrm{C} 5$ & $58.4(2)$ & $\mathrm{C} 22-\mathrm{C} 23-\mathrm{C} 24-\mathrm{C} 19$ & $-0.1(3)$ \\
\hline $\mathrm{O} 4-\mathrm{N} 1-\mathrm{C} 6-\mathrm{C} 5$ & $-117.5(2)$ & $\mathrm{C} 20-\mathrm{C} 19-\mathrm{C} 24-\mathrm{C} 23$ & $-0.3(3)$ \\
\hline $\mathrm{O} 5-\mathrm{N} 1-\mathrm{C} 6-\mathrm{C} 1$ & $-124.1(2)$ & $\mathrm{P} 1-\mathrm{C} 19-\mathrm{C} 24-\mathrm{C} 23$ & $-177.2(2)$ \\
\hline $\mathrm{O} 4-\mathrm{N} 1-\mathrm{C} 6-\mathrm{C} 1$ & $60.0(2)$ & $\mathrm{C} 22-\mathrm{C} 21-\mathrm{C} 20-\mathrm{C} 19$ & $-0.3(3)$ \\
\hline $\mathrm{C} 13-\mathrm{P} 1-\mathrm{C} 7-\mathrm{C} 8$ & $-10.4(2)$ & $\mathrm{C} 24-\mathrm{C} 19-\mathrm{C} 20-\mathrm{C} 21$ & $0.5(3)$ \\
\hline $\mathrm{C} 19-\mathrm{P} 1-\mathrm{C} 7-\mathrm{C} 8$ & $110.6(2)$ & $\mathrm{P} 1-\mathrm{C} 19-\mathrm{C} 20-\mathrm{C} 21$ & $177.4(2)$ \\
\hline $\mathrm{S} 2-\mathrm{P} 1-\mathrm{C} 7-\mathrm{C} 8$ & $-128.6(2)$ & $\mathrm{C} 14-\mathrm{C} 15-\mathrm{C} 16-\mathrm{C} 17$ & $0.8(3)$ \\
\hline $\mathrm{C} 13-\mathrm{P} 1-\mathrm{C} 7-\mathrm{C} 12$ & $170.3(2)$ & $\mathrm{C} 18-\mathrm{C} 17-\mathrm{C} 16-\mathrm{C} 15$ & $0.6(3)$ \\
\hline $\mathrm{C} 19-\mathrm{P} 1-\mathrm{C} 7-\mathrm{C} 12$ & $-68.8(2)$ & & \\
\hline
\end{tabular}


2.11 Compound 9

Table S41. Details for X-ray data collection and structure refinement for compound 9.

\begin{tabular}{ll}
\hline & 9 \\
\hline CCDC & 2025113 \\
Empirical formula & $\mathrm{C}_{18} \mathrm{H}_{12} \mathrm{FN}_{5} \mathrm{O}_{10} \mathrm{~S}$ \\
& $\mathrm{~S} 66$
\end{tabular}




\begin{tabular}{|c|c|}
\hline Formula mass & 509.39 \\
\hline $\mathrm{T}[\mathrm{K}]$ & $143(2)$ \\
\hline Crystal size $[\mathrm{mm}]$ & $0.10 \times 0.05 \times 0.05$ \\
\hline Crystal description & colorless needle \\
\hline Crystal system & triclinic \\
\hline Space group & $P-1$ \\
\hline 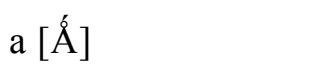 & $5.5545(4)$ \\
\hline $\mathrm{b}[\AA ̊ 丿]$ & $10.7059(9)$ \\
\hline $\mathrm{c}[\AA \hat{A}]$ & $18.2101(17)$ \\
\hline$\alpha\left[^{\circ}\right]$ & $81.755(7)$ \\
\hline$\beta\left[^{\circ}\right]$ & $83.317(7)$ \\
\hline$\gamma\left[^{\circ}\right]$ & $76.062(7)$ \\
\hline $\mathrm{V}\left[\AA^{3}\right]$ & $1036.35(15)$ \\
\hline Z & 2 \\
\hline$\rho_{\text {calcd. }}\left[\mathrm{g} \mathrm{cm}^{-3}\right]$ & 1.632 \\
\hline$\mu\left[\mathrm{mm}^{-1}\right]$ & 0.236 \\
\hline$F(000)$ & 520 \\
\hline$\Theta$ range $\left[{ }^{\circ}\right]$ & $4.18-25.24$ \\
\hline \multirow[t]{3}{*}{ Index ranges } & $-6 \leq h \leq 6$ \\
\hline & $-13 \leq k \leq 13$ \\
\hline & $-22 \leq l \leq 22$ \\
\hline Reflns. collected & 7821 \\
\hline Reflns. obsd. & 2148 \\
\hline Reflns. unique & $\begin{array}{l}4205 \\
\left(R_{\text {int }}=0.0668\right)\end{array}$ \\
\hline$R_{1}, w R_{2}(2 \sigma$ data $)$ & $0.0677,0.1051$ \\
\hline$R_{1}, w R_{2}($ all data $)$ & $0.1511,0.1368$ \\
\hline GOOF on $F^{2}$ & 0.979 \\
\hline Peak/hole [e $\AA^{-3}$ ] & $0.346 /-0.309$ \\
\hline
\end{tabular}



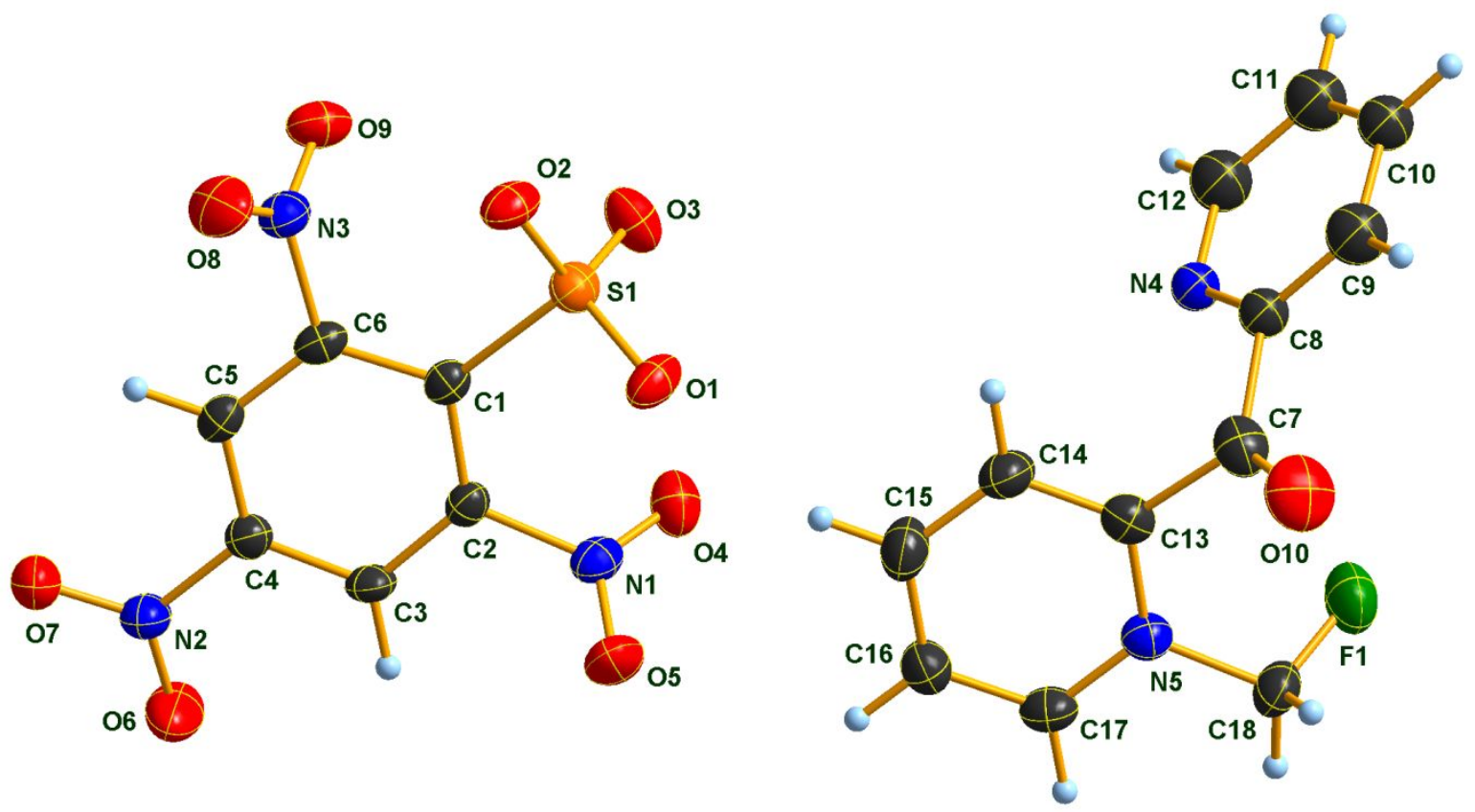

Figure S11. Molecular structure of compound 9 in the crystal. DIAMOND ${ }^{\mathrm{e})}$ representation; thermal ellipsoids are drawn at $50 \%$ probability level. The non-fluoromethylated pyridine ring is disordered over two positions; only the more populated position has been shown for clarity.

Table S42. Selected bond lengths $(\AA)$ of compound 9.

\begin{tabular}{ll|ll}
$\mathrm{S} 1-\mathrm{O} 3$ & $1.440(3)$ & $\mathrm{C} 9-\mathrm{C} 10$ & $1.380(13)$ \\
$\mathrm{S} 1-\mathrm{O} 2$ & $1.445(3)$ & $\mathrm{C} 10-\mathrm{C} 11$ & $1.374(13)$ \\
$\mathrm{S} 1-\mathrm{C} 1$ & $1.817(4)$ & $\mathrm{C} 11-\mathrm{C} 12$ & $1.366(14)$ \\
$\mathrm{N} 1-\mathrm{O} 5$ & $1.218(4)$ & $\mathrm{C} 12-\mathrm{N} 4$ & $1.326(11)$ \\
$\mathrm{N} 1-\mathrm{O} 4$ & $1.222(4)$ & $\mathrm{C} 17-\mathrm{C} 16$ & $1.370(5)$ \\
$\mathrm{N} 1-\mathrm{C} 2$ & $1.479(5)$ & $\mathrm{C} 16-\mathrm{C} 15$ & $1.375(6)$ \\
$\mathrm{C} 1-\mathrm{C} 2$ & $1.389(5)$ & $\mathrm{C} 15-\mathrm{C} 14$ & $1.373(6)$ \\
$\mathrm{C} 1-\mathrm{C} 6$ & $1.391(5)$ & $\mathrm{C} 14-\mathrm{C} 13$ & $1.362(5)$ \\
$\mathrm{F} 1-\mathrm{C} 18$ & $1.357(4)$ & $\mathrm{C} 4-\mathrm{C} 5$ & $1.364(5)$ \\
$\mathrm{N} 2-\mathrm{O} 7$ & $1.222(4)$ & $\mathrm{N} 5-\mathrm{C} 17$ & $1.340(5)$ \\
$\mathrm{N} 2-\mathrm{O} 6$ & $1.225(4)$ & $\mathrm{N} 5-\mathrm{C} 13$ & $1.358(5)$ \\
$\mathrm{N} 2-\mathrm{C} 4$ & $1.468(5)$ & $\mathrm{N} 5-\mathrm{C} 18$ & $1.480(4)$ \\
$\mathrm{C} 2-\mathrm{C} 3$ & $1.387(5)$ & $\mathrm{C} 5-\mathrm{C} 6$ & $1.384(5)$ \\
$\mathrm{N} 3-\mathrm{O} 8$ & $1.210(4)$ & $\mathrm{C} 7-\mathrm{O} 10$ & $1.210(5)$ \\
$\mathrm{N} 3-\mathrm{O} 9$ & $1.225(4)$ & $\mathrm{C} 7-\mathrm{C} 13$ & $1.525(6)$ \\
$\mathrm{N} 3-\mathrm{C} 6$ & $1.479(5)$ & $\mathrm{C} 7-\mathrm{C} 8$ & $1.594(12)$ \\
$\mathrm{C} 3-\mathrm{C} 4$ & $1.373(5)$ & $\mathrm{C} 8-\mathrm{N} 4$ & $1.290(14)$
\end{tabular}


Table S43. Selected bond angles $\left(^{\circ}\right)$ of compound 9.

\begin{tabular}{|c|c|c|c|}
\hline $\mathrm{O} 1-\mathrm{S} 1-\mathrm{O} 3$ & $115.0(2)$ & C11 - C10-C9 & $115.5(9)$ \\
\hline $\mathrm{O} 1-\mathrm{S} 1-\mathrm{O} 2$ & $113.3(2)$ & $\mathrm{C} 12-\mathrm{C} 11-\mathrm{C} 10$ & $121.0(9)$ \\
\hline $\mathrm{O} 3-\mathrm{S} 1-\mathrm{O} 2$ & $113.7(2)$ & $\mathrm{N} 4-\mathrm{C} 12-\mathrm{C} 11$ & $121.7(9)$ \\
\hline $\mathrm{O} 1-\mathrm{S} 1-\mathrm{C} 1$ & $102.9(2)$ & $\mathrm{C} 8-\mathrm{N} 4-\mathrm{C} 12$ & $118.3(8)$ \\
\hline $\mathrm{O} 3-\mathrm{S} 1-\mathrm{C} 1$ & $106.2(2)$ & $\mathrm{N} 5-\mathrm{C} 17-\mathrm{C} 16$ & $120.5(4)$ \\
\hline $\mathrm{O} 2-\mathrm{S} 1-\mathrm{C} 1$ & $104.2(2)$ & $\mathrm{C} 17-\mathrm{C} 16-\mathrm{C} 15$ & $119.1(4)$ \\
\hline $\mathrm{O} 5-\mathrm{N} 1-\mathrm{O} 4$ & $125.3(3)$ & $\mathrm{C} 14-\mathrm{C} 15-\mathrm{C} 16$ & $119.4(4)$ \\
\hline $\mathrm{O} 5-\mathrm{N} 1-\mathrm{C} 2$ & $117.2(3)$ & $\mathrm{C} 13-\mathrm{C} 14-\mathrm{C} 15$ & $120.5(4)$ \\
\hline $\mathrm{O} 4-\mathrm{N} 1-\mathrm{C} 2$ & $117.5(3)$ & N5 $-\mathrm{C} 13-\mathrm{C} 14$ & $119.0(4)$ \\
\hline $\mathrm{C} 2-\mathrm{C} 1-\mathrm{C} 6$ & $114.8(3)$ & $\mathrm{N} 5-\mathrm{C} 13-\mathrm{C} 7$ & $119.1(3)$ \\
\hline $\mathrm{C} 2-\mathrm{C} 1-\mathrm{S} 1$ & $122.4(3)$ & $\mathrm{C} 14-\mathrm{C} 13-\mathrm{C} 7$ & $121.5(4)$ \\
\hline $\mathrm{C} 6-\mathrm{C} 1-\mathrm{S} 1$ & $122.5(3)$ & $\mathrm{F} 1-\mathrm{C} 18-\mathrm{N} 5$ & $107.8(3)$ \\
\hline $\mathrm{O} 7-\mathrm{N} 2-\mathrm{O} 6$ & $123.9(3)$ & $\mathrm{C} 13-\mathrm{C} 7-\mathrm{C} 8$ & $112.6(5)$ \\
\hline $\mathrm{O} 7-\mathrm{N} 2-\mathrm{C} 4$ & $118.0(3)$ & $\mathrm{N} 4-\mathrm{C} 8-\mathrm{C} 9$ & $123.4(10)$ \\
\hline $\mathrm{O} 6-\mathrm{N} 2-\mathrm{C} 4$ & $118.0(3)$ & $\mathrm{N} 4-\mathrm{C} 8-\mathrm{C} 7$ & $118.1(8)$ \\
\hline $\mathrm{C} 3-\mathrm{C} 2-\mathrm{C} 1$ & $123.8(3)$ & $\mathrm{C} 9-\mathrm{C} 8-\mathrm{C} 7$ & $118.5(9)$ \\
\hline $\mathrm{C} 3-\mathrm{C} 2-\mathrm{N} 1$ & $114.8(3)$ & $\mathrm{C} 8-\mathrm{C} 9-\mathrm{C} 10$ & $119.9(10)$ \\
\hline $\mathrm{C} 1-\mathrm{C} 2-\mathrm{N} 1$ & $121.3(3)$ & $\mathrm{C} 17-\mathrm{N} 5-\mathrm{C} 13$ & $121.4(3)$ \\
\hline $\mathrm{O} 8-\mathrm{N} 3-\mathrm{O} 9$ & $125.6(3)$ & $\mathrm{C} 17-\mathrm{N} 5-\mathrm{C} 18$ & $118.0(3)$ \\
\hline $\mathrm{O} 8-\mathrm{N} 3-\mathrm{C} 6$ & $118.0(3)$ & $\mathrm{C} 13-\mathrm{N} 5-\mathrm{C} 18$ & $120.7(3)$ \\
\hline $\mathrm{O} 9-\mathrm{N} 3-\mathrm{C} 6$ & $116.4(3)$ & $\mathrm{C} 4-\mathrm{C} 5-\mathrm{C} 6$ & $117.9(3)$ \\
\hline $\mathrm{C} 4-\mathrm{C} 3-\mathrm{C} 2$ & $117.5(4)$ & $\mathrm{C} 5-\mathrm{C} 6-\mathrm{C} 1$ & $123.6(4)$ \\
\hline $\mathrm{C} 5-\mathrm{C} 4-\mathrm{C} 3$ & $122.3(4)$ & $\mathrm{C} 5-\mathrm{C} 6-\mathrm{N} 3$ & $115.1(3)$ \\
\hline $\mathrm{C} 5-\mathrm{C} 4-\mathrm{N} 2$ & $119.3(3)$ & $\mathrm{C} 1-\mathrm{C} 6-\mathrm{N} 3$ & $121.3(3)$ \\
\hline $\mathrm{C} 3-\mathrm{C} 4-\mathrm{N} 2$ & $118.5(3)$ & $\mathrm{O} 10-\mathrm{C} 7-\mathrm{C} 13$ & $118.5(4)$ \\
\hline $\mathrm{O} 10-\mathrm{C} 7-\mathrm{C} 8$ & $128.9(6)$ & & \\
\hline
\end{tabular}

Table S44. Selected torsion angles $\left(^{\circ}\right)$ of compound 9.

\begin{tabular}{ll|ll}
$\mathrm{O} 1-\mathrm{S} 1-\mathrm{C} 1-\mathrm{C} 2$ & $-33.1(4)$ & $\mathrm{C} 7-\mathrm{C} 8-\mathrm{C} 9-\mathrm{C} 10$ & $174.7(8)$ \\
$\mathrm{O} 3-\mathrm{S} 1-\mathrm{C} 1-\mathrm{C} 2$ & $88.1(3)$ & $\mathrm{C} 8-\mathrm{C} 9-\mathrm{C} 10-\mathrm{C} 11$ & $0.4(14)$ \\
$\mathrm{O} 2-\mathrm{S} 1-\mathrm{C} 1-\mathrm{C} 2$ & $-151.6(3)$ & $\mathrm{C} 9-\mathrm{C} 10-\mathrm{C} 11-\mathrm{C} 12$ & $2.3(13)$ \\
$\mathrm{O} 1-\mathrm{S} 1-\mathrm{C} 1-\mathrm{C} 6$ & $140.9(3)$ & $\mathrm{C} 10-\mathrm{C} 11-\mathrm{C} 12-\mathrm{N} 4$ & $-2.2(14)$ \\
$\mathrm{O} 3-\mathrm{S} 1-\mathrm{C} 1-\mathrm{C} 6$ & $-98.0(3)$ & $\mathrm{C} 9-\mathrm{C} 8-\mathrm{N} 4-\mathrm{C} 12$ & $3.8(16)$ \\
$\mathrm{O} 2-\mathrm{S} 1-\mathrm{C} 1-\mathrm{C} 6$ & $22.4(4)$ & $\mathrm{C} 7-\mathrm{C} 8-\mathrm{N} 4-\mathrm{C} 12$ & $-174.5(7)$ \\
$\mathrm{C} 6-\mathrm{C} 1-\mathrm{C} 2-\mathrm{C} 3$ & $-0.6(6)$ & $\mathrm{C} 11-\mathrm{C} 12-\mathrm{N} 4-\mathrm{C} 8$ & $-0.9(14)$ \\
$\mathrm{S} 1-\mathrm{C} 1-\mathrm{C} 2-\mathrm{C} 3$ & $173.8(3)$ & $\mathrm{C} 13-\mathrm{N} 5-\mathrm{C} 17-\mathrm{C} 16$ & $-0.5(6)$ \\
$\mathrm{C} 6-\mathrm{C} 1-\mathrm{C} 2-\mathrm{N} 1$ & $179.7(3)$ & $\mathrm{C} 18-\mathrm{N} 5-\mathrm{C} 17-\mathrm{C} 16$ & $178.1(3)$
\end{tabular}




\begin{tabular}{ll|ll}
$\mathrm{S} 1-\mathrm{C} 1-\mathrm{C} 2-\mathrm{N} 1$ & $-5.9(5)$ & $\mathrm{N} 5-\mathrm{C} 17-\mathrm{C} 16-\mathrm{C} 15$ & $-0.1(6)$ \\
$\mathrm{O} 5-\mathrm{N} 1-\mathrm{C} 2-\mathrm{C} 3$ & $-56.3(5)$ & $\mathrm{C} 17-\mathrm{C} 16-\mathrm{C} 15-\mathrm{C} 14$ & $1.4(7)$ \\
$\mathrm{O} 4-\mathrm{N} 1-\mathrm{C} 2-\mathrm{C} 3$ & $120.9(4)$ & $\mathrm{C} 16-\mathrm{C} 15-\mathrm{C} 14-\mathrm{C} 13$ & $-2.1(7)$ \\
$\mathrm{O} 5-\mathrm{N} 1-\mathrm{C} 2-\mathrm{C} 1$ & $123.4(4)$ & $\mathrm{C} 17-\mathrm{N} 5-\mathrm{C} 13-\mathrm{C} 14$ & $-0.3(6)$ \\
$\mathrm{O} 4-\mathrm{N} 1-\mathrm{C} 2-\mathrm{C} 1$ & $-59.4(5)$ & $\mathrm{C} 18-\mathrm{N} 5-\mathrm{C} 13-\mathrm{C} 14$ & $-178.8(4)$ \\
$\mathrm{C} 1-\mathrm{C} 2-\mathrm{C} 3-\mathrm{C} 4$ & $1.1(6)$ & $\mathrm{C} 17-\mathrm{N} 5-\mathrm{C} 13-\mathrm{C} 7$ & $172.9(4)$ \\
$\mathrm{N} 1-\mathrm{C} 2-\mathrm{C} 3-\mathrm{C} 4$ & $-179.1(3)$ & $\mathrm{C} 18-\mathrm{N} 5-\mathrm{C} 13-\mathrm{C} 7$ & $-5.7(6)$ \\
$\mathrm{C} 2-\mathrm{C} 3-\mathrm{C} 4-\mathrm{C} 5$ & $-2.2(6)$ & $\mathrm{C} 15-\mathrm{C} 14-\mathrm{C} 13-\mathrm{N} 5$ & $1.6(6)$ \\
$\mathrm{C} 2-\mathrm{C} 3-\mathrm{C} 4-\mathrm{N} 2$ & $177.1(4)$ & $\mathrm{C} 15-\mathrm{C} 14-\mathrm{C} 13-\mathrm{C} 7$ & $-171.4(4)$ \\
$\mathrm{O} 7-\mathrm{N} 2-\mathrm{C} 4-\mathrm{C} 5$ & $-10.8(5)$ & $\mathrm{O} 10-\mathrm{C} 7-\mathrm{C} 13-\mathrm{N} 5$ & $-60.2(6)$ \\
$\mathrm{O} 6-\mathrm{N} 2-\mathrm{C} 4-\mathrm{C} 5$ & $167.8(4)$ & $\mathrm{C} 8-\mathrm{C} 7-\mathrm{C} 13-\mathrm{N} 5$ & $120.8(5)$ \\
$\mathrm{O} 7-\mathrm{N} 2-\mathrm{C} 4-\mathrm{C} 3$ & $169.9(3)$ & $\mathrm{O} 10-\mathrm{C} 7-\mathrm{C} 13-\mathrm{C} 14$ & $112.8(5)$ \\
$\mathrm{O} 6-\mathrm{N} 2-\mathrm{C} 4-\mathrm{C} 3$ & $-11.5(5)$ & $\mathrm{C} 8-\mathrm{C} 7-\mathrm{C} 13-\mathrm{C} 14$ & $-66.2(6)$ \\
$\mathrm{C} 3-\mathrm{C} 4-\mathrm{C} 5-\mathrm{C} 6$ & $2.8(6)$ & $\mathrm{C} 17-\mathrm{N} 5-\mathrm{C} 18-\mathrm{F} 1$ & $133.5(4)$ \\
$\mathrm{N} 2-\mathrm{C} 4-\mathrm{C} 5-\mathrm{C} 6$ & $-176.5(3)$ & $\mathrm{C} 13-\mathrm{N} 5-\mathrm{C} 18-\mathrm{F} 1$ & $-47.9(5)$ \\
$\mathrm{C} 4-\mathrm{C} 5-\mathrm{C} 6-\mathrm{C} 1$ & $-2.3(6)$ & $\mathrm{O} 8-\mathrm{N} 3-\mathrm{C} 6-\mathrm{C} 1$ & $-113.8(4)$ \\
$\mathrm{C} 4-\mathrm{C} 5-\mathrm{C} 6-\mathrm{N} 3$ & $178.0(3)$ & $\mathrm{O} 9-\mathrm{N} 3-\mathrm{C} 6-\mathrm{C} 1$ & $68.1(5)$ \\
$\mathrm{C} 2-\mathrm{C} 1-\mathrm{C} 6-\mathrm{C} 5$ & $1.2(6)$ & $\mathrm{O} 10-\mathrm{C} 7-\mathrm{C} 8-\mathrm{N} 4$ & $174.6(7)$ \\
$\mathrm{S} 1-\mathrm{C} 1-\mathrm{C} 6-\mathrm{C} 5$ & $-173.2(3)$ & $\mathrm{C} 13-\mathrm{C} 7-\mathrm{C} 8-\mathrm{N} 4$ & $-6.5(10)$ \\
$\mathrm{C} 2-\mathrm{C} 1-\mathrm{C} 6-\mathrm{N} 3$ & $-179.0(3)$ & $\mathrm{O} 10-\mathrm{C} 7-\mathrm{C} 8-\mathrm{C} 9$ & $-3.8(12)$ \\
$\mathrm{S} 1-\mathrm{C} 1-\mathrm{C} 6-\mathrm{N} 3$ & $6.6(5)$ & $\mathrm{C} 13-\mathrm{C} 7-\mathrm{C} 8-\mathrm{C} 9$ & $175.1(8)$ \\
$\mathrm{O} 8-\mathrm{N} 3-\mathrm{C} 6-\mathrm{C} 5$ & $66.0(5)$ & $\mathrm{N} 4-\mathrm{C} 8-\mathrm{C} 9-\mathrm{C} 10$ & $-3.6(17)$ \\
$\mathrm{O} 9-\mathrm{N} 3-\mathrm{C} 6-\mathrm{C} 5$ & $-112.1(4)$ &
\end{tabular}

\section{References}

a) Program package 'CrysAlisPro 1.171.39.46e (Rigaku OD, 2018)'.

b) Sheldrick, G. M. (1997) SHELXS-97: Program for Crystal Structure Solution, University of Göttingen, Germany.

c) Sheldrick, G. M. (1997) SHELXL-97: Program for the Refinement of Crystal Structures, University of Göttingen, Germany.

d) Spek, A. L. (1999) PLATON: A Multipurpose Crystallographic Tool, Utrecht University, Utrecht, The Netherlands.

e) DIAMOND, Crystal Impact GbR., Version 3.2i.

3 DSC of compound 4 


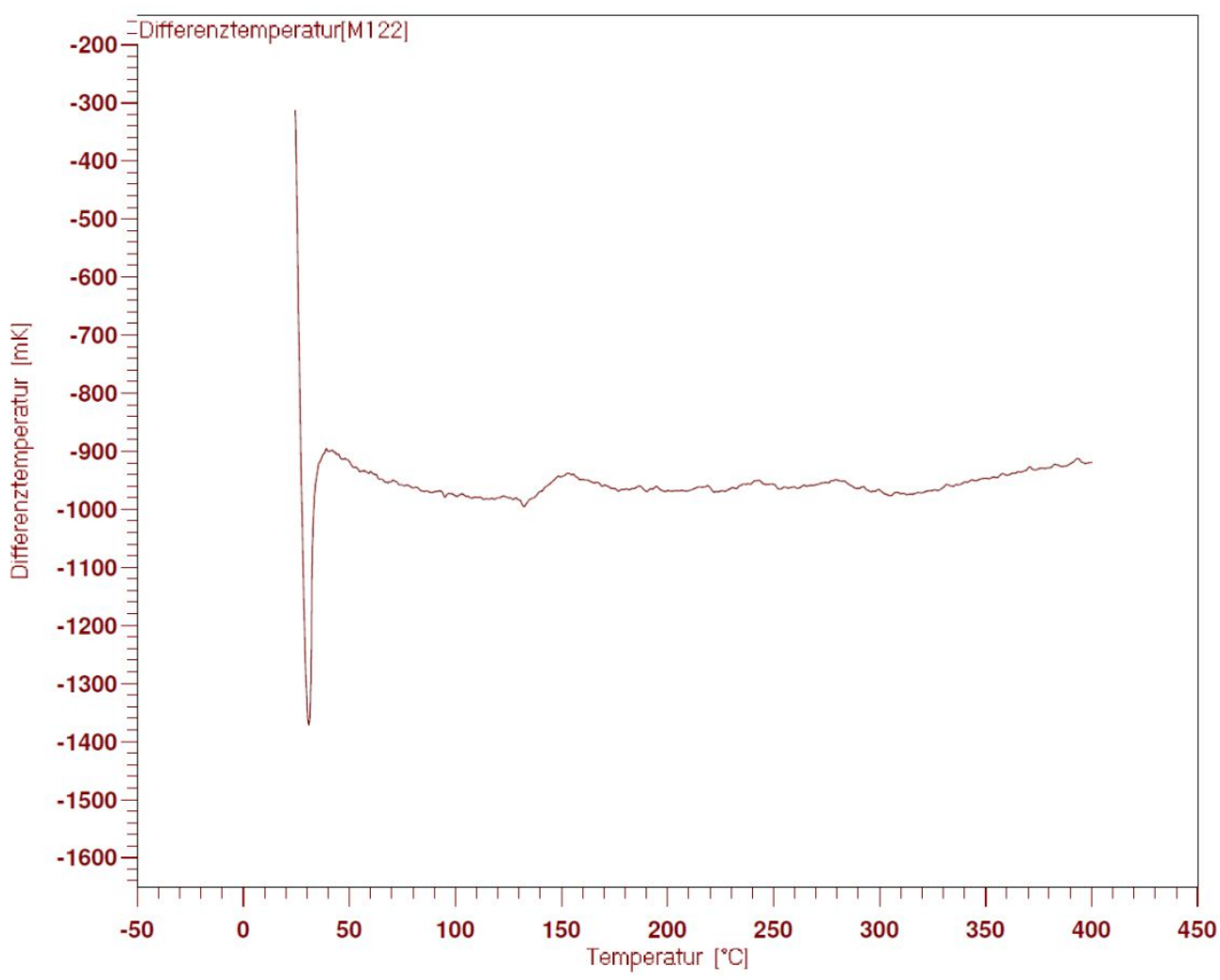

